(c) 2005 International Press

Adv. Theor. Math. Phys. 9 (2005) 931-998

\title{
On supertwistors, the
}

\section{Penrose-Ward transform and}

\section{$\mathcal{N}=4$ super-Yang-Mills theory}

\author{
Alexander D. Popov* and Christian Sämann \\ Institut für Theoretische Physik, Universität Hannover, Appelstraße 2, \\ 30167 Hannover, Germany \\ popov, saemann@itp.uni-hannover.de
}

\begin{abstract}
It was recently shown by Witten that B-type open topological string theory with the supertwistor space $\mathbb{C} P^{3 \mid 4}$ as a target space is equivalent to holomorphic Chern-Simons (hCS) theory on the same space. This hCS theory in turn is equivalent to self-dual $\mathcal{N}=4$ super-Yang-Mills (SYM) theory in four dimensions. We review the supertwistor description of self-dual and anti-self-dual $\mathcal{N}$-extended SYM theory as the integrability of SYM fields on complex $(2 \mid \mathcal{N})$-dimensional superplanes and demonstrate the equivalence of this description to Witten's formulation. The equivalence of the field equations of hCS theory on an open subset of $\mathbb{C} P^{3 \mid \mathcal{N}}$ to the field equations of self-dual $\mathcal{N}$-extended SYM theory in four dimensions is made explicit. Furthermore, we extend the picture to the full $\mathcal{N}=4$ SYM theory and, by using the known supertwistor description of this case, we show that the corresponding constraint equations are (gauge) equivalent to the field equations of hCS theory on a quadric in $\mathbb{C} P^{3 \mid 3} \times \mathbb{C} P^{3 \mid 3}$.
\end{abstract}

e-print archive: http://lanl.arXiv.org/abs/arXiv:hep-th/0405123v5 23 Jan 2006

* On leave from Bogoliubov Laboratory of Theoretical Physics, JINR, Dubna, Russia. 
1. Introduction 932

2. Twistor geometry 935

3. Twistor description of self-dual Yang-Mills fields 940

4. Supertwistor geometry 945

5. Supertwistor description of self-dual $\mathcal{N}$-extended SYM theory 950

6. Dual supertwistors and $\mathcal{N}$-extended anti-self-duality 958

7. A quadric in a product of supertwistor spaces 963

$\begin{array}{ll}\text { 8. hCS theory on the quadric } & 967\end{array}$

9. Supertwistors and the full $\mathcal{N}=4$ SYM theory 971

10. Reality conditions on the quadric 974

$\begin{array}{ll}\text { 11. Conclusions } & 979\end{array}$

$\begin{array}{ll}\text { Acknowledgments } & 981\end{array}$

Appendix A. Dictionary: homogeneous $\longleftrightarrow$ inhomogeneous coordinates 981

Appendix B. Some mathematical definitions 983

Appendix C. The twistor geometry in the Kleinian
case $\varepsilon=+1$

Appendix D. Comments on hCS theory in the Kleinian
case $\varepsilon=+1$

$\begin{array}{ll}\text { References } & 994\end{array}$

\section{Introduction}

Let $\mathcal{Z}$ be a complex three-dimensional Calabi-Yau (CY) manifold, $E$ a rank $n$ complex vector bundle over $\mathcal{Z}$ and $A$ a connection one-form on $E$. Consider 
the action [1]

$$
S_{\mathrm{hCS}}=\int_{\mathcal{Z}} \Omega_{0} \wedge \operatorname{tr}\left(A^{0,1} \wedge \bar{\partial} A^{0,1}+\frac{2}{3} A^{0,1} \wedge A^{0,1} \wedge A^{0,1}\right)
$$

where $\Omega_{0}$ is the nowhere vanishing holomorphic (3,0)-form on $\mathcal{Z}$ and $A^{0,1}$ is the $(0,1)$-component of the connection one-form $A$. Witten has obtained equation (1.1) as the full target space action of the open topological B-model on a complex three-dimensional target space, on which the CY restriction arises from $N=2$ supersymmetry of the corresponding topological sigma model and an anomaly cancellation condition. This holomorphic ChernSimons (hCS) theory (1.1) describes inequivalent complex structures on the bundle $E \rightarrow \mathcal{Z}$.

In a beautiful recent paper [2], Witten observed that the above-mentioned severe CY restriction can be relaxed by considering a topological B-model whose target space is a CY supermanifold. ${ }^{1}$ Here, the fermionic dimensions will also make a contribution to the first Chern class, and this yields more freedom in the choice of the bosonic dimensions to have an overall vanishing first Chern class. In particular, an extension

$$
\hat{S}_{\mathrm{hCS}}=\int_{\mathcal{Y}} \Omega \wedge \operatorname{tr}\left(\hat{\mathcal{A}}^{0,1} \wedge \bar{\partial} \hat{\mathcal{A}}^{0,1}+\frac{2}{3} \hat{\mathcal{A}}^{0,1} \wedge \hat{\mathcal{A}}^{0,1} \wedge \hat{\mathcal{A}}^{0,1}\right)
$$

of the action (1.1) to the supertwistor space $\mathbb{C} P^{3 \mid 4}$ was considered. Here, $\mathcal{Y}$ is a subspace of $\mathbb{C} P^{3 \mid 4}$ parametrized by three complex bosonic coordinates together with their complex conjugate and four (holomorphic) fermionic coordinates, $\Omega$ is a holomorphic measure for bosonic and fermionic coordinates, and $\hat{\mathcal{A}}^{0,1}$ is the $(0,1)$-component of a connection one-form $\hat{\mathcal{A}}$ on a rank $n$ complex vector bundle $\mathcal{E}$ over $\mathbb{C} P^{3 \mid 4}$ depending on both the bosonic and fermionic coordinates. It was shown [2] that there is a bijection between the moduli spaces of hCS theory (1.2) on the supermanifold $\mathbb{C} P^{3 \mid 4} \backslash \mathbb{C} P^{1 \mid 4}$ and of self-dual $\mathcal{N}=4$ super-Yang-Mills (SYM) theory on the space $\mathbb{R}^{4}$ with a metric of signature $(++++)$ or $(--++)$, depending on the reality conditions imposed on the supertwistor space (for related works see [3-10]). It was also demonstrated that the above twistor description allows one to recover Yang-Mills scattering amplitudes, in particular, maximally helicity violating (MHV) ones, ${ }^{2}$ and to clarify the holomorphicity

\footnotetext{
${ }^{1}$ For a definition, see Appendix B.

${ }^{2}$ See [11] for an earlier discussion of this point.
} 
properties $^{3}$ of these amplitudes ${ }^{4}$ and identities appearing in this context $[12-17]$.

Note that Witten described the correspondence between hCS theory on $\mathbb{C} P^{3 \mid 4}$ and anti-self-dual $\mathcal{N}=4$ SYM theory by analysing the sheaf cohomology interpretation of the linearized field equations on the supertwistor space. The main purpose of this paper is to give a more detailed and explicit description of this correspondence for $0 \leq \mathcal{N} \leq 4$ beyond the linearized level. ${ }^{5}$ We will also discuss the supertwistor description of the full $\mathcal{N}=4 \mathrm{SYM}$ theory along the lines proposed in [2]. In fact, we shall consider several special cases of the following general situation. Suppose we are given complex (super)manifolds $X, Y, Z$ and a double fibration

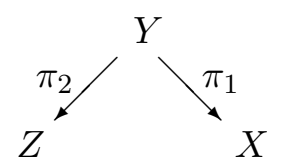

with surjective holomorphic projections $\pi_{1}$ and $\pi_{2}$, then we have a correspondence between $Z$ and $X$, i.e., between points in one space and subspaces of the other one

$$
\begin{aligned}
\text { points } z \text { in } Z & \longleftrightarrow \text { subspaces } \pi_{1}\left(\pi_{2}^{-1}(z)\right) \text { in } X, \\
\text { subspaces } \pi_{2}\left(\pi_{1}^{-1}(x)\right) \text { in } Z & \longleftrightarrow \text { points } x \text { in } X .
\end{aligned}
$$

Using the correspondence (1.4), one can transfer data given on $Z$ to data on $X$ (and vice versa). One may take some analytic objects $h$ on $Z$ (Dolbeault cohomology classes, holomorphic vector bundles, etc.) and transform them to objects $f$ on $X$, which will be constrained by some differential equations, since the pull-back of $h$ to $Y$ has to be constant along the fibres of $\pi_{2}$. The map $\mathcal{P} \mathcal{W}: h \mapsto f$ is called the Penrose-Ward transform. Of course, one can also consider the inverse map $\mathcal{P} \mathcal{W}^{-1}: f \mapsto h$. If there is an antilinear involution $\tau$ (real structure) on $X$, then the set of fixed points of $\tau$ forms a real subspace $X_{\tau}$ of $X$. In the real setup, the double fibration often simplifies to the nonholomorphic fibration

$$
\pi: Z \longrightarrow X_{\tau} \text {. }
$$

This happens when $\pi_{1}^{-1}\left(X_{\tau}\right) \cong Z$ and therefore $\pi_{2}$ becomes a bijection. The correspondence (1.4) is preserved in this case.

\footnotetext{
${ }^{3}$ The unexpected holomorphicity properties of the so-called MHV amplitudes provided the original motivation to study the space $\mathcal{P}^{3 \mid 4}:=\mathbb{C} P^{3 \mid 4} \backslash \mathbb{C} P^{1 \mid 4}$. Holomorphicity is an earmark of the topological B-model, and there is a six-dimensional description of self-dual SYM theory via the twistor correspondence. Thus, one had to find a space which is CY and a complex (super)twistor space of $\mathbb{R}^{4}$ at the same time: $\mathcal{P}^{3 \mid 4}$.

${ }^{4}$ For a preliminary consideration of gravity amplitudes in this context, see [18].

${ }^{5}$ Still, it should be stressed that the supertwistor space is CY only for $\mathcal{N}=4$.
} 
In particular, if field theories are given on the spaces $Z$ and $X$, then a correspondence of the type (1.4) between both spaces can be lifted to a correspondence between solutions to the field equations on $Z$ and solutions to those on $X$. In general, this correspondence will not be one-to-one, since fields are usually defined only up to some (gauge) equivalence. However, one can often establish a one-to-one correspondence between elements of the moduli spaces of theories on $Z$ and those on $X$. This will be specialized later in concrete examples. Our considerations in this paper are based on the results of many authors (see e.g., [19-43] and references therein). More details on twistor theory and the Penrose-Ward transform can be found in the books [44-47].

\section{$2 \quad$ Twistor geometry}

\section{$2.1 \quad$ Local coordinates}

We start from the complex projective space $\mathbb{C} P^{3}$ (the twistor space) with homogeneous coordinates $\left(\omega^{\alpha}, \lambda_{\dot{\alpha}}\right)$ subject to the equivalence relation $\left(\omega^{\alpha}, \lambda_{\dot{\alpha}}\right) \sim\left(t \omega^{\alpha}, t \lambda_{\dot{\alpha}}\right)$ for all nonzero complex $t, \alpha=1,2$ and $\dot{\alpha}=\dot{1}, \dot{2}$. Consider now the space $\mathcal{P}^{3}=\mathbb{C} P^{3} \backslash \mathbb{C} P^{1}$ in which $\left(\lambda_{\dot{\alpha}}\right) \neq 0$. This space can be covered by two patches $\mathcal{U}_{+}\left(\lambda_{\dot{i}} \neq 0\right)$ and $\mathcal{U}_{-}\left(\lambda_{\dot{2}} \neq 0\right)$ with coordinates

$z_{+}^{\alpha}=\frac{\omega^{\alpha}}{\lambda_{\dot{1}}}, \quad z_{+}^{3}=\frac{\lambda_{\dot{2}}}{\lambda_{\dot{1}}}=: \lambda_{+}$on $\mathcal{U}_{+}$and $z_{-}^{\alpha}=\frac{\omega^{\alpha}}{\lambda_{\dot{2}}}, \quad z_{-}^{3}=\frac{\lambda_{\dot{1}}}{\lambda_{\dot{2}}}=: \lambda_{-}$on $\mathcal{U}_{-}$,

related by

$$
z_{+}^{\alpha}=z_{+}^{3} z_{-}^{\alpha} \quad \text { and } \quad z_{+}^{3}=\frac{1}{z_{-}^{3}}
$$

on the overlap $\mathcal{U}_{+} \cap \mathcal{U}_{-}$.

From (2.1) and (2.2), it is obvious that $\mathcal{P}^{3}=\mathcal{U}_{+} \cup \mathcal{U}_{-}$coincides with the total space of the rank 2 holomorphic vector bundle ${ }^{6} \quad \mathcal{P}^{3}=\mathcal{O}(1) \oplus \mathcal{O}(1)$ over $\mathbb{C} P^{1}$,

$$
\mathcal{P}^{3} \longrightarrow \mathbb{C} P^{1},
$$

where the Riemann sphere $\mathbb{C} P^{1}$ is covered by two patches

$$
U_{+}=\mathcal{U}_{+} \cap \mathbb{C} P^{1} \quad \text { and } \quad U_{-}=\mathcal{U}_{-} \cap \mathbb{C} P^{1}
$$

with coordinates $\lambda_{+}$on $U_{+}$and $\lambda_{-}$on $U_{-}, \mathbb{C} P^{1}=U_{+} \cup U_{-}$.

\footnotetext{
${ }^{6}$ The holomorphic line bundle $\mathcal{O}(n)$ over $\mathbb{C} P^{1}$ has the transition function $\lambda_{+}^{n}$ and the first Chern number $n$. See Appendix B for more details.
} 
Special cases of the correspondence (1.3) and (1.4) can be established between the twistor space $Z=\mathbb{C} P^{3}$ and the Graßmann manifold $X=G_{2,4}$ $(\mathbb{C})[44]$ as well as the space $Z=\mathcal{P}^{3}=\mathbb{C} P^{3} \backslash \mathbb{C} P^{1}$ (which we also call twistor space) and $X=\mathbb{C}^{4} \subset G_{2,4}(\mathbb{C})$. In the following, we will focus on the geometry of the latter correspondence.

\subsection{Moduli space of curves}

Holomorphic sections of the complex vector bundle (2.3) are rational curves $\mathbb{C} P_{x}^{1} \hookrightarrow \mathcal{P}^{3}$ defined by the equations

$$
z_{+}^{\alpha}=x^{\alpha \dot{1}}+\lambda_{+} x^{\alpha \dot{2}} \text { for } \lambda_{+} \in U_{+} \quad \text { and } \quad z_{-}^{\alpha}=\lambda_{-} x^{\alpha \dot{1}}+x^{\alpha \dot{2}} \text { for } \lambda_{-} \in U_{-}
$$

and parametrized by moduli $x=\left(x^{\alpha \dot{\alpha}}\right) \in \mathbb{C}^{4}$. Introducing

$$
\left(\lambda_{\dot{\alpha}}^{+}\right):=\left(\begin{array}{c}
1 \\
\lambda_{+}
\end{array}\right) \quad \text { and } \quad\left(\lambda_{\dot{\alpha}}^{-}\right):=\left(\begin{array}{c}
\lambda_{-} \\
1
\end{array}\right)
$$

we can rewrite $(2.5)$ as

$$
z_{ \pm}^{\alpha}=x^{\alpha \dot{\alpha}} \lambda_{\dot{\alpha}}^{ \pm}
$$

These equations allow us to introduce a double fibration ${ }^{7}$

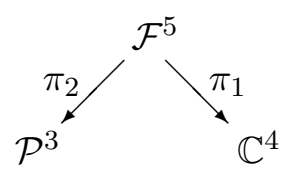

where $\mathcal{F}^{5}:=\mathbb{C}^{4} \times \mathbb{C} P^{1}$. From this diagram, one observes that a point $x=$ $\left(x^{\alpha \dot{\alpha}}\right) \in \mathbb{C}^{4}$ corresponds to the projective line $\mathbb{C} P_{x}^{1}=\pi_{2}\left(\pi_{1}^{-1}(x)\right)$ in $\mathcal{P}^{3}$ given by solutions of $(2.7)$ for fixed $x$, and a point $p=\left(z_{ \pm}^{\alpha}, \lambda_{\dot{\alpha}}^{ \pm}\right) \in \mathcal{P}^{3}$ corresponds $^{8}$ to a totally null anti-self-dual 2-plane $\left(\beta\right.$-plane) $\pi_{1}\left(\pi_{2}^{-1}(p)\right)$ in $\mathbb{C}^{4}$ defined by solutions of (2.7) for fixed $\left(z_{ \pm}^{\alpha}, \lambda_{\dot{\alpha}}^{ \pm}\right)$. The double fibration (2.8) and its $\mathbb{Z}_{2^{-}}$ graded $^{9}$ generalizations play the central rôle in the twistor correspondence.

\section{$2.3 \quad$ Real structures}

Recall that a real structure on a complex manifold $M$ is defined as an antiholomorphic involution $\tau: M \rightarrow M$. To introduce real structures on $\mathcal{P}^{3}$, let

\footnotetext{
${ }^{7}$ Note that we use the same notation $\pi_{1}$ and $\pi_{2}$ for projections in completely different diagrams throughout the paper.

${ }^{8}$ In the literature, $\mathcal{P}^{3}$ is often called the dual twistor space, while the space of totally null self-dual two-planes ( $\alpha$-planes) is called the twistor space.

${ }^{9}$ As usual in theoretical physics, we will use the prefix "super" instead of $\mathbb{Z}_{2}$-graded and do not imply supersymmetry by that term.
} 
us consider three antilinear transformations of dotted and undotted (commuting) spinors,

$$
\begin{aligned}
&\left(\omega^{\alpha}\right) \longmapsto \tau_{\varepsilon}\left(\omega^{\alpha}\right)=\left(\begin{array}{ll}
0 & \varepsilon \\
1 & 0
\end{array}\right)\left(\begin{array}{c}
\bar{\omega}^{1} \\
\bar{\omega}^{2}
\end{array}\right)=\left(\begin{array}{c}
\varepsilon \bar{\omega}^{2} \\
\bar{\omega}^{1}
\end{array}\right)=:\left(\hat{\omega}^{\alpha}\right), \\
&\left(\lambda_{\dot{\alpha}}\right) \longmapsto \tau_{\varepsilon}\left(\lambda_{\dot{\alpha}}\right)=\left(\begin{array}{ll}
0 & \varepsilon \\
1 & 0
\end{array}\right)\left(\begin{array}{c}
\bar{\lambda}_{\dot{i}} \\
\bar{\lambda}_{\dot{2}}
\end{array}\right)=\left(\begin{array}{c}
\varepsilon \bar{\lambda}_{\dot{2}} \\
\bar{\lambda}_{\dot{1}}
\end{array}\right)=:\left(\hat{\lambda}_{\dot{\alpha}}\right), \\
& \tau_{0}\left(\omega^{\alpha}\right)=\left(\bar{\omega}^{\alpha}\right), \quad \tau_{0}\left(\lambda_{\dot{\alpha}}\right)=\left(\bar{\lambda}_{\dot{\alpha}}\right),
\end{aligned}
$$

where $\varepsilon= \pm 1$. For later use, we define furthermore $\left(\hat{\lambda}^{\dot{\alpha}}\right):=\tau\left(\lambda^{\dot{\alpha}}\right)$, i.e., indices are raised as $\lambda^{\dot{\alpha}}=\varepsilon^{\dot{\alpha} \dot{\beta}} \lambda_{\dot{\beta}}$ with $\varepsilon^{\dot{1} \dot{2}}=-\varepsilon^{\dot{2} \dot{1}}=-1$ before the action of $\tau$. The transformations (2.9)-(2.11) define three real structures on $\mathcal{P}^{3}$ which in the coordinates (2.1) are given by the formulæ

$$
\begin{gathered}
\tau_{\varepsilon}\left(z_{+}^{1}, z_{+}^{2}, \lambda_{+}\right)=\left(\frac{\bar{z}_{+}^{2}}{\bar{\lambda}_{+}}, \frac{\varepsilon \bar{z}_{+}^{1}}{\bar{\lambda}_{+}}, \frac{\varepsilon}{\bar{\lambda}_{+}}\right), \quad \tau_{\varepsilon}\left(z_{-}^{1}, z_{-}^{2}, \lambda_{-}\right)=\left(\frac{\varepsilon \bar{z}_{-}^{2}}{\bar{\lambda}_{-}}, \frac{\bar{z}_{-}^{1}}{\bar{\lambda}_{-}}, \frac{\varepsilon}{\bar{\lambda}_{-}}\right), \\
\tau_{0}\left(z_{ \pm}^{1}, z_{ \pm}^{2}, \lambda_{ \pm}\right)=\left(\bar{z}_{ \pm}^{1}, \bar{z}_{ \pm}^{2}, \bar{\lambda}_{ \pm}\right) .
\end{gathered}
$$

It is obvious that the involution $\tau_{-1}$ has no fixed points but does leave invariant projective lines joining $p$ and $\tau_{-1}(p)$ for any $p \in \mathcal{P}^{3}$. On the other hand, the involutions $\tau_{1}$ and $\tau_{0}$ have fixed points which form a three-dimensional real manifold

$$
\mathcal{T}^{3}=\mathbb{R} P^{3} \backslash \mathbb{R} P^{1}
$$

fibred over $S^{1} \cong \mathbb{R} P^{1} \subset \mathbb{C} P^{1}$. The space $\mathcal{T}^{3} \subset \mathcal{P}^{3}$ is called real twistor space. For the real structure $\tau_{1}$, this space is described by the coordinates $\left(z_{ \pm}^{1}, \mathrm{e}^{\mathrm{i} \chi} \bar{z}_{ \pm}^{1}, \mathrm{e}^{\mathrm{i} \chi}\right)$ with $0 \leq \chi<2 \pi$, and for the real structure $\tau_{0}$, the coordinates $\left(z_{ \pm}^{1}, z_{ \pm}^{2}, \lambda_{ \pm}\right)$are real. These two descriptions are equivalent.

We shall concentrate on the real structures $\tau_{ \pm 1}$ since all formulæ for these two cases can be written in a unified form using $\varepsilon= \pm 1$. For instance, an extension of the involution $\tau_{\varepsilon}$ to any function $f\left(x, \lambda_{+}\right)$is defined as

$$
\tau_{\varepsilon}\left(f\left(x, \lambda_{+}\right)\right):=\overline{f\left(\tau_{\varepsilon}\left(x, \lambda_{+}\right)\right)}:=\overline{f\left(\tau_{\varepsilon}(x), \frac{\varepsilon}{\bar{\lambda}_{+}}\right)},
$$

where the bar denotes ordinary complex conjugation. Using this involution, one can impose the condition of invariance under (2.15) on sections (2.7) of the bundle (2.3). Due to (2.5) and (2.12), moduli $\left(x^{\alpha \dot{\alpha}}\right)$ of such sections 
satisfy the equations

$$
x^{2 \dot{2}}=\bar{x}^{1 \dot{1}}=:-\left(\varepsilon x^{4}+\mathrm{i} x^{3}\right) \text { and } x^{2 \dot{1}}=\varepsilon \bar{x}^{1 \dot{2}}=:-\varepsilon\left(x^{2}-\mathrm{i} x^{1}\right),
$$

where $\left(x^{\mu}\right)$ are the real coordinates with $\mu=1, \ldots, 4$. On the other hand, holomorphic sections of the bundle (2.3) which are invariant under the involution $\tau_{0}$ are parametrized by real coordinates

$$
\begin{gathered}
x^{\alpha \dot{\alpha}}=\bar{x}^{\alpha \dot{\alpha}} \\
\Longrightarrow x^{1 \dot{1}}=x^{4}+x^{1}, \quad x^{1 \dot{2}}=x^{2}-x^{3}, \quad x^{2 \dot{1}}=x^{2}+x^{3}, \quad x^{2 \dot{2}}=x^{4}-x^{1} .
\end{gathered}
$$

\subsection{Metric on the moduli space of real curves}

On the space $\mathbb{R}^{4}$ of real holomorphic curves $\mathbb{C} P_{x}^{1} \hookrightarrow \mathcal{P}^{3}$, one can introduce the metric

$$
d s^{2}=\operatorname{det}\left(d x^{\alpha \dot{\alpha}}\right)=g_{\mu \nu} d x^{\mu} d x^{\nu}
$$

with $g=\operatorname{diag}(+1,+1,+1,+1)$ for the involution $\tau_{-1}$ on $\mathcal{P}^{3}$ and $g=$ $\operatorname{diag}(-1,-1,+1,+1)$ for $\tau_{1}$ (and $\left.\tau_{0}\right)$ and $g=\left(g_{\mu \nu}\right)$. Thus, the moduli space of real rational curves of degree one in $\mathcal{P}^{3}$ is the Euclidean space ${ }^{10} \mathbb{R}^{4,0}$ or the Kleinian space $\mathbb{R}^{2,2}$.

Note that, in the Euclidean case, the twistor space $\mathcal{P}^{3}$ is the space

$$
\mathbb{R}^{4} \times \mathbb{C} P^{1} \cong \mathcal{P}^{3}
$$

with the coordinates $\left(x^{\mu}, \lambda_{ \pm}\right)$and one can define a trivial nonholomorphic fibration

$$
\pi: \mathcal{P}^{3} \longrightarrow \mathbb{R}^{4}
$$

over the space $\mathbb{R}^{4}$ with real coordinates $x=\left(x^{\mu}\right)$. Therefore, on the patches $\mathcal{U}_{+}$and $\mathcal{U}_{-}$covering the space $\mathcal{P}^{3}$, one can use the coordinates $\left(x, \lambda_{+}\right)$and $\left(x, \lambda_{-}\right)$, respectively. In the Euclidean case, the double fibration (2.8) simplifies to the fibration (2.20) since $\pi_{1}^{-1}\left(\mathbb{R}^{4}\right) \cong \mathcal{P}^{3} \subset \mathcal{F}^{5}$ and therefore the restriction of the projection $\pi_{2}$ to $\pi_{1}^{-1}\left(\mathbb{R}^{4}\right)$ is a bijection.

The twistor correspondence for the Kleinian case is more complicated. In particular, we have

$$
\mathbb{R}^{4} \times\left(\mathbb{C} P^{1} \backslash S^{1}\right) \cong \mathcal{P}^{3} \backslash \mathcal{P}_{0}
$$

instead of the diffeomorphism (2.19), and one should consider the space $\tilde{\mathcal{P}}^{3}:=\mathcal{P}^{3} \backslash \mathcal{P}_{0}$ with $\mathcal{P}_{0}=\left.\mathcal{P}^{3}\right|_{\left|\lambda_{ \pm}\right|=1} \cong \mathbb{R}^{4} \times S^{1}$ instead of $\mathcal{P}^{3}$ in $(2.20)$. For

\footnotetext{
${ }^{10}$ In our notation, $\mathbb{R}^{p, q}=\left(\mathbb{R}^{p+q}, g\right)$ is the space $\mathbb{R}^{p+q}$ with the metric $g=$ $\operatorname{diag}(\underbrace{-1, \ldots,-1}_{q}, \underbrace{+1, \ldots,+1}_{p})$.
} 
more details on the Kleinian case $\varepsilon=+1$, see Appendix C. To smoothen the discussion in the following, we ignore this subtlety and use always (2.20) implying the restriction to the space $\tilde{\mathcal{P}}^{3}$ in all necessary cases. Furthermore, we will call matrix-valued functions $\tau_{\varepsilon}$-regular, if they are regular ${ }^{11}$ for all values of $\lambda \in D$ in the case $\varepsilon=-1$ and regular for $\lambda \in D$ with $|\lambda| \neq 1$ in the case $\varepsilon=+1$ (and also for the real structure $\tau_{0}$ ), where $D \subseteq \mathbb{C} P^{1}$ is the domain under consideration.

\subsection{Vector fields}

On the complex manifold $\mathcal{P}^{3}$, we have the natural basis $\left(\partial / \partial \bar{z}_{ \pm}^{\alpha}, \partial / \partial \bar{z}_{ \pm}^{3}\right)$ in the space of antiholomorphic vector fields with

$$
\frac{\partial}{\partial \bar{z}_{+}^{\alpha}}=\frac{1}{\bar{z}_{+}^{3}} \frac{\partial}{\partial \bar{z}_{-}^{\alpha}} \quad \text { and } \quad \frac{\partial}{\partial \bar{z}_{+}^{3}}=-\frac{1}{\left(\bar{z}_{+}^{3}\right)^{2}} \frac{\partial}{\partial \bar{z}_{-}^{3}}-\frac{1}{\bar{z}_{+}^{3}} \bar{z}_{-}^{\alpha} \frac{\partial}{\partial \bar{z}_{-}^{\alpha}}
$$

on the intersection $\mathcal{U}_{+} \cap \mathcal{U}_{-}$. In the coordinates $\left(x, \lambda_{+}\right)$on $\mathcal{U}_{+}$, we have ${ }^{12}$

$$
\begin{aligned}
& \frac{\partial}{\partial \bar{z}_{+}^{1}}=\gamma_{+}\left(\frac{\partial}{\partial x^{2 \dot{2}}}-\lambda_{+} \frac{\partial}{\partial x^{2 i}}\right)=\gamma_{+} \lambda_{+}^{\dot{\alpha}} \frac{\partial}{\partial x^{2 \dot{\alpha}}}=: \gamma_{+} \bar{V}_{2}^{+}, \\
& \frac{\partial}{\partial \bar{z}_{+}^{2}}=\varepsilon \gamma_{+}\left(\frac{\partial}{\partial x^{1 \dot{2}}}-\lambda_{+} \frac{\partial}{\partial x^{1 \dot{1}}}\right)=\varepsilon \gamma_{+} \lambda_{+}^{\dot{\alpha}} \frac{\partial}{\partial x^{1 \dot{\alpha}}}=: \varepsilon \gamma_{+} \bar{V}_{1}^{+}, \\
& \frac{\partial}{\partial \bar{z}_{+}^{3}}=\frac{\partial}{\partial \bar{\lambda}_{+}}-\varepsilon \gamma_{+} x^{\alpha \dot{1}} \bar{V}_{\alpha}^{+}=: \bar{V}_{3}^{+}-\varepsilon \gamma_{+} x^{\alpha \dot{1}} \bar{V}_{\alpha}^{+},
\end{aligned}
$$

where

$$
\gamma_{+}=\frac{1}{1-\varepsilon \lambda_{+} \bar{\lambda}_{+}}=\frac{1}{\lambda_{+}^{\dot{\alpha}} \hat{\lambda}_{\dot{\alpha}}^{+}} \quad \text { and } \quad\left(\lambda_{+}^{\dot{\alpha}}\right)=\left(\varepsilon^{\dot{\alpha} \dot{\beta}} \lambda_{\dot{\beta}}^{+}\right)=\left(\begin{array}{c}
-\lambda_{+} \\
1
\end{array}\right)
$$

with the convention ${ }^{13} \varepsilon^{12}:=-\varepsilon^{21}=-1$. Thus, the vector fields

$$
\bar{V}_{\alpha}^{+}=\lambda_{+}^{\dot{\alpha}} \partial_{\alpha \dot{\alpha}} \quad \text { and } \quad \bar{V}_{3}^{+}=\frac{\partial}{\partial \bar{\lambda}_{+}}
$$

form a basis of vector fields of type $(0,1)$ over $\mathcal{U}_{+} \subset \mathcal{P}^{3}$ in coordinates $\left(x, \lambda_{+}\right)$, where $\partial_{\alpha \dot{\alpha}}:=\partial / \partial x^{\alpha \dot{\alpha}}$. The explicit form of the basis of vector fields of type

\footnotetext{
${ }^{11}$ By "regular", we mean smooth and having nonvanishing determinant.

${ }^{12}$ In the case $\varepsilon=+1$, this transformation is only valid for $\left|\lambda_{ \pm}\right| \neq 1$. For more details, see Appendix C.

${ }^{13}$ See Appendix B.
} 
$(0,1)$ on the open set $\mathcal{U}_{-} \subset \mathcal{P}^{3}$ follows from the transformation rules (2.22),

$$
\bar{V}_{\alpha}^{-}=\lambda_{-}^{\dot{\alpha}} \partial_{\alpha \dot{\alpha}} \quad \text { and } \quad \bar{V}_{3}^{-}=\frac{\partial}{\partial \bar{\lambda}_{-}}
$$

where one introduces additionally

$$
\gamma_{-}=-\varepsilon \frac{1}{1-\varepsilon \lambda_{-} \bar{\lambda}_{-}}=\frac{1}{\lambda_{-}^{\dot{\alpha}} \hat{\lambda}_{\dot{\alpha}}^{-}} \quad \text { and } \quad\left(\lambda_{-}^{\dot{\alpha}}\right)=\left(\begin{array}{c}
-1 \\
\lambda_{-}
\end{array}\right) \text {. }
$$

Note that the bases (2.25) and (2.26) of vector fields on $\mathcal{P}^{3}$ are holonomic, i.e., they commute pairwise. Furthermore, in the case $\varepsilon=+1$, the identification (2.23) only holds on $\tilde{\mathcal{P}}^{3}$.

\section{Twistor description of self-dual Yang-Mills fields}

\subsection{Holomorphic bundles over the twistor space}

Consider a rank $n$ holomorphic vector bundle $E$ over the twistor space $\mathcal{P}^{3}$. This bundle is defined by a holomorphic transition function $f_{+-}$on the intersection $\mathcal{U}_{+} \cap \mathcal{U}_{-}$of the two patches covering $\mathcal{P}^{3}=\mathcal{U}_{+} \cup \mathcal{U}_{-}$, i.e., the function $f_{+-}$takes values in the group of nonsingular $n \times n$ matrices annihilated by the vector fields $(2.25)$ of type $(0,1)$

$$
\bar{V}_{\alpha}^{+} f_{+-}=0=\partial_{\bar{\lambda}_{+}} f_{+-} .
$$

In the twistor approach to self-duality, it is assumed that $E$ is topologically trivial and its restriction to any projective line $\mathbb{C} P_{x}^{1} \hookrightarrow \mathcal{P}^{3}$ is holomorphically trivial. These two conditions imply that there are regular matrix-valued functions, $\psi_{+}$on $\mathcal{U}_{+}$and $\psi_{-}$on $\mathcal{U}_{-}$, such that

$$
f_{+-}=\psi_{+}^{-1} \psi_{-}
$$

and

$$
\partial_{\bar{\lambda}_{+}} \psi_{+}=0=\partial_{\bar{\lambda}_{-}} \psi_{-}
$$

i.e., $\psi_{+}$and $\psi_{-}$are smooth functions of $x \in \mathbb{R}^{4}$ and holomorphic functions of $\lambda_{+}$and $\lambda_{-}$, respectively. 


\subsection{Gauge potentials}

From the holomorphicity condition (3.1) together with (3.2), it follows that

$$
\psi_{+} \bar{V}_{\alpha}^{+} \psi_{+}^{-1}=\psi_{-} \bar{V}_{\alpha}^{+} \psi_{-}^{-1}
$$

on $\mathcal{U}_{+} \cap \mathcal{U}_{-}$. One can expand $\psi_{+}, \psi_{+}^{-1}$ and $\psi_{-}, \psi_{-}^{-1}$ as power series in $\lambda_{+}$ and $\lambda_{-}=\lambda_{+}^{-1}$, respectively. Upon substituting the expansions into equations (3.4), one sees that both sides in (3.4) must be linear in $\lambda_{+}$and one can introduce Lie-algebra valued fields $\left(A_{\alpha \dot{\alpha}}\right)$ depending only on $x$ by the formulæ

$$
\lambda_{+}^{\dot{\alpha}} A_{\alpha \dot{\alpha}}=\lambda_{+}^{\dot{\alpha}} \psi_{+} \partial_{\alpha \dot{\alpha}} \psi_{+}^{-1}=\lambda_{+}^{\dot{\alpha}} \psi_{-} \partial_{\alpha \dot{\alpha}} \psi_{-}^{-1},
$$

where $\left(\lambda_{+}^{\dot{\alpha}}\right)$ is given in (2.24). The matrix-valued functions $\left(A_{\alpha \dot{\alpha}}(x)\right)$ can be identified with the components of a gauge potential $A_{\alpha \dot{\alpha}} d x^{\alpha \dot{\alpha}}$ on $\mathbb{R}^{4}$ with the metric $\left(g_{\mu \nu}\right)=\operatorname{diag}(-\varepsilon,-\varepsilon,+1,+1)$. Note that $A_{\alpha \dot{\alpha}} d x^{\alpha \dot{\alpha}}$ will be an antihermitean $n \times n$ matrix if $\psi_{ \pm}$satisfies the following condition ${ }^{14}$

$$
\psi_{+}^{-1}\left(x, \lambda_{+}\right)=\left(\psi_{-}\left(x, \frac{\varepsilon}{\bar{\lambda}_{-}}\right)\right)^{\dagger} .
$$

The antihermitean gauge potential components can be calculated from (3.5) as

$$
A_{1 \dot{2}}=\left.\psi_{+} \partial_{1 \dot{2}} \psi_{+}^{-1}\right|_{\lambda_{+}=0}=-\varepsilon A_{2 \dot{1}}^{\dagger}, \quad A_{2 \dot{2}}=\left.\psi_{+} \partial_{2 \dot{2}} \psi_{+}^{-1}\right|_{\lambda_{+}=0}=-A_{1 \dot{1}}^{\dagger} .
$$

Combining (3.4) and (3.5), we introduce matrix-valued functions ${ }^{15}$

$$
\left.A_{\alpha}^{+}:=\bar{V}_{\alpha}^{+}\right\lrcorner A^{0,1}=\psi_{+} \bar{V}_{\alpha}^{+} \psi_{+}^{-1}=\lambda_{+}^{\dot{\alpha}} A_{\alpha \dot{\alpha}}
$$

where $\lambda_{+}^{\dot{\alpha}}$ are given in (2.24). Analogously, one can introduce the component $A_{\bar{\lambda}_{+}}$, but it will vanish as ${ }^{16}$

$$
A_{\bar{\lambda}_{+}}=\psi_{+} \partial_{\bar{\lambda}_{+}} \psi_{+}^{-1}=0
$$

\footnotetext{
${ }^{14}$ Here $\dagger$ means Hermitean conjugation.

${ }^{15}$ Here $\left.V\right\lrcorner A^{0,1}$ denotes the interior product of a vector field $V$ and a $(0,1)$-form $A^{0,1}$.

${ }^{16}$ Note also that $A_{\alpha}^{+}$and $A_{\bar{\lambda}_{+}}$are components of $A^{0,1}$.
} 


\subsection{Linear system and SDYM equations}

Let us rewrite (3.8) together with (3.3) in the form

$$
\begin{aligned}
\left(\bar{V}_{\alpha}^{+}+A_{\alpha}^{+}\right) \psi_{+} & =0 \\
\partial_{\bar{\lambda}_{+}} \psi_{+} & =0
\end{aligned}
$$

with similar equations for $\psi_{-}$. The compatibility conditions of this linear system are

$$
\left[\bar{V}_{\alpha}^{+}+A_{\alpha}^{+}, \bar{V}_{\beta}^{+}+A_{\beta}^{+}\right]=\lambda_{+}^{\dot{\alpha}} \lambda_{+}^{\dot{\beta}}\left[\partial_{\alpha \dot{\alpha}}+A_{\alpha \dot{\alpha}}, \partial_{\beta \dot{\beta}}+A_{\beta \dot{\beta}}\right]=: \lambda_{+}^{\dot{\alpha}} \lambda_{+}^{\dot{\beta}} F_{\alpha \dot{\alpha}, \beta \dot{\beta}}=0 .
$$

To be satisfied for all $\left(\lambda_{+}^{\dot{\alpha}}\right)$, this equation has to vanish to all orders in $\lambda_{+}$separately, from which we obtain the self-dual Yang-Mills (SDYM) equations

$$
F_{1 \dot{1}, 2 \dot{1}}=0, \quad F_{1 \dot{2}, 2 \dot{2}}=0, \quad F_{1 \dot{1}, 2 \dot{2}}+F_{1 \dot{2}, 2 \dot{1}}=0
$$

for a gauge potential $\left(A_{\alpha \dot{\alpha}}\right)$. It is convenient to introduce the notation

$$
F_{\alpha \dot{\alpha}, \beta \dot{\beta}}=\varepsilon_{\alpha \beta} f_{\dot{\alpha} \dot{\beta}}+\varepsilon_{\dot{\alpha} \dot{\beta}} f_{\alpha \beta},
$$

in which the SDYM equations are rewritten as

$$
f_{\dot{\alpha} \dot{\beta}}=0
$$

\subsection{Gauge equivalent linear systems}

Note that in (3.2), we have chosen special trivializations ${ }^{17} \quad \psi_{ \pm}$of $E$ over $\mathcal{U}_{ \pm}$such that (3.3) and, therefore (3.9) were satisfied. However, one can consider more general trivializations $\left\{\hat{\psi}_{ \pm}\right\}$of $E$ such that

$$
f_{+-}=\psi_{+}^{-1} \psi_{-}=\hat{\psi}_{+}^{-1} \hat{\psi}_{-}
$$

\footnotetext{
${ }^{17}$ On the bundle $E$, the Čech fibre coordinates $\chi_{ \pm}\left(z_{ \pm}^{\alpha}, \lambda_{ \pm}\right)$are related by $\chi_{+}=f_{+-} \chi_{-}$ on $\mathcal{U}_{+} \cap \mathcal{U}_{-}$. At the same time, the matrices $\psi_{+}$and $\psi_{-}$are matrix fundamental solutions of equations (3.10) and (3.11), i.e., the columns of $\psi_{ \pm}$form smooth frame fields for $E$ over $\mathcal{U}_{ \pm}$. In other words, regular matrix-valued functions $\psi_{ \pm}$define a trivialization of $E$ over $\mathcal{U}_{ \pm}$such that $\psi_{ \pm} \chi_{ \pm}$are sections of $E$ holomorphic w.r.t. a new complex structure (defined by $\bar{\partial}+A^{0,1}$ ). They are related by $\psi_{+} \chi_{+}=\psi_{-} \chi_{-}$on $\mathcal{U}_{+} \cap \mathcal{U}_{-}$.
} 
and $\partial_{\bar{\lambda}_{ \pm}} \hat{\psi}_{ \pm} \neq 0$, i.e., $\hat{\psi}_{ \pm}=\hat{\psi}_{ \pm}\left(x, \lambda_{ \pm}, \bar{\lambda}_{ \pm}\right)$are regular matrix-valued functions smooth in all coordinates on $\mathcal{U}_{ \pm}$. From (3.16) it follows that

$$
\varphi:=\psi_{+} \hat{\psi}_{+}^{-1}=\psi_{-} \hat{\psi}_{-}^{-1}
$$

is a globally defined regular matrix-valued function on $\mathcal{P}^{3}$ and therefore the above two trivializations are related by the gauge transformation

$$
\psi_{ \pm} \longmapsto \hat{\psi}_{ \pm}=\varphi^{-1} \psi_{ \pm}
$$

In general trivializations $\left\{\hat{\psi}_{ \pm}\right\}$, we have

$$
\begin{aligned}
\hat{A}_{\alpha}^{+} & :=\hat{\psi}_{+} \bar{V}_{\alpha}^{+} \hat{\psi}_{+}^{-1}=\hat{\psi}_{-} \bar{V}_{\alpha}^{+} \hat{\psi}_{-}^{-1}=\varphi^{-1}\left(\psi_{ \pm} \bar{V}_{\alpha}^{+} \psi_{ \pm}^{-1}\right) \varphi+\varphi^{-1} \bar{V}_{\alpha}^{+} \varphi \\
& =\varphi^{-1} A_{\alpha}^{+} \varphi+\varphi^{-1} \bar{V}_{\alpha}^{+} \varphi \\
\hat{A}_{\bar{\lambda}_{+}} & :=\hat{\psi}_{+} \partial_{\bar{\lambda}_{+}} \hat{\psi}_{+}^{-1}=\hat{\psi}_{-} \partial_{\bar{\lambda}_{+}} \hat{\psi}_{-}^{-1}=\varphi^{-1} \partial_{\bar{\lambda}_{+}} \varphi
\end{aligned}
$$

where the last equality in (3.20) follows from (3.11) and (3.9). Equations (3.19) and (3.20) can be rewritten in the form of a linear system

$$
\begin{array}{r}
\left(\bar{V}_{\alpha}^{+}+\hat{A}_{\alpha}^{+}\right) \hat{\psi}_{+}=0, \\
\left(\partial_{\bar{\lambda}_{+}}+\hat{A}_{\bar{\lambda}_{+}}\right) \hat{\psi}_{+}=0,
\end{array}
$$

which is gauge equivalent to the linear system (3.10) and (3.11).

The compatibility conditions of this linear system are in fact the field equations of hCS theory on the space $\mathcal{P}^{3}$, and, e.g., on $\mathcal{U}_{+}$, they take the form

$$
\begin{aligned}
\bar{V}_{\alpha}^{+} \hat{A}_{\beta}^{+}-\bar{V}_{\beta}^{+} \hat{A}_{\alpha}^{+}+\left[\hat{A}_{\alpha}^{+}, \hat{A}_{\beta}^{+}\right]=0, \\
\partial_{\bar{\lambda}_{+}} \hat{A}_{\alpha}^{+}-\bar{V}_{\alpha}^{+} \hat{A}_{\bar{\lambda}_{+}}+\left[\hat{A}_{\bar{\lambda}_{+}}, \hat{A}_{\alpha}^{+}\right]=0 .
\end{aligned}
$$

Thus, we have a relation between hCS on $\mathcal{P}^{3}$ and SDYM on the moduli space $\mathbb{R}^{4}$ of real holomorphic sections of the fibration $\mathcal{P}^{3} \rightarrow \mathbb{C} P^{1}$.

More explicitly, from the formulæ (3.8) and (3.9), we obtain

$$
A_{\alpha \mathrm{i}}=-\oint_{S^{1}} \frac{d \lambda_{+}}{2 \pi \mathrm{i}} \frac{A_{\alpha}^{+}}{\lambda_{+}^{2}} \quad \text { and } \quad A_{\alpha \dot{2}}=\oint_{S^{1}} \frac{d \lambda_{+}}{2 \pi \mathrm{i}} \frac{A_{\alpha}^{+}}{\lambda_{+}}
$$

where the contour $S^{1}=\left\{\lambda_{+} \in \mathbb{C} P^{1}:\left|\lambda_{+}\right|=r<1\right\}$ encircles $\lambda_{+}=0$. Using (3.8), one can easily show the equivalence of (3.25) and (3.7). The formulæ 
(3.25) define the Penrose-Ward transform

$$
\mathcal{P W}:\left(A_{\alpha}^{+}, A_{\bar{\lambda}_{+}}=0\right) \longmapsto\left(A_{\alpha \dot{\alpha}}\right)
$$

which together with a preceding gauge transformation

$$
\left(\hat{A}_{\alpha}^{+}, \hat{A}_{\bar{\lambda}_{+}}\right) \stackrel{\varphi}{\longmapsto}\left(A_{\alpha}^{+}, A_{\bar{\lambda}_{+}}=0\right)
$$

maps solutions $\left(\hat{A}_{\alpha}^{+}, \hat{A}_{\bar{\lambda}_{+}}\right)$of the field equations of hCS theory on $\mathcal{P}^{3}$ to solutions $\left(A_{\alpha \dot{\alpha}}\right)$ of the SDYM equations on $\mathbb{R}^{4}$. Conversely, by formulæ (3.8) and (3.9), any solution $\left(A_{\alpha \dot{\alpha}}\right)$ of the SDYM equations corresponds to a solution $\left(\hat{A}_{\alpha}^{+}, \hat{A}_{\bar{\lambda}_{+}}\right)$of the field equations of hCS theory on $\mathcal{P}^{3}$ which directly defines the inverse Penrose-Ward transform $\mathcal{P} \mathcal{W}^{-1}$. Note that gauge transformations $^{18}$ of $\left(\hat{A}_{\alpha}^{+}, \hat{A}_{\bar{\lambda}_{+}}\right)$on $\mathcal{P}^{3}$ and $\left(A_{\alpha \dot{\alpha}}\right)$ on $\mathbb{R}^{4}$ do not change the transition function $f_{+-}$of the holomorphic bundle $E \rightarrow \mathcal{P}^{3}$. Therefore, we have a oneto-one correspondence between gauge equivalence classes of solutions (i.e., points of the moduli spaces) to the field equations of hCS theory ${ }^{19}$ on $\mathcal{P}^{3}$ and the SDYM equations on $\mathbb{R}^{4}$.

Recall that the trivializations defined by (3.2) and (3.3) correspond to holomorphic triviality of the bundle $\left.E\right|_{\mathbb{C} P_{x}^{1}}$ for any $\mathbb{C} P_{x}^{1} \hookrightarrow \mathcal{P}^{3}$. Similarly, we may consider restrictions of $E$ to fibres $\mathbb{C}_{\lambda}^{2}$ of the fibration $\mathcal{P}^{3} \rightarrow \mathbb{C} P^{1}$. All these restrictions are holomorphically trivial due to the contractibility of $\mathbb{C}_{\lambda}^{2}$ for any $\lambda \in \mathbb{C} P^{1}$. Therefore, there exist regular matrix-valued functions $\tilde{\psi}_{ \pm}\left(z_{ \pm}^{\alpha}, \lambda_{ \pm}, \bar{\lambda}_{ \pm}\right)$depending holomorphically on $z_{ \pm}^{\alpha}$ (and nonholomorphically on $\left.\lambda_{ \pm}\right)$such that

$$
f_{+-}=\psi_{+}^{-1} \psi_{-}=\tilde{\psi}_{+}^{-1} \tilde{\psi}_{-},
$$

and $\tilde{\varphi}:=\psi_{+} \tilde{\psi}_{+}^{-1}=\psi_{-} \tilde{\psi}_{-}^{-1}$ defines a gauge transformation

$$
\left(A_{\alpha}^{+}, A_{\bar{\lambda}_{+}}=0\right) \stackrel{\tilde{\varphi}}{\longmapsto}\left(\tilde{A}_{\alpha}^{+}=0, \tilde{A}_{\bar{\lambda}_{+}}\right)
$$

to a special trivialization in which only $\tilde{A}_{\bar{\lambda}_{+}} \neq 0$ and $\bar{V}_{\alpha}^{+}\left(\tilde{A}_{\bar{\lambda}_{+}}\right)=0$. Certainly, both trivializations in (3.28) and (3.29) are gauge equivalent to the general one given by (3.16)-(3.22). This kind of equivalence will be particularly useful in the supercase.

\footnotetext{
${ }^{18}$ There are two gauge transformations for gauge potentials on two different spaces present in the discussion.

${ }^{19}$ Note that in this twistor correspondence, it is assumed that for solutions of hCS theory, there exists a gauge in which $A_{\bar{\lambda}_{ \pm}}=0$. This is equivalent to the holomorphic triviality of the bundle $E \rightarrow \mathcal{P}^{3}$ on $\mathbb{C} P_{x}^{1} \hookrightarrow \mathcal{P}^{3}$. Solutions $\left(A_{\alpha}^{ \pm}, A_{\bar{\lambda}_{ \pm}}=0\right)$ form a subset in the set of all solutions of the hCS theory on $\mathcal{P}^{3}$, and we imply the restriction to this subset when speaking of solutions to hCS theory in the context of a twistor correspondence.
} 


\section{Supertwistor geometry}

\subsection{Coordinates}

A superextension of the twistor space $\mathbb{C} P^{3}$ is the supermanifold $\mathbb{C} P^{3 \mid \mathcal{N}}$ with homogeneous coordinates $\left(\omega^{\alpha}, \lambda_{\dot{\alpha}}, \eta_{i}\right)$ subject to the identification $\left(\omega^{\alpha}, \lambda_{\dot{\alpha}}\right.$, $\left.\eta_{i}\right) \sim\left(t \omega^{\alpha}, t \lambda_{\dot{\alpha}}, t \eta_{i}\right)$ for any nonzero complex scalar $t$. Here, $\left(\omega^{\alpha}, \lambda_{\dot{\alpha}}\right)$ are homogeneous coordinates on $\mathbb{C} P^{3}$ and $\eta_{i}$ with $i=1, \ldots, \mathcal{N}$ are Graßmann variables. Interestingly, this supertwistor space is a $\mathrm{CY}$ supermanifold in the case $\mathcal{N}=4$, and one may consider B-type open topological strings living in this space [2].

As in Section 2, we consider the space $\mathcal{P}^{3}=\mathbb{C} P^{3} \backslash \mathbb{C} P^{1}=\mathcal{O}(1) \oplus \mathcal{O}(1)$ and its superextension $\mathcal{P}^{3 \mid \mathcal{N}}$ covered by two patches, $\mathcal{P}^{3 \mid \mathcal{N}}=\mathbb{C} P^{3 \mid \mathcal{N}} \backslash \mathbb{C} P^{1 \mid \mathcal{N}}=$ $\hat{\mathcal{U}}_{+} \cup \hat{\mathcal{U}}_{-}$, with even coordinates (2.1) and odd coordinates

$$
\eta_{i}^{+}=\frac{\eta_{i}}{\lambda_{i}} \text { on } \hat{\mathcal{U}}_{+} \quad \text { and } \quad \eta_{i}^{-}=\frac{\eta_{i}}{\lambda_{\dot{2}}} \text { on } \hat{\mathcal{U}}_{-}
$$

related by

$$
\eta_{i}^{+}=z_{+}^{3} \eta_{i}^{-}
$$

on $\hat{\mathcal{U}}_{+} \cap \hat{\mathcal{U}}_{-}$. We see from (4.1) and (4.2) that the fermionic coordinates are sections of ${ }^{20} \Pi \mathcal{O}(1)$. The supermanifold $\mathcal{P}^{3 \mid \mathcal{N}}$ is fibred over $\mathbb{C} P^{1}$,

$$
\mathcal{P}^{3 \mid \mathcal{N}} \longrightarrow \mathbb{C} P^{1 \mid 0}
$$

with superspaces $\mathbb{C}_{\lambda}^{2 \mid \mathcal{N}}$ as fibres over $\lambda \in \mathbb{C} P^{1 \mid 0}=\mathbb{C} P^{1}$. We also have a fibration

$$
\mathcal{P}^{3 \mid \mathcal{N}} \longrightarrow \mathbb{C} P^{1 \mid \mathcal{N}}
$$

with $\mathbb{C}_{\lambda, \eta}^{2 \mid 0}$ as fibres over $(\lambda, \eta) \in \mathbb{C} P^{1 \mid \mathcal{N}}$.

\subsection{Spaces of supermoduli and chirality}

Holomorphic sections of the bundle (4.3) are rational curves $\mathbb{C} P_{x_{\mathrm{R}}, \eta}^{1} \hookrightarrow \mathcal{P}^{3 \mid \mathcal{N}}$ defined by the equations ${ }^{21}$

$$
\begin{array}{lll}
z_{+}^{\alpha}=x_{\mathrm{R}}^{\alpha \dot{\alpha}} \lambda_{\dot{\alpha}}^{+}, & \eta_{i}^{+}=\eta_{i}^{\dot{\alpha}} \lambda_{\dot{\alpha}}^{+} \text {for } \quad\left(\lambda_{\dot{\alpha}}^{+}\right)^{\top}=\left(1, \lambda_{+}\right), \quad \lambda_{+} \in U_{+}, \\
z_{-}^{\alpha}=x_{\mathrm{R}}^{\alpha \dot{\alpha}} \lambda_{\dot{\alpha}}^{-}, & \eta_{i}^{-}=\eta_{i}^{\dot{\alpha}} \lambda_{\dot{\alpha}}^{-} \text {for } \quad\left(\lambda_{\dot{\alpha}}^{-}\right)^{\top}=\left(\lambda_{-}, 1\right), \quad \lambda_{-} \in U_{-}
\end{array}
$$

\footnotetext{
${ }^{20}$ The operator $\Pi$ inverts the parity of fibre coordinates, see Appendix B.

${ }^{21}$ Here $T$ means the transpose.
} 
and parametrized by supermoduli $\left(x_{\mathrm{R}}, \eta\right)=\left(x_{\mathrm{R}}^{\alpha \dot{\alpha}}, \eta_{i}^{\dot{\alpha}}\right) \in \mathbb{C}^{4 \mid 2 \mathcal{N}}=: \mathcal{M}_{\mathrm{R}}^{4 \mid 2 \mathcal{N}}$. The space $\mathcal{M}_{\mathrm{R}}^{4 \mid 2 \mathcal{N}}$ is called antichiral superspace. Equations (4.5) define a supertwistor correspondence via a double fibration

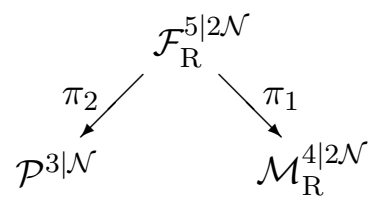

where $\mathcal{F}_{\mathrm{R}}^{5 \mid 2 \mathcal{N}}:=\mathcal{M}_{\mathrm{R}}^{4 \mid 2 \mathcal{N}} \times \mathbb{C} P^{1}$, a point $\left(x_{\mathrm{R}}, \eta\right)=\left(x_{\mathrm{R}}^{\alpha \dot{\alpha}}, \eta_{i}^{\dot{\alpha}}\right) \in \mathcal{M}_{\mathrm{R}}^{4 \mid 2 \mathcal{N}}$ corresponds to the sphere $\mathbb{C} P_{x_{\mathrm{R}}, \eta}^{1}=\pi_{2}\left(\pi_{1}^{-1}\left(x_{\mathrm{R}}, \eta\right)\right) \hookrightarrow \mathcal{P}^{3 \mid \mathcal{N}}$, and a point $p=\left(z_{ \pm}^{\alpha}\right.$, $\left.\lambda_{ \pm}, \eta_{i}^{ \pm}\right) \in \mathcal{P}^{3 \mid \mathcal{N}}$ corresponds to a null $\beta_{\mathrm{R}}$-superplane $\pi_{1}\left(\pi_{2}^{-1}(p)\right) \hookrightarrow \mathcal{M}_{\mathrm{R}}^{4 \mid 2 \mathcal{N}}$ of dimension $(2 \mid \mathcal{N})$.

Holomorphic sections of the bundle (4.4) are spaces $\mathbb{C} P_{x_{\mathrm{L}}, \theta}^{1 \mid \mathcal{N}} \hookrightarrow \mathcal{P}^{3 \mid \mathcal{N}}$ defined by the equations

$$
z_{ \pm}^{\alpha}=x_{\mathrm{L}}^{\alpha \dot{\alpha}} \lambda_{\dot{\alpha}}^{ \pm}-2 \theta^{\alpha i} \eta_{i}^{ \pm} \quad \text { with }\left(\lambda_{\dot{\alpha}}^{ \pm}, \eta_{i}^{ \pm}\right) \in \hat{\mathcal{U}}_{ \pm} \cap \mathbb{C} P^{1 \mid \mathcal{N}}
$$

and parametrized by supermoduli $\left(x_{\mathrm{L}}, \theta\right)=\left(x_{\mathrm{L}}^{\alpha \dot{\alpha}}, \theta^{\alpha i}\right) \in \mathbb{C}^{4 \mid 2 \mathcal{N}}=: \mathcal{M}_{\mathrm{L}}^{4 \mid 2 \mathcal{N}}$. The space $\mathcal{M}_{\mathrm{L}}^{4 \mid 2 \mathcal{N}}$ is called chiral superspace. Equations (4.7) define another supertwistor correspondence,

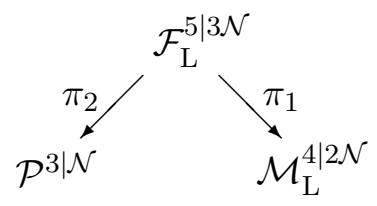

where $\mathcal{F}_{\mathrm{L}}^{5 \mid 3 \mathcal{N}}:=\mathcal{M}_{\mathrm{L}}^{4 \mid 2 \mathcal{N}} \times \mathbb{C} P^{1 \mid \mathcal{N}}$, and a point $\left(x_{\mathrm{L}}, \theta\right)=\left(x_{\mathrm{L}}^{\alpha \dot{\alpha}}, \theta^{\alpha i}\right) \in \mathcal{M}_{\mathrm{L}}^{4 \mid 2 \mathcal{N}}$ corresponds to the supersphere $\mathbb{C} P_{x_{\mathrm{L}}, \theta}^{1 \mid \mathcal{N}}=\pi_{2}\left(\pi_{1}^{-1}\left(x_{\mathrm{L}}, \theta\right)\right) \hookrightarrow \mathcal{P}^{3 \mid \mathcal{N}}$, and a point $p \in \mathcal{P}^{3 \mid \mathcal{N}}$ corresponds to a null $\beta_{\mathrm{L}}$-superplane $\pi_{1}\left(\pi_{2}^{-1}(p)\right) \hookrightarrow \mathcal{M}_{\mathrm{L}}^{4 \mid 2 \mathcal{N}}$ of dimensions $(2 \mid 2 \mathcal{N})$.

From (4.5) and (4.7), it follows that

$$
x_{\mathrm{R}}^{\alpha \dot{\alpha}}=x^{\alpha \dot{\alpha}}-\theta^{\alpha i} \eta_{i}^{\dot{\alpha}}
$$

and

$$
x_{\mathrm{L}}^{\alpha \dot{\alpha}}=x^{\alpha \dot{\alpha}}+\theta^{\alpha i} \eta_{i}^{\dot{\alpha}}
$$

where $\left(x^{\alpha \dot{\alpha}}\right) \in \mathbb{C}^{4 \mid 0}$ are "symmetric" (nonchiral) bosonic coordinates. In the following, we will continue labelling chiral objects by a subscript $L$ for left-handed or chiral and a subscript $R$ for right-handed or antichiral ones. 
Substituting (4.9) into (4.5), we obtain the equations

$$
z_{ \pm}^{\alpha}=x^{\alpha \dot{\alpha}} \lambda_{\dot{\alpha}}^{ \pm}-\theta^{\alpha i} \eta_{i}^{\dot{\alpha}} \lambda_{\dot{\alpha}}^{ \pm} \quad \text { and } \quad \eta_{i}^{ \pm}=\eta_{i}^{\dot{\alpha}} \lambda_{\dot{\alpha}}^{ \pm}
$$

defining degree one curves $\mathbb{C} P_{x, \theta, \eta}^{1} \hookrightarrow \mathcal{P}^{3 \mid \mathcal{N}}$ parametrized by supermoduli $(x, \theta, \eta)=\left(x^{\alpha \dot{\alpha}}, \theta^{\alpha i}, \eta_{i}^{\dot{\alpha}}\right) \in \mathbb{C}^{4 \mid 4 \mathcal{N}}$. Therefore, we obtain a double fibration

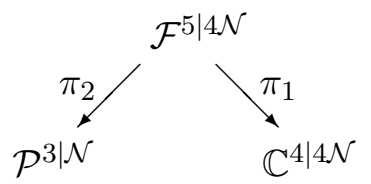

with coordinates

$$
\begin{gathered}
\left(x^{\alpha \dot{\alpha}}, \lambda_{\dot{\alpha}}^{ \pm}, \theta^{\alpha i}, \eta_{i}^{\dot{\alpha}}\right) \quad \text { on } \mathcal{F}^{5 \mid 4 \mathcal{N}}:=\mathbb{C}^{4 \mid 4 \mathcal{N}} \times \mathbb{C P}^{1}, \\
\left(x^{\alpha \dot{\alpha}}, \theta^{\alpha i}, \eta_{i}^{\dot{\alpha}}\right) \quad \text { on } \mathbb{C}^{4 \mid 4 \mathcal{N}},
\end{gathered}
$$

and

$$
z_{ \pm}^{\alpha}=\left(x^{\alpha \dot{\alpha}}-\theta^{\alpha i} \eta_{i}^{\dot{\alpha}}\right) \lambda_{\dot{\alpha}}^{ \pm}, \quad \lambda_{\dot{\alpha}}^{ \pm}, \quad \eta_{i}^{ \pm}=\eta_{i}^{\dot{\alpha}} \lambda_{\dot{\alpha}}^{ \pm} \quad \text { on } \mathcal{P}^{3 \mid \mathcal{N}}
$$

The definition of the projection $\pi_{1}$ is obvious and $\pi_{2}$ is defined by (4.15) and (4.9).

The double fibration (4.12) generalizes both (4.6) and (4.8) and defines the following twistor correspondence: a point $(x, \theta, \eta)=\left(x^{\alpha \dot{\alpha}}, \theta^{\alpha i}, \eta_{i}^{\dot{\alpha}}\right) \in \mathbb{C}^{4 \mid 4 \mathcal{N}}$ corresponds to the projective line $\mathbb{C} P_{x, \theta, \eta}^{1}=\pi_{2}\left(\pi_{1}^{-1}(x, \theta, \eta)\right) \hookrightarrow \mathcal{P}^{3 \mid \mathcal{N}}$, and a point $p \in \mathcal{P}^{3 \mid \mathcal{N}}$ corresponds to a totally null $\beta$-superplane $\pi_{1}\left(\pi_{2}^{-1}(p)\right) \hookrightarrow \mathbb{C}^{4 \mid 4 \mathcal{N}}$ of dimensions $(2 \mid 3 \mathcal{N})$.

\subsection{Vector fields}

Note that one can project from $\mathcal{F}^{5 \mid 4 \mathcal{N}}$ onto $\mathcal{P}^{3 \mid \mathcal{N}}$ in two steps: first from $\mathcal{F}^{5 \mid 4 \mathcal{N}}$ onto $\mathcal{F}_{\mathrm{R}}^{5 \mid 2 \mathcal{N}}$, which is given in coordinates by

$$
\left(x^{\alpha \dot{\alpha}}, \lambda_{\dot{\alpha}}^{ \pm}, \theta^{\alpha i}, \eta_{i}^{\dot{\alpha}}\right) \longrightarrow\left(x_{\mathrm{R}}^{\alpha \dot{\alpha}}, \lambda_{\dot{\alpha}}^{ \pm}, \eta_{i}^{\dot{\alpha}}\right)
$$

with the $x_{\mathrm{R}}^{\alpha \dot{\alpha}}$ from (4.9), and then from $\mathcal{F}_{\mathrm{R}}^{5 \mid 2 \mathcal{N}}$ onto $\mathcal{P}^{3 \mid \mathcal{N}}$, which is given in coordinates by

$$
\left(x_{\mathrm{R}}^{\alpha \dot{\alpha}}, \lambda_{\dot{\alpha}}^{ \pm}, \eta_{i}^{\dot{\alpha}}\right) \longrightarrow\left(x_{\mathrm{R}}^{\alpha \dot{\alpha}} \lambda_{\dot{\alpha}}^{ \pm}, \lambda_{\dot{\alpha}}^{ \pm}, \eta_{i}^{\dot{\alpha}} \lambda_{\dot{\alpha}}^{ \pm}\right) .
$$

The tangent spaces to the $(0 \mid 2 \mathcal{N})$-dimensional leaves of the fibration (4.16) are spanned by the vector fields

$$
D_{\alpha i}=\frac{\partial}{\partial \theta^{\alpha i}}+\eta_{i}^{\dot{\alpha}} \frac{\partial}{\partial x^{\alpha \dot{\alpha}}}=: \partial_{\alpha i}+\eta_{i}^{\dot{\alpha}} \partial_{\alpha \dot{\alpha}}
$$

on $\mathbb{C}^{4 \mid 4 \mathcal{N}} \subset \mathcal{F}^{5 \mid 4 \mathcal{N}}$. The coordinates $x_{\mathrm{R}}^{\alpha \dot{\alpha}}, \lambda_{\dot{\alpha}}^{ \pm}$and $\eta_{i}^{\dot{\alpha}}$ belong to the kernel of these vector fields which are also tangent to the fibres of the projection 
$\mathbb{C}^{4 \mid 4 \mathcal{N}} \rightarrow \mathbb{C}^{4 \mid 2 \mathcal{N}}$ onto the antichiral superspace. The tangent spaces to the $(2 \mid \mathcal{N})$-dimensional leaves of the projection $(4.17)$ are spanned by the vector fields ${ }^{22}$

$$
\begin{aligned}
& \bar{V}_{\alpha}^{ \pm}=\lambda_{ \pm}^{\dot{\alpha}} \partial_{\alpha \dot{\alpha}}^{R} \\
& \bar{\partial}_{ \pm}^{i}=\lambda_{ \pm}^{\dot{\alpha}} \partial_{\dot{\alpha}}^{i} \quad \text { with } \partial_{\dot{\alpha}}^{i}=\frac{\partial}{\partial \eta_{i}^{\dot{\alpha}}}
\end{aligned}
$$

where $\partial_{\alpha \dot{\alpha}}^{R}=\partial / \partial x_{\mathrm{R}}^{\alpha \dot{\alpha}}$.

\subsection{Twistor correspondence for a real superspace}

Let us now discuss the action of the antilinear involutions (2.9)-(2.11) on fermionic coordinates. In the Kleinian case, we can simply define

$$
\tau_{1}\left(\begin{array}{c}
\theta^{1 i} \\
\theta^{2 i}
\end{array}\right)=\left(\begin{array}{c}
\bar{\theta}^{2 i} \\
\bar{\theta}^{1 i}
\end{array}\right), \quad \tau_{1}\left(\begin{array}{c}
\eta_{i}^{\mathrm{i}} \\
\eta_{i}^{\dot{2}}
\end{array}\right)=\left(\begin{array}{c}
\bar{\eta}_{i}^{\dot{2}} \\
\bar{\eta}_{i}^{\mathrm{i}}
\end{array}\right)
$$

and

$$
\tau_{0}\left(\theta^{\alpha i}\right)=\bar{\theta}^{\alpha i} \quad \text { and } \quad \tau_{0}\left(\eta_{i}^{\dot{\alpha}}\right)=\bar{\eta}_{i}^{\dot{\alpha}}
$$

which matches the definition for commuting spinors.

In the Euclidean case $\varepsilon=-1$, we can only fix a real structure on the fermionic coordinates if the number of supersymmetries $\mathcal{N}$ is even (see e.g., $[48,49])$. In these cases, one groups together the fermionic coordinates in pairs of two and defines matrices

$$
\left(\epsilon_{s}^{r}\right):=\left(\begin{array}{cc}
0 & -1 \\
1 & 0
\end{array}\right), r, s=1,2 \quad \text { and } \quad\left(T_{j}^{i}\right):=\left(\begin{array}{cc}
\epsilon & 0 \\
0 & \epsilon
\end{array}\right), i, j=1, \ldots, 4 .
$$

The action of $\tau_{-1}$ is then given by

$$
\tau_{-1}\left(\begin{array}{ll}
\theta^{11} & \theta^{12} \\
\theta^{21} & \theta^{22}
\end{array}\right)=\left(\begin{array}{cc}
0 & -1 \\
1 & 0
\end{array}\right)\left(\begin{array}{cc}
\bar{\theta}^{11} & \bar{\theta}^{12} \\
\bar{\theta}^{21} & \bar{\theta}^{22}
\end{array}\right)\left(\begin{array}{cc}
0 & -1 \\
1 & 0
\end{array}\right)
$$

for $\mathcal{N}=2$ and

$$
\tau_{-1}\left(\begin{array}{ccc}
\theta^{11} & \ldots & \theta^{14} \\
\theta^{21} & \cdots & \theta^{24}
\end{array}\right)=\left(\begin{array}{cc}
0 & -1 \\
1 & 0
\end{array}\right)\left(\begin{array}{ccc}
\bar{\theta}^{11} & \ldots & \bar{\theta}^{14} \\
\bar{\theta}^{21} & \ldots & \bar{\theta}^{24}
\end{array}\right)\left(\begin{array}{cccc}
0 & -1 & 0 & 0 \\
1 & 0 & 0 & 0 \\
0 & 0 & 0 & -1 \\
0 & 0 & 1 & 0
\end{array}\right)
$$

for $\mathcal{N}=4$. The last equation can also be written in components as

$$
\tau_{-1}\left(\theta^{\alpha i}\right)=-\varepsilon^{\alpha \beta} T_{j}^{i} \bar{\theta}^{\beta j},
$$

\footnotetext{
${ }^{22}$ For the definition of $\lambda_{ \pm}^{\dot{\alpha}}$, see Section 2 .
} 
where there is a summation over $\beta$ and $j$. The same definition applies to $\eta_{i}^{\dot{\alpha}}$ :

$$
\tau_{-1}\left(\eta_{i}^{\dot{\alpha}}\right)=-\varepsilon^{\dot{\alpha} \dot{\beta}} T_{i}^{j} \bar{\eta}_{j}^{\dot{\beta}}
$$

In general, we define furthermore $\tau\left(\xi^{1} \xi^{2}\right)=\tau\left(\xi^{1}\right) \tau\left(\xi^{2}\right)$ for the product of any two Graßmann variables. ${ }^{23}$ These conventions imply

$$
\overline{\frac{\partial}{\partial \xi}}=\frac{\partial}{\partial \bar{\xi}}
$$

for any Graßmann variable $\xi$.

Using the involutions (4.21) and (4.22), we can impose the following Majorana conditions on the odd variables $\theta^{\alpha i}$ and $\eta_{i}^{\dot{\alpha}}$

$$
\begin{aligned}
& \tau_{1}\left(\theta^{\alpha i}\right)=\theta^{\alpha i} \quad \text { and } \quad \tau_{1}\left(\eta_{i}^{\dot{\alpha}}\right)=\eta_{i}^{\dot{\alpha}} \Longleftrightarrow \theta^{2 i}=\bar{\theta}^{1 i} \quad \text { and } \quad \eta_{i}^{\dot{2}}=\bar{\eta}_{i}^{\dot{1}}, \\
& \tau_{0}\left(\theta^{\alpha i}\right)=\theta^{\alpha i} \quad \text { and } \quad \tau_{0}\left(\eta_{i}^{\dot{\alpha}}\right)=\eta_{i}^{\dot{\alpha}} \Longleftrightarrow \theta^{\alpha i}=\bar{\theta}^{\alpha i} \quad \text { and } \quad \eta_{i}^{\dot{\alpha}}=\bar{\eta}_{i}^{\dot{\alpha}} .
\end{aligned}
$$

For the Euclidean case (4.24)-(4.27), the reality conditions

$$
\tau_{-1}\left(\theta^{\alpha i}\right)=\theta^{\alpha i} \quad \text { and } \quad \tau_{-1}\left(\eta_{i}^{\dot{\alpha}}\right)=\eta_{i}^{\dot{\alpha}}
$$

can be read off from (4.24) and (4.25) for $\mathcal{N}=2$ and $\mathcal{N}=4$, respectively. For instance, for $\mathcal{N}=2$, we have

$$
\theta^{22}=-\bar{\theta}^{11} \text { and } \theta^{21}=\bar{\theta}^{12} .
$$

Imposing the reality conditions $(2.16)$ or $(2.17)$ on $x^{\alpha \dot{\alpha}}$ and corresponding conditions from (4.29)-(4.32) on $\theta^{\alpha i}$ and $\eta_{i}^{\dot{\alpha}}$, we obtain real chiral coordinates $x_{\mathrm{L}}^{\alpha \dot{\alpha}}$ and $x_{\mathrm{R}}^{\alpha \dot{\alpha}}$ for all three real structures. Note that contrary to the Minkowskian signature $(3,1)$, the variables $\theta^{\alpha i}$ and $\eta_{i}^{\dot{\alpha}}$ are independent for both signatures $(4,0)$ and $(2,2)$.

We concentrate now on the real cases defined by the involutions $\tau_{1}$ and $\tau_{-1}$. Note in advance that complexified self-dual $\mathcal{N}$-extended SYM theory can be described by the diagram (4.6) with coordinates $\left(x_{\mathrm{R}}^{\alpha \dot{\alpha}}, \eta_{i}^{\dot{\alpha}}\right)$ on $\mathcal{M}_{\mathrm{R}}^{4 \mid 2 \mathcal{N}}$ and $\left(z_{ \pm}^{\alpha}, \lambda_{\dot{\alpha}}^{ \pm}, \eta_{i}^{ \pm}\right)=\left(x_{\mathrm{R}}^{\alpha \dot{\alpha}} \lambda_{\dot{\alpha}}^{ \pm}, \lambda_{\dot{\alpha}}^{ \pm}, \eta_{i}^{\dot{\alpha}} \lambda_{\dot{\alpha}}^{ \pm}\right)$on $\mathcal{P}^{3 \mid \mathcal{N}}$. After imposing the reality condition (2.16) and (4.29) or (4.31), the coordinates $\left(x_{\mathrm{R}}^{\alpha \dot{\alpha}}, \eta_{i}^{\dot{\alpha}}\right)$ belong to the real antichiral superspace $\mathbb{R}^{4 \mid 2 \mathcal{N}}=: \mathcal{R}_{\mathrm{R}}^{4 \mid 2 \mathcal{N}}$ of real dimension ${ }^{24}(4 \mid 2 \mathcal{N})$. We keep the coordinates $\lambda_{\dot{\alpha}}^{ \pm}$complex $^{25}$ and therefore the supertwistor space $\mathcal{P}^{3 \mid \mathcal{N}}$ has complex dimension $(3 \mid \mathcal{N})$.

\footnotetext{
${ }^{23}$ See Appendix B.

${ }^{24}$ Note that in Minkowski signature, chiral and antichiral superspaces are always complex.

${ }^{25}$ In the Euclidean case, there are no fixed points of $\tau_{-1}$ on the Riemann sphere $\mathbb{C} P^{1} \ni$ $\left(\lambda_{\dot{\alpha}}^{ \pm}\right)$and therefore $\lambda_{\dot{\alpha}}^{ \pm}$must be complex. The case of signature $(2,2)$ is discussed in Appendices C and D.
} 
For coordinates $x_{\mathrm{R}}^{\alpha \dot{\alpha}}$ satisfying (2.16), the vector fields (4.19) and

$$
\bar{V}_{3}^{ \pm}=\partial_{\bar{\lambda}_{ \pm}}
$$

can be identified with bosonic vector fields of type $(0,1)$ on $\mathcal{P}^{3 \mid \mathcal{N}}$ similar to the vector fields $(2.25)$ and $(2.26)$ on $\mathcal{P}^{3 \mid 0}$. In the Euclidean case, this is due to the fact that as a real supermanifold, $\mathcal{P}^{3 \mid \mathcal{N}}$ is diffeomorphic ${ }^{26}$ to the space $\mathcal{R}_{\mathrm{R}}^{4 \mid 2 \mathcal{N}} \times \mathbb{C} P^{1}$,

$$
\mathcal{P}^{3 \mid \mathcal{N}} \cong \mathcal{R}_{\mathrm{R}}^{4 \mid 2 \mathcal{N}} \times \mathbb{C} P^{1}=\pi_{1}^{-1}\left(\mathcal{R}_{\mathrm{R}}^{4 \mid 2 \mathcal{N}}\right) \subset \mathcal{F}_{\mathrm{R}}^{5 \mid 2 \mathcal{N}},
$$

where $\pi_{1}$ is one of the projections in the diagram (4.6). In other words, the map $\pi_{2}$, restricted to $\pi_{1}^{-1}\left(\mathcal{R}_{\mathrm{R}}^{4 \mid 2 \mathcal{N}}\right)$, is one-to-one (cf. (2.19) for the purely bosonic case). Moreover, (4.20) become odd vector fields of type $(0,1)$ on $\mathcal{P}^{3 \mid \mathcal{N}}$ annihilating all complex coordinates on this space. For example, for $\tau_{1}$-real vector fields with $\left|\lambda_{ \pm}\right| \neq 1$, we have

$$
\begin{aligned}
& \bar{\partial}_{+}^{i}=\lambda_{+}^{\dot{\alpha}} \frac{\partial}{\partial \eta_{i}^{\dot{\alpha}}}=\frac{\partial}{\partial \bar{\eta}_{i}^{\dot{1}}}-\lambda_{+} \frac{\partial}{\partial \eta_{i}^{\dot{1}}}, \\
& \bar{\partial}_{+}^{i} \eta_{j}^{+}=0 \text { and } \bar{\partial}_{+}^{i} \bar{\eta}_{j}^{+}=\gamma_{+}^{-1} \delta_{j}^{i},
\end{aligned}
$$

where $\eta_{i}^{+}=\eta_{i}^{\dot{1}}+\lambda_{+} \bar{\eta}_{i}^{\dot{i}}$ and $\gamma_{+}$is given in (2.24). Similar formulæ can be written down for $\tau_{-1}$-real and $\tau_{0}$-real cases. Thus, in the real setup, the double fibration (4.6) simplifies to the nonholomorphic fibration

$$
\pi: \mathcal{P}^{3 \mid \mathcal{N}} \longrightarrow \mathcal{R}_{\mathrm{R}}^{4 \mid 2 \mathcal{N}}
$$

where $(3 \mid \mathcal{N})$ stands for complex and $(4 \mid 2 \mathcal{N})$ for real dimensions. Fibres over a point $\left(x_{\mathrm{R}}, \eta\right) \in \mathcal{R}_{\mathrm{R}}^{4 \mid 2 \mathcal{N}}$ in the fibration (4.37) are real holomorphic sections $\mathbb{C} P_{x_{\mathrm{R}}, \eta}^{1} \hookrightarrow \mathcal{P}^{3 \mid \mathcal{N}}$ described by $(4.5)$ with real $\left(x_{\mathrm{R}}, \eta\right) \in \mathcal{R}_{\mathrm{R}}^{4 \mid 2 \mathcal{N}}$.

\section{$5 \quad$ Supertwistor description of self-dual $\mathcal{N}$-extended SYM theory}

\subsection{Super-self-duality for extended supersymmmetry}

SDYM fields on $\mathbb{R}^{4,0}$ and $\mathbb{R}^{2,2}$ are solutions to the self-duality equations

$$
F_{\mu \nu}=\frac{1}{2} \varepsilon_{\mu \nu \rho \sigma} F^{\rho \sigma} \quad \text { or } \quad F=* F,
$$

\footnotetext{
${ }^{26}$ In the case $\varepsilon=+1$ (and also for the real structure $\tau_{0}$ ), there is a diffeomorphism of an open subset $\tilde{\mathcal{P}}^{3 \mid \mathcal{N}}$ of $\mathcal{P}^{3 \mid \mathcal{N}}$ onto the space $\mathcal{R}_{\mathrm{R}}^{4 \mid 2 \mathcal{N}} \times\left(\mathbb{C} P^{1} \backslash S^{1}\right)$. See Appendix C for more details.
} 
which is equivalently written in spinor notation as

$$
f_{\dot{\alpha} \dot{\beta}}:=-\frac{1}{2} \varepsilon^{\alpha \beta}\left(\partial_{\alpha \dot{\alpha}} A_{\beta \dot{\beta}}-\partial_{\beta \dot{\beta}} A_{\alpha \dot{\alpha}}+\left[A_{\alpha \dot{\alpha}}, A_{\beta \dot{\beta}}\right]\right)=0 .
$$

Solutions to these equations form a subset of the solution space of YangMills theory. Thus, a possible supersymmetric extension of the self-duality equations can be obtained by taking the full set of SYM field equations and imposing certain constraints on them. These constraints have to include (5.1) and keep the resulting set of equations invariant under supersymmetry transformations. This works for SYM theories with $\mathcal{N} \leq 3$, and the field content of the full $\mathcal{N}$-extended SYM theory splits into a self-dual supermultiplet and an anti-self-dual supermultiplet. For $\mathcal{N}=4$, the situation is more complicated, as the SYM multiplet $\left(f_{\alpha \beta}, \chi^{\alpha i}, \phi^{i j}, \tilde{\chi}_{\dot{\alpha} i}, f_{\dot{\alpha} \dot{\beta}}\right)$, where the fields have the helicities $(+1,+1 / 2,0,-1 / 2,-1)$, is irreducible. By introducing an additional field $G_{\dot{\alpha} \dot{\beta}}$ with helicity -1 , which takes in some sense the place of $f_{\dot{\alpha} \dot{\beta}}$, one can circumvent this problem (see e.g., [35,40]). Using this field, one can even obtain the $\mathcal{N}=4$ super-SDYM equations of motion from an action principle [35]. Note that although the field content appearing in the corresponding Lagrangian is $\left(f_{\alpha \beta}, \chi^{\alpha i}, \phi^{i j}, \tilde{\chi}_{\dot{\alpha} i}, f_{\dot{\alpha} \dot{\beta}}, G_{\dot{\alpha} \dot{\beta}}\right), f_{\dot{\alpha} \dot{\beta}}$ vanishes due to the SDYM equations of motion and the supermultiplet of nontrivial fields is $\left(f_{\alpha \beta}, \chi^{\alpha i}, \phi^{i j}, \tilde{\chi}_{\dot{\alpha} i}, G_{\dot{\alpha} \dot{\beta}}\right)$. These degrees of freedom match exactly those of the full $\mathcal{N}=4 \mathrm{SYM}$ and often - by a slight abuse of language - it is stated that they are the same. Following this line, one can even consider the full $\mathcal{N}=4$ SYM theory and $\mathcal{N}=4$ SDYM as the same theories on linearized level, which are only distinguished by different interactions.

For all values $0 \leq \mathcal{N} \leq 4$, supersymmetrically extended self-dual SYM theories can be nicely described in terms of twistor theory, which will be shown in this section.

\subsection{Holomorphic bundles and gauge potentials}

The twistor description of complexified self-dual $\mathcal{N}$-extended SYM theory is known and based on the diagram (4.12) (see e.g., [29,30,32]) or (implicitly) on the diagram (4.6) (see e.g., $[37,40]$ ). Here, we consider self-dual $\mathcal{N}$-extended SYM theory in the real setting based on the fibration (4.37). Namely, let us consider a holomorphic bundle $\mathcal{E}$ over the supertwistor space $\mathcal{P}^{3 \mid \mathcal{N}}=\hat{\mathcal{U}}_{+} \cup \hat{\mathcal{U}}_{-}$without $\mathbb{Z}_{2}$-grading on the fibres and with the coordinates (2.1) and (4.1). As usual, the bundle $\mathcal{E} \rightarrow \mathcal{P}^{3 \mid \mathcal{N}}$ is defined by a holomorphic transition function annihilated by the vector fields (4.19), (4.20) and (4.33) 
of type $(0,1)$ on $\mathcal{P}^{3 \mid \mathcal{N}}$,

$$
\begin{gathered}
\bar{V}_{\alpha}^{+} f_{+-}=0 \\
\partial_{\bar{\lambda}_{+}} f_{+-}=0
\end{gathered}
$$

and

$$
\bar{\partial}_{+}^{i} f_{+-}=0
$$

Further, it is assumed that the restriction of $\mathcal{E}$ to any projective line $\mathbb{C} P_{x_{\mathrm{R}}, \eta}^{1} \hookrightarrow \mathcal{P}^{3 \mid \mathcal{N}}$ is holomorphically trivial and therefore there exist regular matrix-valued functions $\psi_{+}\left(x_{\mathrm{R}}^{\alpha \dot{\alpha}}, \lambda_{+}, \eta_{i}^{\dot{\alpha}}\right)$ on $\hat{\mathcal{U}}_{+}$and $\psi_{-}\left(x_{\mathrm{R}}^{\alpha \dot{\alpha}}, \lambda_{-}, \eta_{i}^{\dot{\alpha}}\right)$ on $\hat{\mathcal{U}}_{-}$ such that

$$
f_{+-}=\psi_{+}^{-1} \psi_{-}
$$

and

$$
\partial_{\bar{\lambda}_{ \pm}} \psi_{ \pm}=0
$$

This trivialization is similar to (3.2) and (3.3) in the purely bosonic case, and using arguments identical to those from Section 3, one can introduce matrix-valued components of a gauge potential,

$$
\begin{aligned}
\mathcal{A}_{\alpha}^{+} & \left.:=\bar{V}_{\alpha}^{+}\right\lrcorner \mathcal{A}=\psi_{+} \bar{V}_{\alpha}^{+} \psi_{+}^{-1}=\psi_{-} \bar{V}_{\alpha}^{+} \psi_{-}^{-1}=\lambda_{+}^{\dot{\alpha}} \mathcal{A}_{\alpha \dot{\alpha}}\left(x_{\mathrm{R}}, \eta\right), \\
\mathcal{A}_{\bar{\lambda}_{+}} & \left.:=\partial_{\bar{\lambda}_{+}}\right\lrcorner \mathcal{A}=\psi_{+} \partial_{\bar{\lambda}_{+}} \psi_{+}^{-1}=\psi_{-} \partial_{\bar{\lambda}_{+}} \psi_{-}^{-1}=0, \\
\mathcal{A}_{+}^{i} & \left.:=\bar{\partial}_{+}^{i}\right\lrcorner \mathcal{A}=\psi_{+} \bar{\partial}_{+}^{i} \psi_{+}^{-1}=\psi_{-} \bar{\partial}_{+}^{i} \psi_{-}^{-1}=\lambda_{+}^{\dot{\alpha}} \mathcal{A}_{\dot{\alpha}}^{i}\left(x_{\mathrm{R}}, \eta\right),
\end{aligned}
$$

where $\left(x_{\mathrm{R}}, \eta\right)=\left(x_{\mathrm{R}}^{\alpha \dot{\alpha}}, \eta_{i}^{\dot{\alpha}}\right)$, and a linear system

$$
\begin{aligned}
\left(\bar{V}_{\alpha}^{+}+\mathcal{A}_{\alpha}^{+}\right) \psi_{+} & =0, \\
\partial_{\bar{\lambda}_{+}} \psi_{+} & =0, \\
\left(\bar{\partial}_{+}^{i}+\mathcal{A}_{+}^{i}\right) \psi_{+} & =0 .
\end{aligned}
$$

of differential equations equivalent to the existence of holomorphic sections of the bundle $\mathcal{E}$.

\subsection{Super-self-duality}

The compatibility conditions of the linear system (5.11)-(5.13) read

$$
\begin{aligned}
{\left[\nabla_{\alpha \dot{\alpha}}, \nabla_{\beta \dot{\beta}}\right]+\left[\nabla_{\alpha \dot{\beta}}, \nabla_{\beta \dot{\alpha}}\right] } & =0, \quad\left[\nabla_{\dot{\alpha}}^{i}, \nabla_{\beta \dot{\beta}}\right]+\left[\nabla_{\dot{\beta}}^{i}, \nabla_{\beta \dot{\alpha}}\right]=0, \\
\left\{\nabla_{\dot{\alpha}}^{i}, \nabla_{\dot{\beta}}^{j}\right\}+\left\{\nabla_{\dot{\beta}}^{i}, \nabla_{\dot{\alpha}}^{j}\right\} & =0,
\end{aligned}
$$

where we have introduced covariant derivatives

$$
\nabla_{\alpha \dot{\alpha}}:=\partial_{\alpha \dot{\alpha}}^{R}+\mathcal{A}_{\alpha \dot{\alpha}} \quad \text { and } \quad \nabla_{\dot{\alpha}}^{i}:=\partial_{\dot{\alpha}}^{i}+\mathcal{A}_{\dot{\alpha}}^{i} .
$$


The compatibility conditions (5.14) suggest the introduction of the following self-dual super gauge field strengths

$$
\begin{aligned}
{\left[\nabla_{\alpha \dot{\alpha}}, \nabla_{\beta \dot{\beta}}\right] } & =\varepsilon_{\dot{\alpha} \dot{\beta}} f_{\alpha \beta}\left(x_{\mathrm{R}}, \eta\right), \quad\left[\nabla_{\dot{\alpha}}^{i}, \nabla_{\beta \dot{\beta}}\right]=\varepsilon_{\dot{\alpha} \dot{\beta}} f_{\beta}^{i}\left(x_{\mathrm{R}}, \eta\right), \\
\left\{\nabla_{\dot{\alpha}}^{i}, \nabla_{\dot{\beta}}^{j}\right\} & =\varepsilon_{\dot{\alpha} \dot{\beta}} f^{i j}\left(x_{\mathrm{R}}, \eta\right),
\end{aligned}
$$

where $f^{i j}$ is antisymmetric and $f_{\alpha \beta}$ is symmetric.

Let us focus on the case $\mathcal{N}=4$ and discuss the cases $\mathcal{N}<4$ later on. The set of physical fields for $\mathcal{N}=4$ SYM theory consists of the self-dual and the anti-self-dual field strengths of a gauge potential $\mathcal{A}_{\alpha \dot{\alpha}}$, four spinors $\chi_{\alpha}^{i}$ together with four spinors $\tilde{\chi}_{\dot{\alpha} i} \sim \varepsilon_{i j k l} \tilde{\chi}_{\dot{\alpha}}^{j k l}$ of opposite chirality and six real (or three complex) scalars $\phi^{i j}=\phi^{[i j]}$. For $\mathcal{N}=4$ super-SDYM, the multiplet is joined by an additional spin-one field $G_{\dot{\alpha} \dot{\beta}} \sim \varepsilon_{i j k l} G_{\dot{\alpha} \dot{\beta}}^{i j k l}$, as discussed before. Now the above super gauge field strengths contain in their expansion exactly these fields. The lowest component of $f_{\alpha \beta}, f_{\alpha}^{i}$ and $f^{i j}$ will be the SDYM field strength, the spinor field $\chi_{\alpha}^{i}$ and the scalars $\phi^{i j}$, respectively. By using Bianchi identities for the self-dual super gauge field strengths, one obtains [40] successively the superfield expansions and the field equations for the physical field content, ${ }^{27}$

$$
\begin{aligned}
& f_{\dot{\alpha} \dot{\beta}}=-\frac{1}{2} \varepsilon^{\alpha \beta}\left(\partial_{\alpha \dot{\alpha}} A_{\beta \dot{\beta}}-\partial_{\beta \dot{\beta}} A_{\alpha \dot{\alpha}}+\left[A_{\alpha \dot{\alpha}}, A_{\beta \dot{\beta}}\right]\right)=0, \\
& \nabla_{\alpha \dot{\alpha}} \chi^{\alpha i}=0, \\
& \square \phi^{i j}+2 \varepsilon\left\{\chi^{\alpha i}, \chi_{\alpha}^{j}\right\}=0, \\
& \nabla_{\alpha \dot{\alpha}} \tilde{\chi}^{\dot{\alpha} i j k}-\varepsilon\left[\chi_{\alpha}^{[i}, \phi^{j k]}\right]=0, \\
& \varepsilon^{\dot{\alpha} \dot{\gamma}} \nabla_{\alpha \dot{\alpha}} G_{\dot{\gamma} \dot{\delta}}^{[i j k l]}+12 \varepsilon\left\{\chi_{\alpha}^{[i}, \tilde{\chi}_{\dot{\delta}}^{j k l]}\right\}-18 \varepsilon\left[\phi^{[i j}, \nabla_{\alpha \dot{\delta}} \phi^{k l]}\right]=0,
\end{aligned}
$$

where $\square:=\nabla_{\alpha \dot{\alpha}} \nabla^{\alpha \dot{\alpha}}$ and the antisymmetrizations $[i \cdots j]$ are defined to have weight $^{28}$ one. Note that by construction, all fields depend on the coordinates $x_{\mathrm{R}}^{\alpha \dot{\alpha}}$. As we will see soon, (5.17) is in some sense an $\mathcal{N}$-independent formulation [40] of the field equations of super-SDYM theory in which the cases $\mathcal{N}<4$ are governed by the first $\mathcal{N}+1$ equations of (5.17), where $f_{\dot{\alpha} \dot{\beta}}=0$ is counted as one equation and so on. In the case $\mathcal{N}=4$, one can introduce

\footnotetext{
${ }^{27}$ The fields are scaled to match the discussion following (5.33) and (5.34).

${ }^{28}$ So $[i j k]:=i j k+j k i+k i j-k j i-j i k-i k j$ and $[i j k l]$ is obtained by contracting with $\varepsilon_{i j k l}$.
} 
"dualized" fields

$$
\phi_{i j}:=\frac{1}{2 !} \varepsilon_{i j k l} \phi^{k l}, \quad \tilde{\chi}_{i}^{\dot{\alpha}}:=\frac{1}{3 !} \varepsilon_{i j k l} \tilde{\chi}^{\dot{\alpha} j k l} \quad \text { and } \quad G_{\dot{\alpha} \dot{\beta}}:=\frac{1}{4 !} \varepsilon_{i j k l} G_{\dot{\alpha} \dot{\beta}}^{i j k l},
$$

for which the equations of motion take the form

$$
\begin{aligned}
& f_{\dot{\alpha} \dot{\beta}}=0, \\
& \nabla_{\alpha \dot{\alpha}} \chi^{\alpha i}=0, \\
& \square \phi^{i j}+2 \varepsilon\left\{\chi^{\alpha i}, \chi_{\alpha}^{j}\right\}=0, \\
& \nabla_{\alpha \dot{\alpha}} \tilde{\chi}_{i}^{\dot{\alpha}}-2 \varepsilon\left[\chi_{\alpha}^{j}, \phi_{i j}\right]=0, \\
& \varepsilon^{\dot{\alpha} \dot{\gamma}} \nabla_{\alpha \dot{\alpha}} G_{\dot{\gamma} \dot{\delta}}+3 \varepsilon\left\{\chi_{\alpha}^{i}, \tilde{\chi}_{\dot{\delta} i}\right\}-\frac{3}{2} \varepsilon\left[\phi_{i j}, \nabla_{\alpha \dot{\delta}} \phi^{i j}\right]=0 .
\end{aligned}
$$

After rescaling some of the fields as

$$
\chi_{\alpha}^{i} \longrightarrow \frac{1}{2} \chi_{\alpha}^{i}, \quad \phi^{i j} \longrightarrow \frac{1}{2} \phi^{i j}, \quad \tilde{\chi}_{\dot{\alpha} i} \longrightarrow-\frac{1}{2} \tilde{\chi}_{\dot{\alpha} i}, \quad G_{\dot{\alpha} \dot{\beta}} \longrightarrow \frac{3}{2} G_{\dot{\alpha} \dot{\beta}},
$$

the equations (5.19) are the field equations of the Lagrangian for $\mathcal{N}=4$ self-dual SYM given in [2].

\subsection{Gauge equivalent trivializations}

We have described the twistor correspondence between holomorphic bundles $\mathcal{E}$ over the supertwistor space $\mathcal{P}^{3 \mid \mathcal{N}}$ trivial on projective lines in $\mathcal{P}^{3 \mid \mathcal{N}}$ and solutions to the field equations of self-dual $\mathcal{N}$-extended SYM theory on the space $\mathbb{R}^{4}$ with metric $\left(g_{\mu \nu}\right)=\operatorname{diag}(-\varepsilon,-\varepsilon,+1,+1)$. The derivation of the $\mathcal{N}=4$ super-self-duality equations (5.17) is based on trivializations of $\mathcal{E}$ over $\hat{\mathcal{U}}_{ \pm}$such that equations (5.7) and therefore (5.9) are satisfied. However, there are other convenient trivializations of $\mathcal{E}$ over $\hat{\mathcal{U}}_{ \pm}$such that the compatibility conditions of the corresponding linear system are described by hCS theory $[1,2]$ on the supertwistor space. Namely, since restrictions of the bundle $\mathcal{E}$ to $(2 \mid \mathcal{N})$-dimensional leaves of the fibration $(4.3)$ are trivial, ${ }^{29}$ there exist $\tau_{\varepsilon}$-regular ${ }^{30}$ matrix-valued functions $\hat{\psi}_{ \pm}$on $\tilde{\mathcal{U}}_{ \pm}$such that

$$
f_{+-}=\psi_{+}^{-1} \psi_{-}=\hat{\psi}_{+}^{-1} \hat{\psi}_{-}
$$

and

$$
\bar{\partial}_{ \pm}^{i} \hat{\psi}_{ \pm}=0
$$

This implies $\hat{\psi}_{ \pm}=\hat{\psi}_{ \pm}\left(x_{\mathrm{R}}^{\alpha \dot{\alpha}}, \lambda_{ \pm}, \bar{\lambda}_{ \pm}, \eta_{i}^{ \pm}\right)$. These trivializations are analogous to the trivializations $(3.16)-(3.22)$ in the $\mathcal{N}=0$ case. Note that similarly

\footnotetext{
${ }^{29}$ Note that fibres $\mathbb{C}_{\lambda}^{2 \mid \mathcal{N}}$ over $\lambda \in \mathbb{C} P^{1}$ in the fibration $\mathcal{P}^{3 \mid \mathcal{N}} \rightarrow \mathbb{C} P^{1}$ are exactly the $\beta_{\mathrm{R}}$-superplanes introduces in Section 4 . Super-self-duality is equivalent in the discussed superfield formulation to flatness of SYM fields on these $\beta_{\mathrm{R}}$-superplanes.

${ }^{30}$ See the definition on p. 9.
} 
to (3.28) in the purely bosonic case, one can also choose the trivializations $\tilde{\psi}_{ \pm}\left(z_{ \pm}^{\alpha}, \lambda_{ \pm}, \bar{\lambda}_{ \pm}, \eta_{i}^{ \pm}\right)$but we will not consider them here.

\subsection{Linear system}

It follows from (5.21) that

$$
\varphi:=\psi_{+} \hat{\psi}_{+}^{-1}=\psi_{-} \hat{\psi}_{-}^{-1}
$$

is a matrix-valued function (superfield) generating a gauge transformation ${ }^{31}$

$$
\begin{aligned}
& \psi_{ \pm} \longmapsto \hat{\psi}_{ \pm}=\varphi^{-1} \psi_{ \pm}, \\
& \mathcal{A}_{\alpha}^{ \pm} \longmapsto \hat{\mathcal{A}}_{\alpha}^{ \pm}=\varphi^{-1} \mathcal{A}_{\alpha}^{ \pm} \varphi+\varphi^{-1} \bar{V}_{\alpha}^{ \pm} \varphi=\hat{\psi}_{ \pm} \bar{V}_{\alpha}^{ \pm} \hat{\psi}_{ \pm}^{-1}, \\
& 0=\mathcal{A}_{\bar{\lambda}_{ \pm}} \longmapsto \hat{\mathcal{A}}_{\bar{\lambda}_{ \pm}}=\varphi^{-1} \partial_{\bar{\lambda}_{ \pm}} \varphi=\hat{\psi}_{ \pm} \partial_{\bar{\lambda}_{ \pm}} \hat{\psi}_{ \pm}^{-1} \\
& \mathcal{A}_{ \pm}^{i} \longmapsto \hat{\mathcal{A}}_{ \pm}^{i}=\varphi^{-1} \mathcal{A}_{ \pm}^{i} \varphi+\varphi^{-1} \bar{\partial}_{ \pm}^{i} \varphi=\hat{\psi}_{ \pm} \bar{\partial}_{ \pm}^{i} \hat{\psi}_{ \pm}^{-1}=0,
\end{aligned}
$$

where we used (5.7). Thus, we obtain the linear system

$$
\begin{aligned}
\left(\bar{V}_{\alpha}^{+}+\hat{\mathcal{A}}_{\alpha}^{+}\right) \hat{\psi}_{+} & =0 \\
\left(\partial_{\bar{\lambda}_{+}}+\hat{\mathcal{A}}_{\bar{\lambda}_{+}}\right) \hat{\psi}_{+} & =0 \\
\bar{\partial}_{+}^{i} \hat{\psi}_{+} & =0
\end{aligned}
$$

which is gauge equivalent to the linear system (5.11)-(5.13). For $\hat{\psi}_{-}$, we have similar equations which are obtained by changing "+" to "-" in (5.28)(5.30). In the case $\mathcal{N}=0$, this linear system coincides with (3.21) and (3.22).

\subsection{Super-hCS theory}

The compatibility conditions of the linear differential equations (5.28)-(5.30) are the field equations of hCS theory on the supertwistor space ${ }^{32} \mathcal{P}^{3 \mid \mathcal{N}}$. On

\footnotetext{
${ }^{31}$ The function $\varphi$ is $\tau_{\varepsilon}$-regular and, in particular, it can be singular on $\mathcal{P}_{0, \mathcal{N}}:=$ $\left.\mathcal{P}^{3 \mid \mathcal{N}}\right|_{\left|\lambda_{ \pm}\right|=1}$ in the Kleinian case $\varepsilon=+1$, see Appendix D.

${ }^{32}$ More accurately, in the case $\varepsilon=+1$ (and also for the real structure $\tau_{0}$ ), $\hat{\mathcal{A}}^{0,1}$ (and thus hCS theory) is defined only on the subset $\tilde{\mathcal{P}}^{3 \mid \mathcal{N}}$ of $\mathcal{P}^{3 \mid \mathcal{N}}$ for which $\left|\lambda_{ \pm}\right| \neq 1$. See Appendix D for more details.
} 
$\hat{\mathcal{U}}_{+}$they read

$$
\begin{aligned}
\bar{V}_{\alpha}^{+} \hat{\mathcal{A}}_{\beta}^{+}-\bar{V}_{\beta}^{+} \hat{\mathcal{A}}_{\alpha}^{+}+\left[\hat{\mathcal{A}}_{\alpha}^{+}, \hat{\mathcal{A}}_{\beta}^{+}\right]=0, \\
\partial_{\bar{\lambda}_{+}} \hat{\mathcal{A}}_{\alpha}^{+}-\bar{V}_{\alpha}^{+} \hat{\mathcal{A}}_{\bar{\lambda}_{+}}+\left[\hat{\mathcal{A}}_{\bar{\lambda}_{+}}, \hat{\mathcal{A}}_{\alpha}^{+}\right]=0
\end{aligned}
$$

and similarly on $\hat{\mathcal{U}}_{-}$. Here $\hat{\mathcal{A}}_{\alpha}^{+}$and $\hat{\mathcal{A}}_{\bar{\lambda}_{+}}$are functions of $\left(x_{\mathrm{R}}^{\alpha \dot{\alpha}}, \lambda_{+}, \bar{\lambda}_{+}, \eta_{i}^{+}\right)$. These equations are equivalent to the equations of self-dual $\mathcal{N}$-extended SYM theory on $\mathbb{R}^{4}$ which form a subset of equations (5.17). As already mentioned, the most interesting case is $\mathcal{N}=4$ since the supertwistor space $\mathcal{P}^{3 \mid 4}$ is a CY supermanifold and one can derive equations (5.31) and (5.32) from a manifestly Lorentz invariant action $[2,39]$. For this reason, we concentrate on the equivalence with self-dual SYM for the case $\mathcal{N}=4$; for other values of $\mathcal{N}$, the derivation goes along the same lines.

Recall that $\hat{\mathcal{A}}_{\alpha}$ and $\hat{\mathcal{A}}_{\bar{\lambda}}$ are sections of the bundles ${ }^{33} \mathcal{O}(1) \otimes \mathbb{C}^{2}$ and $\overline{\mathcal{O}}(-2)$ over $\mathbb{C} P^{1}$ since the vector fields in $(5.25)$ take values in $\mathcal{O}(1)$ and the holomorphic cotangent bundle of $\mathbb{C} P^{1}$ is $\mathcal{O}(-2)$. Together with the fact that $\eta_{i}^{ \pm}$'s take values in the bundle $\Pi \mathcal{O}(1)$, this fixes the dependence of $\hat{\mathcal{A}}_{\alpha}^{ \pm}$ and $\hat{\mathcal{A}}_{\bar{\lambda}_{ \pm}}$on $\lambda_{ \pm}^{\dot{\alpha}}$ and $\hat{\lambda}_{ \pm}^{\dot{\alpha}}$. In the case $\mathcal{N}=4$, this dependence takes the form

$$
\begin{aligned}
\hat{\mathcal{A}}_{\alpha}^{+}= & \lambda_{+}^{\dot{\alpha}} A_{\alpha \dot{\alpha}}\left(x_{\mathrm{R}}\right)+\eta_{i}^{+} \chi_{\alpha}^{i}\left(x_{\mathrm{R}}\right)+\gamma_{+} \frac{1}{2 !} \eta_{i}^{+} \eta_{j}^{+} \hat{\lambda}_{+}^{\dot{\alpha}} \phi_{\alpha \dot{\alpha}}^{i j}\left(x_{\mathrm{R}}\right) \\
& +\gamma_{+}^{2} \frac{1}{3 !} \eta_{i}^{+} \eta_{j}^{+} \eta_{k}^{+} \hat{\lambda}_{+}^{\dot{\alpha}} \hat{\lambda}_{+}^{\dot{\beta}} \tilde{\chi}_{\alpha \dot{\alpha} \dot{\beta}}^{i j k}\left(x_{\mathrm{R}}\right) \\
& +\gamma_{+}^{3} \frac{1}{4 !} \eta_{i}^{+} \eta_{j}^{+} \eta_{k}^{+} \eta_{l}^{+} \hat{\lambda}_{+}^{\dot{\alpha}} \hat{\lambda}_{+}^{\dot{\beta}} \hat{\lambda}_{+}^{\dot{\gamma}} G_{\alpha \dot{\alpha} \dot{\beta} \dot{\gamma}}^{i j k l}\left(x_{\mathrm{R}}\right), \\
\hat{\mathcal{A}}_{\bar{\lambda}_{+}}= & \gamma_{+}^{2} \frac{1}{2 !} \eta_{i}^{+} \eta_{j}^{+} \phi^{i j}\left(x_{\mathrm{R}}\right)+\gamma_{+}^{3} \frac{1}{3 !} \eta_{i}^{+} \eta_{j}^{+} \eta_{k}^{+} \hat{\lambda}_{+}^{\dot{\alpha}} \tilde{\chi}_{\dot{\alpha}}^{i j k}\left(x_{\mathrm{R}}\right) \\
& +\gamma_{+}^{4} \frac{1}{4 !} \eta_{i}^{+} \eta_{j}^{+} \eta_{k}^{+} \eta_{l}^{+} \hat{\lambda}_{+}^{\dot{\alpha}} \hat{\lambda}_{+}^{\dot{\beta}} G_{\dot{\alpha} \dot{\beta}}^{i j k l}\left(x_{\mathrm{R}}\right),
\end{aligned}
$$

and similar for $\hat{\mathcal{A}}_{\alpha}^{-}, \hat{\mathcal{A}}_{\bar{\lambda}_{-}}$. Here, again, $A_{\alpha \dot{\alpha}}, \chi_{\alpha}^{i}, \phi^{i j}, \tilde{\chi}_{\dot{\alpha} i}$ is the ordinary field content of $\mathcal{N}=4$ SYM theory and the field $G_{\dot{\alpha} \dot{\beta}}$ is the auxiliary field arising in the $\mathcal{N}=4$ self-dual case, as discussed above. It follows from (5.32)-(5.34) that $^{34}$

$$
\phi_{\alpha \dot{\alpha}}^{i j}=-\nabla_{\alpha \dot{\alpha}} \phi^{i j}, \quad \tilde{\chi}_{\alpha \dot{\alpha} \dot{\beta}}^{i j k}=-\frac{1}{4} \nabla_{\alpha(\dot{\alpha}} \tilde{\chi}_{\dot{\beta})}^{i j k} \quad \text { and } \quad G_{\alpha \dot{\alpha} \dot{\beta} \dot{\gamma}}^{i j k l}=-\frac{1}{18} \nabla_{\alpha(\dot{\alpha}} G_{\dot{\beta} \dot{\gamma})}^{i j k l},
$$

\footnotetext{
${ }^{33}$ The bundle $\overline{\mathcal{O}}(n)$ is the complex conjugate to $\mathcal{O}(n)$.

${ }^{34}$ We use the symmetrization (.) with weight one, e.g., $(\dot{\alpha} \dot{\beta})=\dot{\alpha} \dot{\beta}+\dot{\beta} \dot{\alpha}$.
} 
i.e., these fields do not contain additional degrees of freedom. The expansion (5.33), (5.34) together with the field equations (5.31) and (5.32) reproduces exactly equations (5.17).

Consider now the cases $\mathcal{N}<4$. Since the $\eta_{i}^{+}$'s are Graßmann variables and thus nilpotent, the expansion (5.33) and (5.34) will only have terms up to order $\mathcal{N}$ in the $\eta_{i}^{+}$'s. This exactly reduces the expansion to the appropriate field content for $\mathcal{N}$-extended super-SDYM theory

$$
\begin{aligned}
& \mathcal{N}=0 \quad A_{\alpha \dot{\alpha}} \\
& \mathcal{N}=1 \quad A_{\alpha \dot{\alpha}}, \quad \chi_{\alpha}^{i} \quad \text { with } \quad i=1 \\
& \mathcal{N}=2 \quad A_{\alpha \dot{\alpha}}, \quad \chi_{\alpha}^{i}, \quad \phi^{[i j]} \quad \text { with } \quad i, j=1,2 \\
& \mathcal{N}=3 \quad A_{\alpha \dot{\alpha}}, \quad \chi_{\alpha}^{i}, \quad \phi^{[i j]}, \quad \chi_{\dot{\alpha}}^{[i j k]} \quad \text { with } i, j, \ldots=1,2,3 \\
& \mathcal{N}=4 \quad A_{\alpha \dot{\alpha}}, \quad \chi_{\alpha}^{i}, \quad \phi^{[i j]}, \quad \chi_{\dot{\alpha}}^{[i j k]}, \quad G_{\dot{\alpha} \dot{\beta}}^{[i j k l]} \quad \text { with } i, j, \ldots=1, \ldots, 4 .
\end{aligned}
$$

One should note that the antisymmetrization [.] leads to a different number of fields depending on the range of $i$. For example, in the case $\mathcal{N}=2$, there is only one real scalar $\phi^{12}$, while for $\mathcal{N}=4$ there exist six real scalars. Inserting such a truncated expansion for $\mathcal{N}<4$ into the field equations (5.31) and (5.32), we obtain the first $\mathcal{N}+1$ equations of (5.17), which is the appropriate set of equations for $\mathcal{N}<4$ super-SDYM theory.

To sum up, we have described a one-to-one correspondence between gauge equivalence classes of solutions to the $\mathcal{N}$-extended SDYM equations on $\left(\mathbb{R}^{4}, g\right)$ with $g=\operatorname{diag}(-\varepsilon,-\varepsilon,+1,+1)$ and equivalence classes ${ }^{35}$ of holomorphic vector bundles $\mathcal{E}$ over the supertwistor space $\mathcal{P}^{3 \mid \mathcal{N}}$ such that the bundles $\mathcal{E}$ are holomorphically trivial on each projective line $\mathbb{C} P_{x_{\mathrm{R}}, \eta}^{1}$ in $\mathcal{P}^{3 \mid \mathcal{N}}$. In other words, there is a bijection between the moduli spaces of hCS theory on $\mathcal{P}^{3 \mid \mathcal{N}}$ and the one of self-dual $\mathcal{N}$-extended SYM theory on $\left(\mathbb{R}^{4}, g\right)$. It is assumed that appropriate reality conditions are imposed. The PenroseWard transform and its inverse are defined by the formulæ (5.33)-(5.35). In fact, these formulæ relate solutions of the equations of motion of hCS theory on $\mathcal{P}^{3 \mid \mathcal{N}}$ to those of self-dual $\mathcal{N}$-extended SYM theory on $\left(\mathbb{R}^{4}, g\right)$. One can also write integral formulæ of type (3.25) but we refrain from doing this.

\footnotetext{
${ }^{35}$ Two holomorphic bundles $\left(\mathcal{E}, f_{+-}\right)$and $\left(\mathcal{E}^{\prime}, f_{+-}^{\prime}\right)$ over $\mathcal{P}^{3 \mid \mathcal{N}}$ are called equivalent, if there exist regular matrix-valued functions $h_{+}$and $h_{-}$, which are holomorphic on $\hat{\mathcal{U}}_{+}$ and $\hat{\mathcal{U}}_{-}$, respectively, such that the transition functions $f_{+-}$and $f_{+-}^{\prime}$ are related by $f_{+-}^{\prime}=h_{+}^{-1} f_{+-} h_{-}$.
} 


\section{Dual supertwistors and $\mathcal{N}$-extended anti-self-duality}

\subsection{Coordinates}

In Section 4, we described the supertwistor space $\mathbb{C} P^{3 \mid \mathcal{N}}$ as the space of (1|0)-dimensional subspaces in the space $\mathbb{C}^{4 \mid \mathcal{N}}$. Its dual supermanifold can be defined as a space of $(3 \mid \mathcal{N})$-dimensional planes in $\mathbb{C}^{4 \mid \mathcal{N}}$ parametrized by homogeneous coordinates $\left(\mu_{\alpha}, \sigma^{\dot{\alpha}}, \theta^{i}\right)$ subject to the identification $\left(\mu_{\alpha}, \sigma^{\dot{\alpha}}, \theta^{i}\right)$ $\sim\left(t \mu_{\alpha}, t \sigma^{\dot{\alpha}}, t \theta^{i}\right)$ for any nonzero complex number $t$. We again have the supermanifold $^{36} \mathbb{C} P_{*}^{3 \mid \mathcal{N}}$ and the space $\mathcal{P}_{*}^{3 \mid \mathcal{N}}=\mathbb{C} P_{*}^{3 \mid \mathcal{N}} \backslash \mathbb{C} P_{*}^{1 \mid \mathcal{N}}=(\mathcal{O}(1) \oplus$ $\mathcal{O}(1))_{*}^{3 \mid \mathcal{N}}=\hat{\mathcal{V}}_{+} \cup \hat{\mathcal{V}}_{-}$with coordinates

$$
\begin{gathered}
w_{+}^{\dot{\alpha}}=\frac{\sigma^{\dot{\alpha}}}{\mu_{1}}, \quad w_{+}^{\dot{3}}=\zeta_{+}=\frac{\mu_{2}}{\mu_{1}} \quad \text { and } \quad \theta_{+}^{i}=\frac{\theta^{i}}{\mu_{1}} \text { on } \hat{\mathcal{V}}_{+}, \\
w_{-}^{\dot{\alpha}}=\frac{\sigma^{\dot{\alpha}}}{\mu_{2}}, \quad w_{-}^{\dot{3}}=\zeta_{-}=\frac{\mu_{1}}{\mu_{2}} \quad \text { and } \quad \theta_{-}^{i}=\frac{\theta^{i}}{\mu_{2}} \text { on } \hat{\mathcal{V}}_{-}, \\
w_{+}^{\dot{\alpha}}=\zeta_{+} w_{-}^{\dot{\alpha}}, \quad \zeta_{+}=\zeta_{-}^{-1}, \quad \theta_{+}^{i}=\zeta_{+} \theta_{-}^{i} \text { on } \hat{\mathcal{V}}_{+} \cap \hat{\mathcal{V}}_{-} .
\end{gathered}
$$

Note that $\zeta_{ \pm}$are coordinates on the patches $V_{ \pm}=\hat{\mathcal{V}}_{ \pm} \cap \mathbb{C} P_{*}^{1}$ covering the base $^{37} \mathbb{C} P_{*}^{1}=\mathbb{C} P_{*}^{1 \mid 0}$ of the holomorphic vector bundle

$$
\mathcal{P}_{*}^{3 \mid \mathcal{N}} \longrightarrow \mathbb{C} P_{*}^{1 \mid 0}
$$

Sections of this bundle (degree one holomorphic curves $\mathbb{C} P_{x_{\mathrm{L}}, \theta}^{1} \hookrightarrow \mathcal{P}_{*}^{3 \mid \mathcal{N}}$ ) are defined by the equations

$$
w_{ \pm}^{\dot{\alpha}}=x_{\mathrm{L}}^{\alpha \dot{\alpha}} \mu_{\alpha}^{ \pm}, \quad \theta_{ \pm}^{i}=\theta^{\alpha i} \mu_{\alpha}^{ \pm} \quad \text { with } \quad\left(\mu_{\alpha}^{+}\right)=\left(\begin{array}{c}
1 \\
\zeta_{+}
\end{array}\right), \quad\left(\mu_{\alpha}^{-}\right)=\left(\begin{array}{c}
\zeta_{-} \\
1
\end{array}\right)
$$

and parametrized by supermoduli $\left(x_{\mathrm{L}}, \theta\right)=\left(x_{\mathrm{L}}^{\alpha \dot{\alpha}}, \theta^{\alpha i}\right) \in \mathbb{C}^{4 \mid 2 \mathcal{N}}=: \mathcal{M}_{\mathrm{L}}^{4 \mid 2 \mathcal{N}}$. Real sections are obtained by imposing the reality conditions (2.16) or (2.17). We denote the space of such sections by $\mathcal{R}_{\mathrm{L}}^{4 \mid 2 \mathcal{N}}\left(=\mathbb{R}^{4 \mid 2 \mathcal{N}}\right)$.

\footnotetext{
${ }^{36}$ We use the subscript $*$ to denote the dual supertwistor space, its subspaces and its preimages under projections $\pi_{2}$.

${ }^{37}$ Recall that we use the subscript $*$ in $\mathbb{C} P_{*}^{1}$ for distinguishing this Riemann sphere with the coordinates $\zeta_{ \pm}$from the Riemann sphere $\mathbb{C} P^{1}$ with the coordinates $\lambda_{ \pm}$.
} 
Similarly to the supertwistor case, we can introduce a fibration

$$
\mathcal{P}_{*}^{3 \mid \mathcal{N}} \longrightarrow \mathbb{C} P_{*}^{1 \mid \mathcal{N}}
$$

whose holomorphic sections $\mathbb{C} P_{x_{\mathrm{R}}, \eta}^{1 \mid \mathcal{N}} \hookrightarrow \mathcal{P}_{*}^{3 \mid \mathcal{N}}$ are defined by the equations

$$
w_{ \pm}^{\dot{\alpha}}=x_{\mathrm{R}}^{\alpha \dot{\alpha}} \mu_{\alpha}^{ \pm}-2 \eta_{i}^{\dot{\alpha}} \theta_{ \pm}^{i}
$$

and parametrized by supermoduli $\left(x_{\mathrm{R}}, \eta\right)=\left(x_{\mathrm{R}}^{\alpha \dot{\alpha}}, \eta_{i}^{\dot{\alpha}}\right) \in \mathbb{C}^{4 \mid 2 \mathcal{N}}=: \mathcal{M}_{\mathrm{R}}^{4 \mid 2 \mathcal{N}}$. The real subspace $\mathcal{R}_{\mathrm{R}}^{4 \mid 2 \mathcal{N}}$ was considered in Section 4. So for dual supertwistors, we can again introduce double fibrations similar to (4.6) and (4.8) simply by substituting the spaces $\mathcal{F}_{\mathrm{R}}^{5 \mid 2 \mathcal{N}}, \mathcal{F}_{\mathrm{L}}^{5 \mid 3 \mathcal{N}}$ and $\mathcal{P}^{3 \mid \mathcal{N}}$ by the spaces $\mathcal{F}_{\mathrm{R} *}^{5 \mid 2 \mathcal{N}}:=\mathcal{M}_{\mathrm{R}}^{4 \mid 2 \mathcal{N}} \times \mathbb{C} P_{*}^{1}, \quad \mathcal{F}_{\mathrm{L} *}^{5 \mid 3 \mathcal{N}}:=\mathcal{M}_{\mathrm{L}}^{4 \mid 2 \mathcal{N}} \times \mathbb{C} P_{*}^{1 \mid \mathcal{N}} \quad$ and $\quad \mathcal{P}_{*}^{3 \mid \mathcal{N}} \supset \mathbb{C} P_{*}^{1}$, respectively.

From (6.5) and (6.7), we obtain again formulæ (4.9), (4.10) and the equations

$$
w_{ \pm}^{\dot{\alpha}}=\left(x^{\alpha \dot{\alpha}}+\theta^{\alpha i} \eta_{i}^{\dot{\alpha}}\right) \mu_{\alpha}^{ \pm} \quad \text { and } \quad \theta_{ \pm}^{i}=\theta^{\alpha i} \mu_{\alpha}^{ \pm}
$$

defining projective lines $\mathbb{C} P_{x, \theta, \eta}^{1} \hookrightarrow \mathcal{P}_{*}^{3 \mid \mathcal{N}}$ parametrized by supermoduli $(x, \theta, \eta)=\left(x^{\alpha \dot{\alpha}}, \theta^{\alpha i}, \eta_{i}^{\dot{\alpha}}\right) \in \mathbb{C}^{4 \mid 4 \mathcal{N}}$. Via the dual supertwistor correspondence, a point $p=\left(w_{ \pm}^{\dot{\alpha}}, \zeta_{ \pm}, \theta_{ \pm}^{i}\right) \in \mathcal{P}_{*}^{3 \mid \mathcal{N}}$ corresponds to a totally null $\alpha_{\mathrm{L}^{-}}, \alpha_{\mathrm{R}^{-}}$or $\alpha$-superplane in $\mathcal{M}_{\mathrm{L}}^{4 \mid 2 \mathcal{N}}, \mathcal{M}_{\mathrm{R}}^{4 \mid 2 \mathcal{N}}$ or $\mathbb{C}^{4 \mid 4 \mathcal{N}}$ with dimension $(2 \mid \mathcal{N}),(2 \mid 2 \mathcal{N})$ or $(2 \mid 3 \mathcal{N})$, respectively.

\subsection{Vector fields}

Using (6.8) and (6.5), one can introduce a double fibration

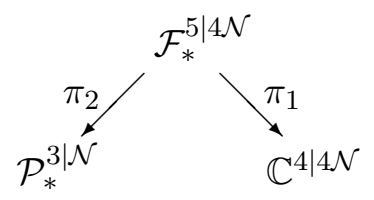

for the complex nonchiral case and the fibration

$$
\pi: \mathcal{P}_{*}^{3 \mid \mathcal{N}} \longrightarrow \mathcal{R}_{\mathrm{L}}^{4 \mid 2 \mathcal{N}}
$$

in the case of $\left(x^{\alpha \dot{\alpha}}\right)$ and $\left(\theta^{\alpha i}, \eta_{i}^{\dot{\alpha}}\right)$ satisfying the reality conditions induced by $\tau_{\varepsilon}$ as discussed in Section 4 . The superspace $\mathbb{C}^{4 \mid 4 \mathcal{N}}$ and its real subspace $\mathcal{R}_{\mathrm{L}}^{4 \mid 2 \mathcal{N}}$ are the same as in Section 4 and parametrized by the same 
coordinates. We have coordinates

$$
\begin{aligned}
& \left(x^{\alpha \dot{\alpha}}, \mu_{\alpha}^{ \pm}, \theta^{\alpha i}, \eta_{i}^{\dot{\alpha}}\right) \quad \text { on } \mathcal{F}_{*}^{5 \mid 4 \mathcal{N}} \\
& \left(x_{\mathrm{L}}^{\alpha \dot{\alpha}} \mu_{\alpha}^{ \pm}, \mu_{\alpha}^{ \pm}, \theta^{\alpha i} \mu_{\alpha}^{ \pm}\right) \quad \text { on } \mathcal{P}_{*}^{3 \mid \mathcal{N}}
\end{aligned}
$$

with obvious projections in the fibrations (6.9) and (6.10).

It is not difficult to see that the tangent spaces of the $(2 \mid 3 \mathcal{N})$-dimensional leaves of the projection $\pi_{2}: \mathcal{F}_{*}^{5 \mid 4 \mathcal{N}} \rightarrow \mathcal{P}_{*}^{3 \mid \mathcal{N}}$ from (6.9) are spanned by the vector fields

$$
\begin{gathered}
D_{\dot{\alpha}}^{i}=\partial_{\dot{\alpha}}^{i}+\theta^{\alpha i} \partial_{\alpha \dot{\alpha}}, \\
D_{i}^{ \pm}=\mu_{ \pm}^{\alpha} D_{\alpha i} \quad \text { with } D_{\alpha i}=\partial_{\alpha i}+\eta_{i}^{\dot{\alpha}} \partial_{\alpha \dot{\alpha}}, \quad\left(\mu_{+}^{\alpha}\right)=\left(\begin{array}{c}
-\zeta_{+} \\
1
\end{array}\right), \quad\left(\mu_{-}^{\alpha}\right)=\left(\begin{array}{c}
-1 \\
\zeta_{-}
\end{array}\right),
\end{gathered}
$$

and

$$
\bar{V}_{\dot{\alpha}}^{ \pm}=\mu_{ \pm}^{\alpha} \partial_{\alpha \dot{\alpha}}^{L} \quad \text { with } \partial_{\alpha \dot{\alpha}}^{L}=\frac{\partial}{\partial x_{L}^{\alpha \dot{\alpha}}}
$$

In the real case, when the coordinates $\left(x_{\mathrm{L}}^{\alpha \dot{\alpha}}, \theta^{\alpha i}\right)$ belong to the real chiral superspace $\mathcal{R}_{\mathrm{L}}^{4 \mid 2 \mathcal{N}}$, we have the fibration (6.10), and the vector fields (6.15) and

$$
\bar{V}_{\dot{3}}^{ \pm}=\partial_{\bar{\zeta}_{ \pm}}
$$

can be identified with bosonic vector fields ${ }^{38}$ of type $(0,1)$ on $\mathcal{P}_{*}^{3 \mid \mathcal{N}}$ similar to the vector fields (4.19) and (4.33) as discussed in Section 4 for the self-dual case. As an odd vector field of type $(0,1)$ on $\mathcal{P}_{*}^{3 \mid \mathcal{N}}$, we have

$$
\bar{\partial}_{i}^{+}=\mu_{+}^{\alpha} \frac{\partial}{\partial \theta^{\alpha i}} \quad \text { on } \hat{\mathcal{V}}_{+} \quad \text { and } \quad \bar{\partial}_{i}^{-}=\mu_{-}^{\alpha} \frac{\partial}{\partial \theta^{\alpha i}} \quad \text { on } \hat{\mathcal{V}}_{-}
$$

These bosonic and fermionic vector fields of type $(0,1)$ on $\mathcal{P}_{*}^{3 \mid \mathcal{N}}$ annihilate all complex coordinates (6.1) and (6.2) (or, equivalently, (6.5)) on $\mathcal{P}_{*}^{3 \mid \mathcal{N}}$.

\footnotetext{
${ }^{38}$ In the case $\varepsilon=+1$, this identification only holds for $\tilde{\mathcal{P}}_{*}^{3 \mid \mathcal{N}}=\left.\mathcal{P}_{*}^{3 \mid \mathcal{N}}\right|_{\left|\zeta_{ \pm}\right| \neq 1}$.
} 


\subsection{Anti-self-dual gauge fields}

Consider a holomorphic vector bundle $\mathcal{E}$ over the space $\mathcal{P}_{*}^{3 \mid \mathcal{N}}=\hat{\mathcal{V}}_{+} \cup \hat{\mathcal{V}}_{-}$ defined by a transition function $f_{+-}$on $\hat{\mathcal{V}}_{+} \cap \hat{\mathcal{V}}_{-}$. On $\mathcal{P}_{*}^{3 \mid \mathcal{N}}$, we have

$$
\bar{V}_{\dot{\alpha}}^{+} f_{+-}=0, \quad \partial_{\bar{\zeta}_{+}} f_{+-}=0 \quad \text { and } \quad \bar{\partial}_{i}^{+} f_{+-}=0
$$

since $f_{+-}$is holomorphic. Let us consider trivializations $\psi_{ \pm}$over $\hat{\mathcal{V}}_{ \pm}$similar to (5.6) and (5.7), such that

$$
f_{+-}=\psi_{+}^{-1} \psi_{-} \quad \text { and } \quad \partial_{\bar{\zeta}_{ \pm}} \psi_{ \pm}=0 .
$$

From these equations, we obtain matrix-valued components of a super gauge potential one-form,

$$
\begin{aligned}
\mathcal{A}_{\dot{\alpha}}^{+} & \left.:=\bar{V}_{\dot{\alpha}}^{+}\right\lrcorner \mathcal{A}=\psi_{+} \bar{V}_{\dot{\alpha}}^{+} \psi_{+}^{-1}=\psi_{-} \bar{V}_{\dot{\alpha}}^{+} \psi_{-}^{-1}=\mu_{+}^{\alpha} \mathcal{A}_{\alpha \dot{\alpha}}\left(x_{\mathrm{L}}, \theta\right), \\
\left.\mathcal{A}_{\bar{\zeta}_{+}}:=\partial_{\bar{\zeta}_{+}}\right\lrcorner \mathcal{A} & =\psi_{+} \partial_{\bar{\zeta}_{+}} \psi_{+}^{-1}=\psi_{-} \partial_{\bar{\zeta}_{+}} \psi_{-}^{-1}=0 \\
\mathcal{A}_{i}^{+} & \left.:=\bar{\partial}_{i}^{+}\right\lrcorner \mathcal{A}=\psi_{+} \bar{\partial}_{i}^{+} \psi_{+}^{-1}=\psi_{-} \bar{\partial}_{i}^{+} \psi_{-}^{-1}=\mu_{+}^{\alpha} \mathcal{A}_{\alpha i}\left(x_{\mathrm{L}}, \theta\right),
\end{aligned}
$$

and a linear system

$$
\begin{aligned}
\left(\bar{V}_{\dot{\alpha}}^{+}+\mathcal{A}_{\dot{\alpha}}^{+}\right) \psi_{+} & =0 \\
\partial_{\bar{\zeta}_{+}} \psi_{+} & =0 \\
\left(\bar{\partial}_{i}^{+}+\mathcal{A}_{i}^{+}\right) \psi_{+} & =0
\end{aligned}
$$

where the last equalities in (6.20) and (6.22) follow from the generalized Liouville's theorem on $\mathbb{C} P_{*}^{1}$. The compatibility conditions of the linear system $(6.23)-(6.25)$ read

$$
\begin{aligned}
& {\left[\nabla_{\alpha \dot{\alpha}}, \nabla_{\beta \dot{\beta}}\right]+\left[\nabla_{\beta \dot{\alpha}}, \nabla_{\alpha \dot{\beta}}\right]=0, \quad\left[\nabla_{\alpha i}, \nabla_{\beta \dot{\beta}}\right]+\left[\nabla_{\beta i}, \nabla_{\alpha \dot{\beta}}\right]=0,} \\
& \quad\left\{\nabla_{\alpha i}, \nabla_{\beta j}\right\}+\left\{\nabla_{\beta i}, \nabla_{\alpha j}\right\}=0,
\end{aligned}
$$

where we have - as before - introduced covariant derivatives

$$
\nabla_{\alpha \dot{\alpha}}:=\partial_{\alpha \dot{\alpha}}^{L}+\mathcal{A}_{\alpha \dot{\alpha}} \quad \text { and } \quad \nabla_{\alpha i}:=\partial_{\alpha i}+\mathcal{A}_{\alpha i} .
$$

Equations (6.26) are the anti-self-dual $\mathcal{N}$-extended SYM equations in superspace formulation. ${ }^{39}$

\footnotetext{
${ }^{39}$ Note that fibres $\mathbb{C}_{\zeta}^{2 \mid \mathcal{N}}$ over $\zeta \in \mathbb{C} P_{*}^{1}$ in the fibration (6.4) are the $\alpha_{\mathrm{L}}$-superplanes introduced in Section 5. From (6.23)-(6.26), it follows that $\mathcal{N}$-extended anti-self-duality in superfield formulation is equivalent to flatness of SYM fields on these complex $(2 \mid \mathcal{N})$ dimensional $\alpha_{\mathrm{L}}$-superplanes.
} 
As in the self-dual case, one can rewrite (6.26) in component fields. The full set of equations of motion for $\mathcal{N}=4$ is

$$
\begin{aligned}
& f_{\alpha \beta}:=-\frac{1}{2} \varepsilon^{\dot{\alpha} \dot{\beta}}\left(\partial_{\alpha \dot{\alpha}} A_{\beta \dot{\beta}}-\partial_{\beta \dot{\beta}} A_{\alpha \dot{\alpha}}+\left[A_{\alpha \dot{\alpha}}, A_{\beta \dot{\beta}}\right]\right)=0, \\
& \nabla_{\alpha \dot{\alpha}} \tilde{\chi}_{i}^{\dot{\alpha}}=0, \\
& \square \phi_{i j}+2 \varepsilon\left\{\tilde{\chi}_{i}^{\dot{\alpha}}, \tilde{\chi}_{\dot{\alpha} j}\right\}=0, \\
& \nabla_{\alpha \dot{\alpha}} \chi_{i j k}^{\alpha}-\varepsilon\left[\tilde{\chi}_{\dot{\alpha}[i}, \phi_{j k]}\right]=0, \\
& \varepsilon^{\alpha \gamma} \nabla_{\alpha \dot{\alpha}} G_{\gamma \delta[i j k l]}+12 \varepsilon\left\{\tilde{\chi}_{\dot{\alpha}[i}, \chi_{\delta j k l]}\right\}-18 \varepsilon\left[\phi_{[i j}, \nabla_{\delta \dot{\alpha}} \phi_{k l]}\right]=0,
\end{aligned}
$$

and the cases $\mathcal{N}<4$ are governed by the first $\mathcal{N}+1$ equations.

\subsection{Gauge equivalent trivializations}

One can again consider trivializations similar to (5.21) and (5.22) and transform the linear system (6.23)-(6.25) to a gauge equivalent linear system. Namely, there exist $\tau_{\varepsilon}$-regular matrix-valued functions $\hat{\psi}_{ \pm}$on $\hat{\mathcal{V}}_{ \pm} \subset \mathcal{P}_{*}^{3 \mid \mathcal{N}}$ such that

$$
f_{+-}=\psi_{+}^{-1} \psi_{-}=\hat{\psi}_{+}^{-1} \hat{\psi}_{-} \quad \text { and } \quad \bar{\partial}_{i}^{ \pm} \hat{\psi}_{ \pm}=0 \text { with } \bar{\partial}_{i}^{ \pm}:=\mu_{ \pm}^{\alpha} \partial_{\alpha i},
$$

and therefore the matrix-valued functions

$$
\varphi:=\psi_{+} \hat{\psi}_{+}^{-1}=\psi_{-} \hat{\psi}_{-}^{-1}
$$

generate gauge transformations

$$
\begin{aligned}
\mathcal{A}_{\dot{\alpha}}^{ \pm} & \longmapsto \hat{\mathcal{A}}_{\dot{\alpha}}^{ \pm}=\varphi^{-1} \mathcal{A}_{\dot{\alpha}}^{ \pm} \varphi+\varphi^{-1} \bar{V}_{\dot{\alpha}}^{ \pm} \varphi=\hat{\psi}_{ \pm} \bar{V}_{\dot{\alpha}}^{ \pm} \hat{\psi}_{ \pm}^{-1}, \\
0=\mathcal{A}_{\bar{\zeta}_{ \pm}} \longmapsto \hat{\mathcal{A}}_{\bar{\zeta}_{ \pm}}=\varphi^{-1} \partial_{\bar{\zeta}_{ \pm}} \varphi=\hat{\psi}_{ \pm} \partial_{\bar{\zeta}_{ \pm}} \hat{\psi}_{ \pm}^{-1} & \\
\mathcal{A}_{i}^{ \pm} & \longmapsto \hat{\mathcal{A}}_{i}^{ \pm}=\varphi^{-1} \mathcal{A}_{i}^{ \pm} \varphi+\varphi^{-1} \bar{\partial}_{i}^{ \pm} \varphi=\hat{\psi}_{ \pm} \bar{\partial}_{i}^{ \pm} \hat{\psi}_{ \pm}^{-1}=0 .
\end{aligned}
$$

For the new linear system ${ }^{40}$

$$
\begin{aligned}
\left(\bar{V}_{\dot{\alpha}}^{+}+\hat{\mathcal{A}}_{\dot{\alpha}}^{+}\right) \hat{\psi}_{+} & =0, \\
\left(\partial_{\bar{\zeta}_{+}}+\hat{\mathcal{A}}_{\bar{\zeta}_{+}}\right) \hat{\psi}_{+} & =0, \\
\bar{\partial}_{i}^{+} \hat{\psi}_{+} & =0,
\end{aligned}
$$

the compatibility conditions read

$$
\begin{gathered}
\bar{V}_{\dot{\alpha}}^{+} \hat{\mathcal{A}}_{\dot{\beta}}^{+}-\bar{V}_{\dot{\beta}}^{+} \hat{\mathcal{A}}_{\dot{\alpha}}^{+}+\left[\hat{\mathcal{A}}_{\dot{\alpha}}^{+}, \hat{\mathcal{A}}_{\dot{\beta}}^{+}\right]=0 \\
\partial_{\bar{\zeta}_{+}} \hat{\mathcal{A}}_{\dot{\alpha}}^{+}-\bar{V}_{\dot{\alpha}}^{+} \hat{\mathcal{A}}_{\bar{\zeta}_{+}}+\left[\hat{\mathcal{A}}_{\bar{\zeta}_{+}}, \hat{\mathcal{A}}_{\dot{\alpha}}^{+}\right]=0
\end{gathered}
$$

\footnotetext{
${ }^{40}$ On $\hat{\mathcal{V}}_{-}$, the equations are similar.
} 
The dependence of $\hat{\mathcal{A}}_{\dot{\alpha}}^{+}$and $\hat{\mathcal{A}}_{\bar{\zeta}_{+}}$on $\zeta_{+}$and $\bar{\zeta}_{+}$is fixed by their transformation properties similarly to the self-dual cases (5.33) and (5.34). Namely, for $\mathcal{N}=4$, we have

$$
\begin{aligned}
\hat{\mathcal{A}}_{\dot{\alpha}}^{+}= & \mu_{+}^{\alpha} A_{\alpha \dot{\alpha}}\left(x_{\mathrm{L}}\right)+\theta_{+}^{i} \tilde{\chi}_{\dot{\alpha} i}\left(x_{\mathrm{L}}\right)+\nu_{+} \frac{1}{2 !} \theta_{+}^{i} \theta_{+}^{j} \hat{\mu}_{+}^{\alpha} \phi_{\alpha \dot{\alpha} i j}\left(x_{\mathrm{L}}\right) \\
& +\nu_{+}^{2} \frac{1}{3 !} \theta_{+}^{i} \theta_{+}^{j} \theta_{+}^{k} \hat{\mu}_{+}^{\alpha} \hat{\mu}_{+}^{\beta} \chi_{\alpha \dot{\alpha} \beta i j k}\left(x_{\mathrm{L}}\right) \\
& +\nu_{+}^{3} \frac{1}{4 !} \theta_{+}^{i} \theta_{+}^{j} \theta_{+}^{k} \theta_{+}^{l} \hat{\mu}_{+}^{\alpha} \hat{\mu}_{+}^{\beta} \hat{\mu}_{+}^{\gamma} G_{\alpha \dot{\alpha} \beta \gamma i j k l}\left(x_{\mathrm{L}}\right), \\
\hat{\mathcal{A}}_{\bar{\zeta}_{+}}= & \nu_{+}^{2} \frac{1}{2 !} \theta_{+}^{i} \theta_{+}^{j} \phi_{i j}\left(x_{\mathrm{L}}\right)+\nu_{+}^{3} \frac{1}{3 !} \theta_{+}^{i} \theta_{+}^{j} \theta_{+}^{k} \hat{\mu}_{+}^{\alpha} \chi_{\alpha i j k}\left(x_{\mathrm{L}}\right) \\
& +\nu_{+}^{4} \frac{1}{4 !} \theta_{+}^{i} \theta_{+}^{j} \theta_{+}^{k} \theta_{+}^{l} \hat{\mu}_{+}^{\beta} \hat{\mu}_{+}^{\gamma} G_{\beta \gamma i j k l}\left(x_{\mathrm{L}}\right),
\end{aligned}
$$

where

$\left(\mu_{+}^{\alpha}\right)=\left(\begin{array}{c}-\zeta_{+} \\ 1\end{array}\right), \quad\left(\hat{\mu}_{+}^{\alpha}\right)=\left(\begin{array}{c}\varepsilon \\ -\bar{\zeta}_{+}\end{array}\right)$and $\quad \nu_{+}=\frac{1}{1-\varepsilon \zeta_{+} \bar{\zeta}_{+}}, \zeta_{+} \in V_{+} \subset \mathbb{C} P_{*}^{1}$.

Substituting (6.39) and (6.40) into the field equations (6.37) and (6.38) of hCS theory on the supertwistor space $\mathcal{P}_{*}^{3 \mid 4}$, one obtains the field equations (6.28) for $\mathcal{N}=4$. The appropriate truncation for $\mathcal{N}<4$ is done exactly as in the self-dual case: from the nilpotency of the $\theta_{+}^{i}$ 's it follows that there are less fields in the expansions (6.39) and (6.40), which, in turn, yields the first $\mathcal{N}+1$ equations of $(6.28)$.

Again, we have described a one-to-one correspondence between gauge equivalence classes of solutions to the $\mathcal{N}$-extended anti-SDYM equations on $\left(\mathbb{R}^{4}, g\right)$ and equivalence classes of holomorphic vector bundles $\mathcal{E}$ over the dual supertwistor space $\mathcal{P}_{*}^{3 \mid \mathcal{N}}$, analogously to the self-dual case. The PenroseWard transform here is given by the formulæ (6.39) and (6.40).

\section{A quadric in a product of supertwistor spaces}

\subsection{Coordinates on $\mathcal{P}^{3 \mid 3} \times \mathcal{P}_{*}^{3 \mid 3}$}

Let us fix $\mathcal{N}=3$ and consider the direct product $\mathcal{P}^{3 \mid 3} \times \mathcal{P}_{*}^{3 \mid 3}$ of supertwistor spaces which is an open subset in the supermanifold $\mathbb{C} P^{3 \mid 3} \times \mathbb{C} P_{*}^{3 \mid 3}$. This subset can be covered by four patches $\hat{\mathcal{U}}_{a}, a=1, \ldots, 4$, defined as

$$
\hat{\mathcal{U}}_{1}:=\hat{\mathcal{U}}_{+} \times \hat{\mathcal{V}}_{+}, \quad \hat{\mathcal{U}}_{2}:=\hat{\mathcal{U}}_{-} \times \hat{\mathcal{V}}_{+}, \quad \hat{\mathcal{U}}_{3}:=\hat{\mathcal{U}}_{+} \times \hat{\mathcal{V}}_{-}, \quad \hat{\mathcal{U}}_{4}:=\hat{\mathcal{U}}_{-} \times \hat{\mathcal{V}}_{-},
$$


where $\hat{\mathcal{U}}_{ \pm}$and $\hat{\mathcal{V}}_{ \pm}$are coordinate patches on $\mathcal{P}^{3 \mid 3}$ and $\mathcal{P}_{*}^{3 \mid 3}$, respectively, described in Sections 4 and 6. Thus, we have

$$
\mathcal{P}^{3 \mid 3} \times \mathcal{P}_{*}^{3 \mid 3}=\bigcup_{a=1}^{4} \hat{\mathcal{U}}_{a}
$$

with coordinates

$$
\begin{aligned}
& \left(z_{(1)}^{\alpha}, \lambda_{\dot{\alpha}}^{(1)}, \eta_{i}^{(1)}, w_{(1)}^{\dot{\alpha}}, \mu_{\alpha}^{(1)}, \theta_{(1)}^{i}\right)=\left(z_{+}^{\alpha}, \lambda_{\dot{\alpha}}^{+}, \eta_{i}^{+}, w_{+}^{\dot{\alpha}}, \mu_{\alpha}^{+}, \theta_{+}^{i}\right) \text { on } \hat{\mathcal{U}}_{1} \text {, } \\
& \left(z_{(2)}^{\alpha}, \lambda_{\dot{\alpha}}^{(2)}, \eta_{i}^{(2)}, w_{(2)}^{\dot{\alpha}}, \mu_{\alpha}^{(2)}, \theta_{(2)}^{i}\right)=\left(z_{-}^{\alpha}, \lambda_{\dot{\alpha}}^{-}, \eta_{i}^{-}, w_{+}^{\dot{\alpha}}, \mu_{\alpha}^{+}, \theta_{+}^{i}\right) \text { on } \hat{\mathcal{U}}_{2} \text {, } \\
& \left(z_{(3)}^{\alpha}, \lambda_{\dot{\alpha}}^{(3)}, \eta_{i}^{(3)}, w_{(3)}^{\dot{\alpha}}, \mu_{\alpha}^{(3)}, \theta_{(3)}^{i}\right)=\left(z_{+}^{\alpha}, \lambda_{\dot{\alpha}}^{+}, \eta_{i}^{+}, w_{-}^{\dot{\alpha}}, \mu_{\alpha}^{-}, \theta_{-}^{i}\right) \quad \text { on } \hat{\mathcal{U}}_{3} \text {, } \\
& \left(z_{(4)}^{\alpha}, \lambda_{\dot{\alpha}}^{(4)}, \eta_{i}^{(4)}, w_{(4)}^{\dot{\alpha}}, \mu_{\alpha}^{(4)}, \theta_{(4)}^{i}\right)=\left(z_{-}^{\alpha}, \lambda_{\dot{\alpha}}^{-}, \eta_{i}^{-}, w_{-}^{\dot{\alpha}}, \mu_{\alpha}^{-}, \theta_{-}^{i}\right) \text { on } \hat{\mathcal{U}}_{4} \text {. }
\end{aligned}
$$

On nonempty intersections

$$
\begin{aligned}
& \hat{\mathcal{U}}_{1} \cap \hat{\mathcal{U}}_{2}=\left(\hat{\mathcal{U}}_{+} \cap \hat{\mathcal{U}}_{-}\right) \times \hat{\mathcal{V}}_{+}, \quad \hat{\mathcal{U}}_{1} \cap \hat{\mathcal{U}}_{3}=\hat{\mathcal{U}}_{+} \times\left(\hat{\mathcal{V}}_{+} \cap \hat{\mathcal{V}}_{-}\right), \\
& \hat{\mathcal{U}}_{2} \cap \hat{\mathcal{U}}_{4}=\hat{\mathcal{U}}_{-} \times\left(\hat{\mathcal{V}}_{+} \cap \hat{\mathcal{V}}_{-}\right), \quad \hat{\mathcal{U}}_{3} \cap \hat{\mathcal{U}}_{4}=\left(\hat{\mathcal{U}}_{+} \cap \hat{\mathcal{U}}_{-}\right) \times \hat{\mathcal{V}}_{-}, \\
& \hat{\mathcal{U}}_{1} \cap \hat{\mathcal{U}}_{4}=\left(\hat{\mathcal{U}}_{+} \cap \hat{\mathcal{U}}_{-}\right) \times\left(\hat{\mathcal{V}}_{+} \cap \hat{\mathcal{V}}_{-}\right)=\hat{\mathcal{U}}_{2} \cap \hat{\mathcal{U}}_{3},
\end{aligned}
$$

these coordinates are transformed in an obvious way which follows from the transformations of the coordinates on $\mathcal{P}^{3 \mid 3}$ and $\mathcal{P}_{*}^{3 \mid 3}$. Recall that the coordinates (7.3) are obtained from homogeneous coordinates

$$
\left[\omega^{\alpha}, \lambda_{\dot{\alpha}}, \eta_{i} ; \sigma^{\dot{\alpha}}, \mu_{\alpha}, \theta^{i}\right]
$$

on $\mathbb{C} P^{3 \mid 3} \times \mathbb{C} P_{*}^{3 \mid 3}$.

\subsection{A quadric}

Let us consider a quadric $\mathbb{L}^{5 \mid 6}$ in $\mathbb{C} P^{3 \mid 3} \times \mathbb{C} P_{*}^{3 \mid 3}$ defined by the equation ${ }^{41}$

$$
\omega^{\alpha} \mu_{\alpha}-\sigma^{\dot{\alpha}} \lambda_{\dot{\alpha}}+2 \theta^{i} \eta_{i}=0 \text {. }
$$

Consider also an open subset $\mathcal{L}^{5 \mid 6}$ in $\mathbb{L}^{5 \mid 6}$ as the quadric $\mathbb{L}^{5 \mid 6} \cap\left(\mathcal{P}^{3 \mid 3} \times \mathcal{P}_{*}^{3 \mid 3}\right)$ in $\mathcal{P}^{3 \mid 3} \times \mathcal{P}_{*}^{3 \mid 3}$. The supermanifold $\mathcal{L}^{5 \mid 6}$ is covered by four patches

$$
\mathcal{U}_{a}:=\hat{\mathcal{U}}_{a} \cap \mathbb{L}^{5 \mid 6}, \quad \mathcal{L}^{5 \mid 6}=\bigcup_{a=1}^{4} \mathcal{U}_{a}
$$

\footnotetext{
${ }^{41}$ The space $\mathbb{C} P_{*}^{3 \mid 3}$ is the space of $(3 \mid 3)$-planes in $\mathbb{C}^{4 \mid 3}$. Each such plane is naturally described by a ray, i.e., a (1|0)-dimensional subspaces of $\mathbb{C}^{4 \mid 3}$, orthogonal to the plane. Thus, the space $\mathbb{C} P_{*}^{3 \mid 3}$ is biholomorphic to $\mathbb{C} P^{3 \mid 3}$, which is the space of rays in $\mathbb{C}^{4 \mid 3}$. The quadric is exactly the appropriate orthogonality condition between elements of both projective spaces.
} 
and defined on $\hat{\mathcal{U}}_{a}$ by the equation

$$
z_{(a)}^{\alpha} \mu_{\alpha}^{(a)}-w_{(a)}^{\dot{\alpha}} \lambda_{\dot{\alpha}}^{(a)}+2 \theta_{(a)}^{i} \eta_{i}^{(a)}=0
$$

where no summation over the index $a$ is implied.

Note that one can introduce a holomorphic projection

$$
p: \mathcal{L}^{5 \mid 6} \rightarrow \mathbb{C} P^{1} \times \mathbb{C} P_{*}^{1}
$$

with $(3 \mid 6)$-dimensional fibres. Then $\mathcal{L}^{5 \mid 6}$ can be identified with a quotient bundle whose body $\mathcal{L}^{5}$ is

$$
\mathcal{L}^{5}=\frac{(\mathcal{O}(1,0) \oplus \mathcal{O}(1,0) \oplus \mathcal{O}(0,1) \oplus \mathcal{O}(0,1))}{\mathcal{O}(1,1)}
$$

Here $\mathcal{O}(n, 0)$ is the bundle $\mathcal{O}(n)$ over the projective space $\mathbb{C} P^{1}$ with homogeneous coordinates $\left[\lambda_{\dot{\alpha}}\right], \mathcal{O}(0, n)$ is the bundle $\mathcal{O}(n)$ over the projective space $\mathbb{C} P_{*}^{1}$ with homogeneous coordinates $\left[\mu_{\alpha}\right]$ and $\mathcal{O}(m, n)$ is the line bundle $\mathcal{O}(m, 0) \otimes \mathcal{O}(0, n)$ over $\mathbb{C} P^{1} \times \mathbb{C} P_{*}^{1}$. The quotient by $\mathcal{O}(1,1)$ arises from the quadric condition (7.8) (see e.g., [34]). The base $\mathbb{C} P^{1} \times \mathbb{C} P_{*}^{1}$ of the fibration (7.9) is covered by four patches

$$
V_{a}:=\mathcal{U}_{a} \cap\left(\mathbb{C} P^{1} \times \mathbb{C} P_{*}^{1}\right)
$$

with coordinates $\left(\lambda_{(a)}, \zeta_{(a)}\right)$ on an open set $V_{a} \subset \mathbb{C} P^{1} \times \mathbb{C} P_{*}^{1}$. Recall that $\mathcal{L}^{5 \mid 6}=\bigcup_{a=1}^{4} \mathcal{U}_{a}$ and

$$
\begin{array}{ll}
\left(\lambda_{\dot{\alpha}}^{(1)}\right)^{\top}=\left(1, \lambda_{(1)}\right)=\left(1, \lambda_{+}\right), & \left(\lambda_{\dot{\alpha}}^{(2)}\right)^{\top}=\left(\lambda_{(2)}, 1\right)=\left(\lambda_{-}, 1\right), \\
\left(\lambda_{\dot{\alpha}}^{(3)}\right)^{\top}=\left(1, \lambda_{(3)}\right)=\left(1, \lambda_{+}\right), & \left(\lambda_{\dot{\alpha}}^{(4)}\right)^{\top}=\left(\lambda_{(4)}, 1\right)=\left(\lambda_{-}, 1\right), \\
\left(\mu_{\alpha}^{(1)}\right)^{\top}=\left(1, \zeta_{(1)}\right)=\left(1, \zeta_{+}\right), & \left(\mu_{\alpha}^{(2)}\right)^{\top}=\left(1, \zeta_{(2)}\right)=\left(1, \zeta_{+}\right), \\
\left(\mu_{\alpha}^{(3)}\right)^{\top}=\left(\zeta_{(3)}, 1\right)=\left(\zeta_{-}, 1\right), & \left(\mu_{\alpha}^{(4)}\right)^{\top}=\left(\zeta_{(4)}, 1\right)=\left(\zeta_{-}, 1\right) .
\end{array}
$$

We denote by $z_{(a)}^{A}$ with $A=1,2,3$ bosonic coordinates on the fibres over $V_{a}$ in the bundle (7.9). Additionally, we use odd variables $\theta_{(a)}^{i}$ and $\eta_{i}^{(a)}$ as the fermionic coordinates on these fibres. 


\subsection{Moduli of complex submanifolds}

Holomorphic sections over $V_{a}$ of the bundle (7.9) are spaces $\mathcal{L}_{x, \theta, \eta}^{2 \mid 0} \cong \mathbb{C} P^{1} \times$ $\mathbb{C} P_{*}^{1}$ defined by the equations

$$
\begin{aligned}
& z_{(a)}^{\alpha}=x_{\mathrm{R}}^{\alpha \dot{\alpha}} \lambda_{\dot{\alpha}}^{(a)}, \quad w_{(a)}^{\dot{\alpha}}=x_{\mathrm{L}}^{\alpha \dot{\alpha}} \mu_{\alpha}^{(a)}, \\
& \theta_{(a)}^{i}=\theta^{\alpha i} \mu_{\alpha}^{(a)}, \quad \eta_{i}^{(a)}=\eta_{i}^{\dot{\alpha}} \lambda_{\dot{\alpha}}^{(a)} \text { with }\left(\lambda_{\dot{\alpha}}^{(a)}, \mu_{\alpha}^{(a)}\right) \in V_{a} .
\end{aligned}
$$

These sections are not independent due to equation (7.8), which is solved by the choice

$$
x_{\mathrm{R}}^{\alpha \dot{\alpha}}=x^{\alpha \dot{\alpha}}-\theta^{\alpha i} \eta_{i}^{\dot{\alpha}} \quad \text { and } \quad x_{\mathrm{L}}^{\alpha \dot{\alpha}}=x^{\alpha \dot{\alpha}}+\theta^{\alpha i} \eta_{i}^{\dot{\alpha}} .
$$

We may choose three independent functions from $z_{(a)}^{\alpha}=x_{\mathrm{R}}^{\alpha \dot{\alpha}} \lambda_{\dot{\alpha}}^{(a)}$ and $w_{(a)}^{\dot{\alpha}}=$ $x_{\mathrm{L}}^{\alpha \dot{\alpha}} \mu_{\alpha}^{(a)}$ and raise them to the coordinates $z_{(a)}^{A}$ on the fibres. The local sections (7.13) are properly glued on $V_{a} \cap V_{b} \neq \varnothing$ and therefore define a global holomorphic section of the bundle (7.9) parametrized by supermoduli $(x, \theta, \eta)=\left(x^{\alpha \dot{\alpha}}, \theta^{\alpha i}, \eta_{i}^{\dot{\alpha}}\right) \in \mathbb{C}^{4 \mid 12}$ due to $(7.14)$.

Equations (7.13) with $a=1, \ldots, 4$ define a supertwistor correspondence between $\mathcal{L}^{5 \mid 6}$ and $\mathbb{C}^{4 \mid 12}$ via a double fibration

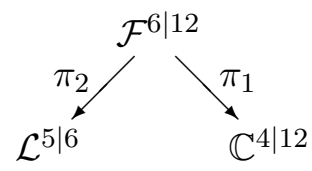

where $\mathcal{F}^{6 \mid 12}:=\mathbb{C}^{4 \mid 12} \times \mathbb{C} P^{1} \times \mathbb{C} P_{*}^{1}$. Namely, a point $(x, \theta, \eta) \in \mathbb{C}^{4 \mid 12}$ corresponds to

$$
\mathcal{L}_{x, \theta, \eta}^{2 \mid 0}=\pi_{2}\left(\pi_{1}^{-1}(x, \theta, \eta)\right) \cong \mathbb{C} P^{1} \times \mathbb{C} P_{*}^{1} \hookrightarrow \mathcal{L}^{5 \mid 6}
$$

and a point $\ell \in \mathcal{L}^{5 \mid 6}$ corresponds to a (1|6)-dimensional null superline (an intersection of the $\alpha$ - and $\beta$-superplanes described in the previous sections) $\pi_{1}\left(\pi_{2}^{-1}(\ell)\right) \hookrightarrow \mathbb{C}^{4 \mid 12}$. Note that for the coordinates (7.3) satisfying the quadric equation (7.8), we have [31]

$$
\begin{aligned}
& \pi_{2}^{*} z_{(a)}^{\alpha}=x_{\mathrm{R}}^{\alpha \dot{\alpha}} \lambda_{\dot{\alpha}}^{(a)}, \quad \pi_{2}^{*} \lambda_{\dot{\alpha}}^{(a)}=\lambda_{\dot{\alpha}}^{(a)}, \quad \pi_{2}^{*} w_{(a)}^{\dot{\alpha}}=x_{\mathrm{L}}^{\alpha \dot{\alpha}} \mu_{\alpha}^{(a)}, \\
& \pi_{2}^{*} \mu_{\alpha}^{(a)}=\mu_{\alpha}^{(a)}, \pi_{2}^{*} \theta_{(a)}^{i}=\theta^{\alpha i} \mu_{\alpha}^{(a)} \quad \text { and } \quad \pi_{2}^{*} \eta_{i}^{(a)}=\eta_{i}^{\dot{\alpha}} \lambda_{\dot{\alpha}}^{(a)},
\end{aligned}
$$

where $\pi_{2}^{*}$ means the pull-back to $\mathcal{F}^{6 \mid 12}$. The supermanifold $\mathcal{F}^{6 \mid 12}$ is covered by four patches

$$
\tilde{\mathcal{U}}_{a}=\mathbb{C}^{4 \mid 12} \times V_{a}
$$


with coordinates

$$
\left(x^{\alpha \dot{\alpha}}, \theta^{\alpha i}, \eta_{i}^{\dot{\alpha}}, \lambda_{\dot{\alpha}}^{(a)}, \mu_{\alpha}^{(a)}\right) \quad \text { equivalent to } \quad\left(x^{\alpha \dot{\alpha}}, \theta^{\alpha i}, \eta_{i}^{\dot{\alpha}}, \lambda_{(a)}, \zeta_{(a)}\right) .
$$

This supermanifold is obviously projected onto $\mathbb{C}^{4 \mid 12}$ with coordinates

$$
\left(x^{\alpha \dot{\alpha}}, \theta^{\alpha i}, \eta_{i}^{\dot{\alpha}}\right)
$$

and onto $\mathcal{L}^{5 \mid 6}$ with coordinates

$$
\begin{gathered}
z_{(a)}^{\alpha}=x_{\mathrm{R}}^{\alpha \dot{\alpha}} \lambda_{\dot{\alpha}}^{(a)}, \quad \lambda_{\dot{\alpha}}^{(a)}, \quad \eta_{i}^{(a)}=\eta_{i}^{\dot{\alpha}} \lambda_{\dot{\alpha}}^{(a)} \text { and } \quad w_{(a)}^{\dot{\alpha}}=x_{\mathrm{L}}^{\alpha \dot{\alpha}} \mu_{\alpha}^{(a)}, \\
\mu_{\alpha}^{(a)}, \theta_{(a)}^{i}=\theta^{\alpha i} \mu_{\alpha}^{(a)}
\end{gathered}
$$

which are not all independent but equivalent to $\left(z_{(a)}^{A}, \lambda_{(a)}, \zeta_{(a)}, \theta_{(a)}^{i}, \eta_{i}^{(a)}\right)$. Note that we are considering the complex superspace $\mathbb{C}^{4 \mid 12}$. Appropriate reality conditions will be discussed later on.

\subsection{Vector fields}

The tangent spaces to the (1|6)-dimensional leaves of the fibration

$$
\pi_{2}: \mathcal{F}^{6 \mid 12} \longrightarrow \mathcal{L}^{5 \mid 6}
$$

from (7.15) are spanned by the holomorphic vector fields

$$
\begin{aligned}
W^{(a)} & :=\mu_{(a)}^{\alpha} \lambda_{(a)}^{\dot{\alpha}} \partial_{\alpha \dot{\alpha}}, \\
D_{(a)}^{i} & =\lambda_{(a)}^{\dot{\alpha}} D_{\dot{\alpha}}^{i} \quad \text { and } \quad D_{i}^{(a)}=\mu_{(a)}^{\alpha} D_{\alpha i},
\end{aligned}
$$

which are properly glued on $\tilde{\mathcal{U}}_{a} \cap \tilde{\mathcal{U}}_{b} \neq \varnothing$ into global vector fields on $\mathcal{F}^{6 \mid 12}$. Here, $D_{\alpha i}$ and $D_{\dot{\alpha}}^{i}$ are vector fields given by (4.18) and (6.13). We shall also consider the antiholomorphic part

$$
\bar{\partial}_{\mathcal{F}}=d \bar{x}^{\alpha \dot{\alpha}} \frac{\partial}{\partial \bar{x}^{\alpha \dot{\alpha}}}+d \bar{\lambda}_{(a)} \frac{\partial}{\partial \bar{\lambda}_{(a)}}+d \bar{\zeta}_{(a)} \frac{\partial}{\partial \bar{\zeta}_{(a)}}+d \bar{\theta}^{i \alpha} \frac{\partial}{\partial \bar{\theta}^{i \alpha}}+d \bar{\eta}_{i}^{\dot{\alpha}} \frac{\partial}{\partial \bar{\eta}_{i}^{\dot{\alpha}}}
$$

of the exterior derivative $d$ on $\tilde{\mathcal{U}}_{a}$.

\section{8 hCS theory on the quadric}

\subsection{Holomorphic vector bundles over $\mathcal{L}^{5 \mid 6}$}

For defining a holomorphic rank $n$ vector bundle $\mathcal{E}$ over $\mathcal{L}^{5 \mid 6}$, one should consider a covering $\left\{\mathcal{U}_{a}\right\}$ of $\mathcal{L}^{5 \mid 6}$ and a collection $\left\{f_{a b}\right\}$ of holomorphic $n \times n$ 
matrices (Čech 1-cocycle) on nonempty intersections $\mathcal{U}_{a} \cap \mathcal{U}_{b}$ such that

$$
f_{a b} f_{b c}=f_{a c}
$$

on $\mathcal{U}_{a} \cap \mathcal{U}_{b} \cap \mathcal{U}_{c} \neq \varnothing$. We restrict ourselves to topologically trivial bundles $\mathcal{E} \rightarrow \mathcal{L}^{5 \mid 6}$, i.e., those for which there exists a collection $\left\{\hat{\psi}_{a}\right\}$ of regular matrix-valued functions (Čech 0-cochain) such that

$$
f_{a b}=\hat{\psi}_{a}^{-1} \hat{\psi}_{b}
$$

on any nonempty intersection $\mathcal{U}_{a} \cap \mathcal{U}_{b}$. Since $f_{a b}$ is holomorphic on a trivialization of $\mathcal{E}$ over $\mathcal{U}_{a}$, we have

$$
\partial_{\bar{z}_{(a)}^{A}} f_{a b}=0, \quad \partial_{\bar{\lambda}_{(a)}} f_{a b}=0=\partial_{\bar{\zeta}_{(a)}} f_{a b}
$$

plus the trivial equations

$$
\frac{\partial}{\partial \bar{\theta}_{(a)}^{i}} f_{a b}=0=\frac{\partial}{\partial \bar{\eta}_{i}^{(a)}} f_{a b} .
$$

Equations (8.3) and (8.4) imply that $f_{a b}$ is a function of the coordinates $\left(z_{(a)}^{A}, \theta_{(a)}^{i}, \eta_{i}^{(a)}\right)$ on $\mathcal{L}^{5 \mid 6}$. Equivalently, the $f_{a b}$ 's are arbitrary functions of the coordinates (7.21) restricted only by the algebraic equations (8.1). However, if one finds a splitting (8.2), then (8.1) is automatically satisfied.

From (8.2) and (8.3), it follows that

$$
\begin{aligned}
& \hat{\psi}_{a} \partial_{\bar{z}_{(a)}^{A}} \hat{\psi}_{a}^{-1}=\hat{\psi}_{b} \partial_{\bar{z}_{(a)}^{A}} \hat{\psi}_{b}^{-1}=: \hat{\mathcal{A}}_{\bar{z}_{(a)}^{A}}, \\
& \hat{\psi}_{a} \partial_{\bar{\lambda}_{(a)}} \hat{\psi}_{a}^{-1}=\hat{\psi}_{b} \partial_{\bar{\lambda}_{(a)}} \hat{\psi}_{b}^{-1}=: \hat{\mathcal{A}}_{\bar{\lambda}_{(a)}}, \\
& \hat{\psi}_{a} \partial_{\bar{\zeta}_{(a)}} \hat{\psi}_{a}^{-1}=\hat{\psi}_{b} \partial_{\bar{\zeta}_{(a)}} \hat{\psi}_{b}^{-1}=: \hat{\mathcal{A}}_{\bar{\zeta}_{(a)}},
\end{aligned}
$$

where $\hat{\mathcal{A}}_{\bar{z}_{(a)}^{A}}, \hat{\mathcal{A}}_{\bar{\lambda}_{(a)}}$ and $\hat{\mathcal{A}}_{\bar{\zeta}_{(a)}}$ are components of a $(0,1)$-form $\hat{\mathcal{A}}^{0,1}$ on $\mathcal{L}^{5 \mid 6}$ restricted to an open set $\mathcal{U}_{a}$ and having zero components along $\partial / \partial \bar{\theta}_{(a)}^{i}$ and $\partial / \partial \bar{\eta}_{i}^{(a)}$ since we assume $\partial \hat{\psi}_{a} / \partial \bar{\theta}_{(a)}^{i}=\partial \hat{\psi}_{a} / \partial \bar{\eta}_{i}^{(a)}=0$, i.e.,

$$
\left.\hat{\mathcal{A}}^{0,1}\right|_{\mathcal{U}_{a}}=\hat{\mathcal{A}}^{(a)}=\hat{\mathcal{A}}_{\bar{z}_{(a)}^{A}} d \bar{z}_{(a)}^{A}+\hat{\mathcal{A}}_{\bar{\lambda}_{(a)}} d \bar{\lambda}_{(a)}+\hat{\mathcal{A}}_{\bar{\zeta}_{(a)}} d \bar{\zeta}_{(a)}
$$

On nonempty intersections $\mathcal{U}_{a} \cap \mathcal{U}_{b}$, we have

$$
\left.\hat{\mathcal{A}}^{0,1}\right|_{\mathcal{U}_{a}}=\left.\hat{\mathcal{A}}^{0,1}\right|_{\mathcal{U}_{b}}
$$

and therefore $(8.5)-(8.7)$ define the $(0,1)$ part of a global gauge potential on the bundle $\mathcal{E}$ over the supermanifold $\mathcal{L}^{5 \mid 6}$. 


\subsection{Super-hCS theory on $\mathcal{L}^{5 \mid 6}$}

Let us introduce the notation

$$
\bar{\partial}_{\mathcal{L}}=d \bar{z}_{(a)}^{A} \partial_{\bar{z}_{(a)}^{A}}+d \bar{\lambda}_{(a)} \partial_{\bar{\lambda}_{(a)}}+d \bar{\zeta}_{(a)} \partial_{\bar{\zeta}_{(a)}}
$$

Then, the definitions (8.5)-(8.9) of the super gauge potential $\hat{\mathcal{A}}^{0,1}=\left\{\hat{\mathcal{A}}^{(a)}\right\}$ can be rewritten as a linear equation

$$
\bar{\partial}_{\mathcal{E}} \hat{\psi}_{a}:=\left(\bar{\partial}_{\mathcal{L}}+\hat{\mathcal{A}}^{(a)}\right) \hat{\psi}_{a}=0
$$

on unknown regular matrix-valued functions ${ }^{42} \hat{\psi}_{a}$ for given $\hat{\mathcal{A}}^{(a)}=\left.\hat{\mathcal{A}}^{0,1}\right|_{\mathcal{U}_{a}}$, $a=1, \ldots, 4$. This linear equation reads in components as

$$
\begin{gathered}
\left(\partial_{\bar{z}_{(a)}^{A}}+\hat{\mathcal{A}}_{\bar{z}_{(a)}^{A}}\right) \hat{\psi}_{a}=0, \\
\left(\partial_{\bar{\lambda}_{(a)}}+\hat{\mathcal{A}}_{\bar{\lambda}_{(a)}}\right) \hat{\psi}_{a}=0, \\
\left(\partial_{\bar{\zeta}_{(a)}}+\hat{\mathcal{A}}_{\bar{\zeta}_{(a)}}\right) \hat{\psi}_{a}=0 .
\end{gathered}
$$

The compatibility condition of this linear system is the equation

$$
\bar{\partial}_{\mathcal{L}} \hat{\mathcal{A}}^{(a)}+\hat{\mathcal{A}}^{(a)} \wedge \hat{\mathcal{A}}^{(a)}=0
$$

which is simply the field equation $\left.{ }^{43} \mathcal{F}^{0,2}\right|_{\mathcal{U}_{a}}=0$ of hCS theory on the supermanifold $\mathcal{L}^{5 \mid 6}$.

\subsection{A special gauge}

Note that restrictions of a vector bundle $\mathcal{E} \rightarrow \mathcal{L}^{5 \mid 6}$ to the fibres $\mathbb{C}_{\lambda, \zeta}^{3 \mid 6}$ of the bundle (7.9) are holomorphically trivial since all these fibres are contractible.

\footnotetext{
${ }^{42}$ Recall that $\left(\partial / \partial \bar{\theta}_{(a)}^{i}\right) \hat{\psi}_{a}=\left(\partial / \partial \bar{\eta}_{i}^{(a)}\right) \hat{\psi}_{a}=0$.

${ }^{43}$ Note that solutions to these equations can be constructed via a generalization of the Riemann-Hilbert problem based on the equivalence of the Čech and the Dolbeault description of holomorphic bundles $[42,43]$.
} 
Therefore, there exist trivializations $\tilde{\psi}_{a}$ of $\mathcal{E}$ over $\mathcal{U}_{a}$ such that

$$
f_{a b}=\hat{\psi}_{a}^{-1} \hat{\psi}_{b}=\tilde{\psi}_{a}^{-1} \tilde{\psi}_{b} \text { on } \mathcal{U}_{a} \cap \mathcal{U}_{b} \neq \varnothing
$$

and

$$
\partial_{\bar{z}_{(a)}^{A}} \tilde{\psi}_{a}=0 \text { on } \mathcal{U}_{a}
$$

From (8.16) and (8.17), it follows that the globally defined regular matrixvalued function

$$
\tilde{\varphi}=\hat{\psi}_{a} \tilde{\psi}_{a}^{-1}=\hat{\psi}_{b} \tilde{\psi}_{b}^{-1}
$$

on $\mathcal{L}^{5 \mid 6}$ is an element of the group of gauge transformations, whose action yields

$$
\begin{aligned}
\hat{\mathcal{A}}_{\bar{z}_{(a)}^{A}} \longmapsto \tilde{\mathcal{A}}_{\bar{z}_{(a)}^{A}} & =\tilde{\varphi} \hat{\mathcal{A}}_{\bar{z}_{(a)}^{A}} \tilde{\varphi}^{-1}+\tilde{\varphi} \partial_{\bar{z}_{(a)}^{A}} \tilde{\varphi}^{-1}=\tilde{\psi}_{a} \partial_{\bar{z}_{(a)}^{A}} \tilde{\psi}_{a}^{-1}=0, \\
\hat{\mathcal{A}}_{\bar{\lambda}_{(a)}} \longmapsto \tilde{\mathcal{A}}_{\bar{\lambda}_{(a)}} & =\tilde{\varphi} \hat{\mathcal{A}}_{\bar{\lambda}_{(a)}} \tilde{\varphi}^{-1}+\tilde{\varphi} \partial_{\bar{\lambda}_{(a)}} \tilde{\varphi}^{-1}=\tilde{\psi}_{a} \partial_{\bar{\lambda}_{(a)}} \tilde{\psi}_{a}^{-1}, \\
\hat{\mathcal{A}}_{\bar{\zeta}_{(a)}} \longmapsto \tilde{\mathcal{A}}_{\bar{\zeta}_{(a)}} & =\tilde{\varphi} \hat{\mathcal{A}}_{\bar{\zeta}_{(a)}} \tilde{\varphi}^{-1}+\tilde{\varphi} \partial_{\bar{\zeta}_{(a)}} \tilde{\varphi}^{-1}=\tilde{\psi}_{a} \partial_{\bar{\zeta}_{(a)}} \tilde{\psi}_{a}^{-1} .
\end{aligned}
$$

The linear system (8.12)-(8.14) is correspondingly transformed to

$$
\begin{array}{r}
\partial_{\bar{z}_{(a)}^{A}} \tilde{\psi}_{a}=0, \\
\left(\partial_{\bar{\lambda}_{(a)}}+\tilde{\mathcal{A}}_{\bar{\lambda}_{(a)}}\right) \tilde{\psi}_{a}=0, \\
\left(\partial_{\bar{\zeta}_{(a)}}+\tilde{\mathcal{A}}_{\bar{\zeta}_{(a)}}\right) \tilde{\psi}_{a}=0,
\end{array}
$$

which in coordinate independent form reads

$$
\left(\bar{\partial}_{\mathcal{L}}+\tilde{\mathcal{A}}^{(a)}\right) \tilde{\psi}_{a}=0
$$

In this new gauge described by equations (8.16)-(8.18), the field equations

$$
\bar{\partial}_{\mathcal{L}} \tilde{\mathcal{A}}^{(a)}+\tilde{\mathcal{A}}^{(a)} \wedge \tilde{\mathcal{A}}^{(a)}=0
$$

of super-hCS theory on $\mathcal{L}^{5 \mid 6}$ are simplified to the equations

$$
\partial_{\bar{z}_{(a)}^{A}} \tilde{\mathcal{A}}_{\bar{\lambda}_{(a)}}=0=\partial_{\bar{z}_{(a)}^{A}} \tilde{\mathcal{A}}_{\bar{\zeta}_{(a)}}
$$

and the equation

$$
\partial_{\bar{\lambda}_{(a)}} \tilde{\mathcal{A}}_{\bar{\zeta}_{(a)}}-\partial_{\bar{\zeta}_{(a)}} \tilde{\mathcal{A}}_{\bar{\lambda}_{(a)}}+\left[\tilde{\mathcal{A}}_{\bar{\lambda}_{(a)}}, \tilde{\mathcal{A}}_{\bar{\zeta}_{(a)}}\right]=0
$$

on an open set $V_{a}=\mathcal{U}_{a} \cap\left(\mathbb{C} P^{1} \times \mathbb{C} P_{*}^{1}\right) \subset \mathcal{L}^{5 \mid 6}$ with gluing conditions

$$
\tilde{\mathcal{A}}_{\bar{\lambda}_{(a)}} d \bar{\lambda}_{(a)}+\tilde{\mathcal{A}}_{\bar{\zeta}_{(a)}} d \bar{\zeta}_{(a)}=\tilde{\mathcal{A}}_{\bar{\lambda}_{(b)}} d \bar{\lambda}_{(b)}+\tilde{\mathcal{A}}_{\bar{\zeta}_{(b)}} d \bar{\zeta}_{(b)}
$$

on nonempty intersections $V_{a} \cap V_{b}$ with $a, b=1, \ldots, 4$. Thus, three of five components of the gauge potential $\tilde{\mathcal{A}}^{0,1}$ on $\mathcal{L}^{5 \mid 6}$ satisfying equation (8.15) are gauge equivalent to zero. However, even after transforming to the gauge (8.19)-(8.21), the new gauge potential $\tilde{\mathcal{A}}^{0,1}$ contains information on the extra 
dimensions by depending holomorphically on the coordinates $z_{(a)}^{A}, \theta_{(a)}^{i}$ and $\eta_{i}^{(a)}$. Note that one can also choose $\left\{\tilde{\psi}_{a}\right\}$ such that a linear combination of components $\tilde{\mathcal{A}}_{\bar{z}_{(a)}^{A}}$, say a component

$$
\left.\tilde{\mathcal{A}}_{\bar{\rho}_{(a)}}=\bar{X}^{(a)}\right\lrcorner \tilde{\mathcal{A}}^{0,1}=\bar{X}_{(a)}^{A} \tilde{\mathcal{A}}_{\bar{z}_{(a)}^{A}}
$$

along a vector field $\bar{X}^{(a)}=\bar{X}_{(a)}^{A} \partial_{\bar{z}_{(a)}^{A}}=\partial_{\bar{\rho}_{(a)}}$, will be nonzero. In such a gauge, one will have three nonzero components $\tilde{\mathcal{A}}_{\bar{\lambda}_{(a)}}, \tilde{\mathcal{A}}_{\bar{\zeta}_{(a)}}$ and $\tilde{\mathcal{A}}_{\bar{\rho}_{(a)}}$, which may in principle be used for constructing an action of type (1.2) on the supermanifold $\mathcal{L}^{5 \mid 6}$.

\section{Supertwistors and the full $\mathcal{N}=4 \mathrm{SYM}$ theory}

In this section we shall consider $\mathcal{N}=3 \mathrm{SYM}$ theory which is known to be equivalent to the $\mathcal{N}=4$ SYM theory when formulated on $\mathbb{R}^{4}$. More explicitly, we shall consider the integrability of SYM fields on null superlines, which turns out to be equivalent to the equations of motion of $\mathcal{N}=3$ SYM theory $[26,30-33]$, and its relation with super-hCS theory on a (5|6)dimensional supermanifold.

\subsection{Pulled-back bundle}

Let us consider a holomorphic vector bundle $\mathcal{E} \rightarrow \mathcal{L}^{5 \mid 6}$ and the pulled-back bundle $\pi_{2}^{*} \mathcal{E}$ over the supermanifold $\mathcal{F}^{6 \mid 12}$ with a covering $\left\{\tilde{\mathcal{U}}_{a}\right\}$ given by (7.18) and (7.19). Pull-backs ${ }^{44}$ of the transition functions $\left\{f_{a b}\right\}$ of $\mathcal{E}$ to $\pi_{2}^{*} \mathcal{E}$ are constant along the fibres of $\pi_{2}$, i.e.,

$$
W^{(a)} f_{a b}=D_{(a)}^{i} f_{a b}=D_{i}^{(a)} f_{a b}=0
$$

and due to $\pi_{2}^{*} \bar{\partial}_{\mathcal{L}}=\bar{\partial}_{\mathcal{F}} \circ \pi_{2}^{*}$, they also satisfy $\pi_{2}^{*} \bar{\partial}_{\mathcal{L}} f_{a b}:=\bar{\partial}_{\mathcal{F}} f_{a b}=0$, where $\bar{\partial}_{\mathcal{F}}$ is the antiholomorphic part $(7.25)$ of the exterior derivative on $\mathcal{F}^{6 / 12}$. On the pulled-back trivializations $\left\{\tilde{\psi}_{a}\right\}$ from (8.16) and (8.17), one can also

\footnotetext{
${ }^{44}$ For simplicity, we denote the pulled-back transition functions also by $f_{a b}$, slightly abusing notation. The same holds true for functions $\hat{\psi}_{a}$ and $\tilde{\psi}_{a}$.
} 
impose the conditions

$$
W^{(a)} \tilde{\psi}_{a}=0, \quad D_{(a)}^{i} \tilde{\psi}_{a}=0 \quad \text { and } \quad D_{i}^{(a)} \tilde{\psi}_{a}=0
$$

since the fibres of $\pi_{2}$ are contractible. ${ }^{45}$ So, for $\pi_{2}^{*} \mathcal{E}$, we have transition functions $\left\{f_{a b}\right\}$ split as $f_{a b}=\tilde{\psi}_{a}^{-1} \tilde{\psi}_{b}$ on $\tilde{\mathcal{U}}_{a} \cap \tilde{\mathcal{U}}_{b} \subset \mathcal{F}^{6 \mid 12}$ and satisfying equations (9.1). Moreover, regular matrix-valued functions $\tilde{\psi}_{a}$ 's defining a trivialization of $\pi_{2}^{*} \mathcal{E}$ over $\tilde{\mathcal{U}}_{a}$ satisfy $(9.2)$ with $a=1, \ldots, 4$.

\subsection{Holomorphic triviality on subspaces}

Let us now consider holomorphic vector bundles $\mathcal{E}$ over $\mathcal{L}^{5 \mid 6}$ such that their restriction to any submanifold $\mathcal{L}_{x, \theta, \eta}^{2 \mid 0} \cong \mathbb{C} P^{1} \times \mathbb{C} P_{*}^{1}$ in $\mathcal{L}^{5 \mid 6}$ is holomorphically trivial or, equivalently, such that $\pi_{2}^{*} \mathcal{E}$ is trivial along the fibres of $\pi_{1}$. For such bundles, there exist trivializations $\left\{\psi_{a}\right\}$ of $\pi_{2}^{*} \mathcal{E}$ over $\tilde{\mathcal{U}}_{a}$ such that

$$
f_{a b}=\tilde{\psi}_{a}^{-1} \tilde{\psi}_{b}=\psi_{a}^{-1} \psi_{b} \text { on } \mathcal{U}_{a} \cap \mathcal{U}_{b} \neq \varnothing
$$

and

$$
\bar{\partial}_{\mathcal{F}} \psi_{a}=0
$$

i.e., the regular matrix-valued functions $\psi_{a}$ are holomorphic in the coordinates on $\mathcal{F}^{6 \mid 12}$. It follows from $(9.3)$ that

$$
\phi:=\psi_{a} \tilde{\psi}_{a}^{-1}=\psi_{b} \tilde{\psi}_{b}^{-1}
$$

is a globally defined regular matrix-valued (super)function on $\mathcal{F}^{6 \mid 12}$ which generates gauge transformations

$$
\tilde{\psi}_{a} \longmapsto \psi_{a}=\phi \tilde{\psi}_{a} \text { for } a=1, \ldots, 4
$$

such that

$$
\tilde{\mathcal{A}}^{(a)} \longmapsto \mathcal{A}^{(a)}=\phi \tilde{\mathcal{A}}^{(a)} \phi^{-1}+\phi \bar{\partial}_{\mathcal{F}} \phi^{-1}=0
$$

and

$$
\begin{aligned}
0 & =\tilde{\psi}_{a} D_{(a)}^{i} \tilde{\psi}_{a}^{-1}=: \tilde{\mathcal{A}}_{(a)}^{i} \longmapsto \mathcal{A}_{(a)}^{i}=\phi D_{(a)}^{i} \phi^{-1}=\psi_{a} D_{(a)}^{i} \psi_{a}^{-1}=\psi_{b} D_{(a)}^{i} \psi_{b}^{-1} \\
& =\lambda_{(a)}^{\dot{\alpha}} A_{\dot{\alpha}}^{i}(x, \theta, \eta) \\
0 & =\tilde{\psi}_{a} D_{i}^{(a)} \tilde{\psi}_{a}^{-1}=: \tilde{\mathcal{A}}_{i}^{(a)} \longmapsto \mathcal{A}_{i}^{(a)}=\phi D_{i}^{(a)} \phi^{-1}=\psi_{a} D_{i}^{(a)} \psi_{a}^{-1} \\
& =\psi_{b} D_{i}^{(a)} \psi_{b}^{-1}=\mu_{(a)}^{\alpha} A_{\alpha i}(x, \theta, \eta) \\
0 & =\tilde{\psi}_{a} W^{(a)} \tilde{\psi}_{a}^{-1}=: \tilde{\mathcal{A}}_{w_{(a)}} \longmapsto \mathcal{A}_{w_{(a)}}=\phi W^{(a)} \phi^{-1}=\psi_{a} W^{(a)} \psi_{a}^{-1} \\
& =\psi_{b} W^{(a)} \psi_{b}^{-1}=\mu_{(a)}^{\alpha} \lambda_{(a)}^{\dot{\alpha}} A_{\alpha \dot{\alpha}}(x, \theta, \eta)
\end{aligned}
$$

\footnotetext{
${ }^{45}$ The same conditions $(9.2)$ can be imposed on the pulled-back trivializations $\left\{\hat{\psi}_{a}\right\}$ from (8.2).
} 
where $(x, \theta, \eta)=\left(x^{\alpha \dot{\alpha}}, \theta^{\alpha i}, \eta_{i}^{\dot{\alpha}}\right)$. Note that the last equalities in (9.8)-(9.10) follow from a generalized Liouville theorem on $\mathbb{C} P^{1} \times \mathbb{C} P_{*}^{1}$ which says that $\mathcal{A}_{(a)}^{i}$ is a local section of the bundle $\mathcal{O}(1,0), \mathcal{A}_{i}^{(a)}$ is a local section of the bundle $\mathcal{O}(0,1)$ and $\mathcal{A}_{w_{(a)}}$ is a local section of the bundle $\mathcal{O}(1,1)$ over $\mathbb{C} P^{1} \times$ $\mathbb{C} P_{*}^{1}$.

\subsection{Linear system}

Equations (9.8)-(9.10) can be rewritten as the linear system

$$
\begin{aligned}
\mu_{(a)}^{\alpha} \lambda_{(a)}^{\dot{\alpha}}\left(\partial_{\alpha \dot{\alpha}}+A_{\alpha \dot{\alpha}}\right) \psi_{a} & =0 \\
\lambda_{(a)}^{\dot{\alpha}}\left(D_{\dot{\alpha}}^{i}+A_{\dot{\alpha}}^{i}\right) \psi_{a} & =0 \\
\mu_{(a)}^{\alpha}\left(D_{\alpha i}+A_{\alpha i}\right) \psi_{a} & =0 .
\end{aligned}
$$

By construction, the new linear system (9.11)-(9.13) together with (9.4) is gauge equivalent to the linear system (8.22)-(8.24) together with (9.2).

\subsection{Full $\mathcal{N}=3$ SYM equations}

The linear system (9.11)-(9.13) has been known for a long time $[26,30,32$, 33]. Its compatibility conditions read

$$
\begin{gathered}
\left\{\nabla_{\dot{\alpha}}^{i}, \nabla_{\dot{\beta}}^{j}\right\}+\left\{\nabla_{\dot{\beta}}^{i}, \nabla_{\dot{\alpha}}^{j}\right\}=0, \quad\left\{\nabla_{\alpha i}, \nabla_{\beta j}\right\}+\left\{\nabla_{\beta i}, \nabla_{\alpha j}\right\}=0, \\
\left\{\nabla_{\alpha i}, \nabla_{\dot{\alpha}}^{j}\right\}-2 \delta_{i}^{j} \nabla_{\alpha \dot{\alpha}}=0,
\end{gathered}
$$

where

$$
\nabla_{\alpha \dot{\alpha}}=\partial_{\alpha \dot{\alpha}}+A_{\alpha \dot{\alpha}}, \quad \nabla_{\alpha i}=D_{\alpha i}+A_{\alpha i} \quad \text { and } \quad \nabla_{\dot{\alpha}}^{i}=D_{\dot{\alpha}}^{i}+A_{\dot{\alpha}}^{i}
$$

are gauge covariant derivatives in the superspace $\mathbb{C}^{4 \mid 12}$. Equations (9.14) for the components $\left(A_{\alpha \dot{\alpha}}(x, \theta, \eta), A_{\alpha i}(x, \theta, \eta), A_{\dot{\alpha}}^{i}(x, \theta, \eta)\right)$ of a superconnection are equivalent to the equations of motion of the full $\mathcal{N}=3$ SYM theory $[26,31,33]$. Using the expansions of the superfields (i.e., of the components of the superconnection) in the odd variables, one can rewrite (9.14) as equations on a supermultiplet of ordinary fields. Moreover, these equations turn out to be equivalent to the equations of motion of $\mathcal{N}=4 \mathrm{SYM}$ theory in ordinary space. 
Summarizing, we have (implicitly) described the Penrose-Ward transform

$$
\mathcal{P W}:\left(\hat{\mathcal{A}}^{0,1}\right) \longmapsto\left(f_{\alpha \beta}, \chi_{\alpha i}, \phi_{i j}, \tilde{\chi}_{\dot{\alpha}}^{i}, f_{\dot{\alpha} \dot{\beta}}\right)
$$

which maps solutions of hCS theory on $\mathcal{L}^{5 \mid 6}$ to solutions of $\mathcal{N}=4 \mathrm{SYM}$ theory on $\mathbb{C}^{4}$. Note that the existence of a gauge in which $\mathcal{A}_{\bar{\lambda}_{ \pm}}=0=\mathcal{A}_{\bar{\zeta}_{ \pm}}$ is equivalent to holomorphic triviality of the bundle $\mathcal{E} \rightarrow \mathcal{L}^{5 \mid 6}$ on $\mathcal{L}_{x, \theta, \eta}^{2 \mid 0} \cong$ $\mathbb{C} P^{1} \times \mathbb{C} P_{*}^{1} \hookrightarrow \mathcal{L}^{5 \mid 6}$. Note furthermore that the moduli space of such bundles is a subset of the moduli space of all topologically trivial holomorphic bundles $\mathcal{E}$ over $\mathcal{L}^{5 \mid 6}[34,45]$. This means that the solution space of hCS theory on $\mathcal{L}^{5 \mid 6}$ is larger than that of $\mathcal{N}=4$ SYM theory.

\subsection{Looking for an action}

We saw that the full set of equations of motion for $\mathcal{N}=3 \mathrm{SYM}$ theory is encoded in the equation $\mathcal{F}^{0,2}=0$ on the supermanifold $\mathcal{L}^{5 \mid 6}$ which is the quadric in an open subset of $\mathbb{C} P^{3 \mid 3} \times \mathbb{C} P_{*}^{3 \mid 3}$. One might wonder whether there is some action principle for super-hCS theory on this space. For complex three-dimensional supermanifolds, this is the hCS action (1.2). In the case of the (5|6)-dimensional supermanifold $\mathcal{L}^{5 \mid 6}$, the situation is less clear. Recall that this space is a CY supermanifold and thus it comes with a holomorphic volume form $\Omega^{5 \mid 6}$. Therefore, a possible ansatz is

$$
S=\int \Omega^{5 \mid 6} \wedge \operatorname{tr}\left(\hat{\mathcal{A}}^{0,1} \wedge \bar{\partial} \hat{\mathcal{A}}^{0,1}+\frac{2}{3} \hat{\mathcal{A}}^{0,1} \wedge \hat{\mathcal{A}}^{0,1} \wedge \hat{\mathcal{A}}^{0,1}\right) \wedge \omega^{0,2}
$$

where we abbreviated $\hat{\mathcal{A}}^{0,1}=\hat{\mathcal{A}}^{0,1 \mid 0,0}$ and $\omega^{0,2}=\omega^{0,2 \mid 0,0}$. For this ansatz to be correct, $\omega^{0,2}$ must be nowhere vanishing (otherwise the total measure would be degenerate). Furthermore, the partial integration used for deriving the equations of motion demands that $\omega^{0,2}$ is partially closed, i.e., it has to satisfy the equation $\hat{\mathcal{A}}^{0,1} \wedge \bar{\partial} \omega^{0,2}=0$. It is not clear whether such a $(0,2)$ form exists on $\mathcal{L}^{5 \mid 6}$. Even less clear is the relation of the action (9.17) with string field theory (SFT) for the target space $\mathcal{L}^{5 \mid 6}$. Therefore, we leave this discussion to forthcoming work. ${ }^{46}$

\section{Reality conditions on the quadric}

In the purely bosonic case, one can introduce real (antihermitean) gauge fields on $\mathbb{R}^{4}$ with a metric $g$ of Euclidean signature $(4,0)$, Kleinian signature

\footnotetext{
${ }^{46}$ Another possibility to obtain equation (8.15) is to use an action of holomorphic BF-type theories [42]. However, the relation of this kind of action with SFT is also unclear.
} 
$(2,2)$ or Minkowski signature $(3,1)$ by choosing an appropriate real structure on $\mathbb{C}^{4}$. However, as already mentioned in Section 4 , on the superspace $\mathbb{C}^{4 \mid 4 \mathcal{N}}$, there exists a real structure defining a Euclidean superspace only for an even number of supersymmetries. For simplicity, we restrict ourselves here to the Kleinian and Minkowskian cases.

\subsection{Real structure $\tau_{1}$}

The Kleinian signature $(2,2)$ is related to antilinear transformations ${ }^{47} \tau_{1}$ of spinors defined in Sections 2 and 4. Recall that

$$
\begin{aligned}
& \tau_{1}\left(\begin{array}{l}
\omega^{1} \\
\omega^{2}
\end{array}\right)=\left(\begin{array}{l}
\bar{\omega}^{2} \\
\bar{\omega}^{1}
\end{array}\right), \quad \tau_{1}\left(\begin{array}{l}
\lambda_{\dot{i}} \\
\lambda_{\dot{2}}
\end{array}\right)=\left(\begin{array}{l}
\bar{\lambda}_{\dot{2}} \\
\bar{\lambda}_{\dot{1}}
\end{array}\right), \\
& \tau_{1}\left(\begin{array}{l}
\sigma^{\dot{1}} \\
\sigma^{\dot{2}}
\end{array}\right)=\left(\begin{array}{l}
\bar{\sigma}^{\dot{2}} \\
\bar{\sigma}^{\dot{1}}
\end{array}\right), \quad \tau_{1}\left(\begin{array}{l}
\mu_{1} \\
\mu_{2}
\end{array}\right)=\left(\begin{array}{l}
\bar{\mu}_{2} \\
\bar{\mu}_{1}
\end{array}\right),
\end{aligned}
$$

and obviously $\tau_{1}^{2}=1$. Correspondingly, for $\left(\lambda_{ \pm}, \zeta_{ \pm}\right) \in \mathbb{C} P^{1} \times \mathbb{C} P_{*}^{1}$, we have

$$
\tau_{1}\left(\lambda_{+}\right)=\frac{1}{\bar{\lambda}_{+}}=\bar{\lambda}_{-}, \quad \tau_{1}\left(\zeta_{+}\right)=\frac{1}{\bar{\zeta}_{+}}=\bar{\zeta}_{-}
$$

with stable points

$$
\left\{(\lambda, \zeta) \in \mathbb{C} P^{1} \times \mathbb{C} P_{*}^{1}: \lambda \bar{\lambda}=1, \zeta \bar{\zeta}=1\right\}=S^{1} \times S_{*}^{1} \subset \mathbb{C} P^{1} \times \mathbb{C} P_{*}^{1}
$$

parametrizing a torus $S^{1} \times S_{*}^{1}$. For the coordinates $\left(x^{\alpha \dot{\alpha}}\right)$, we have

$$
\tau_{1}\left(\begin{array}{ll}
x^{1 \dot{1}} & x^{1 \dot{2}} \\
x^{2 \dot{1}} & x^{2 \dot{2}}
\end{array}\right)=\left(\begin{array}{ll}
0 & 1 \\
1 & 0
\end{array}\right)\left(\begin{array}{ll}
\bar{x}^{1 \dot{1}} & \bar{x}^{1 \dot{2}} \\
\bar{x}^{2 \dot{1}} & \bar{x}^{2 \dot{2}}
\end{array}\right)\left(\begin{array}{ll}
0 & 1 \\
1 & 0
\end{array}\right)=\left(\begin{array}{ll}
\bar{x}^{2 \dot{2}} & \bar{x}^{2 \dot{1}} \\
\bar{x}^{1 \dot{2}} & \bar{x}^{1 \dot{1}}
\end{array}\right)
$$

and the real subspace $\mathbb{R}^{4}$ of $\mathbb{C}^{4}$ invariant under the involution $\tau_{1}$ is defined by the equations

$$
x^{2 \dot{2}}=\bar{x}^{1 \dot{1}}=:-\left(x^{4}+\mathrm{i} x^{3}\right) \text { and } x^{2 \dot{1}}=\bar{x}^{1 \dot{2}}=:-\left(x^{2}-\mathrm{i} x^{1}\right)
$$

with a metric $d s^{2}=\operatorname{det}\left(d x^{\alpha \dot{\alpha}}\right)$ of signature $(2,2)$. Recall also that

$$
\tau_{1}\left(\begin{array}{c}
\theta^{1 i} \\
\theta^{2 i}
\end{array}\right)=\left(\begin{array}{c}
\bar{\theta}^{2 i} \\
\bar{\theta}^{1 i}
\end{array}\right), \quad \tau_{1}\left(\begin{array}{c}
\eta_{i}^{\mathrm{i}} \\
\eta_{i}^{\dot{2}}
\end{array}\right)=\left(\begin{array}{c}
\bar{\eta}_{i}^{\dot{2}} \\
\bar{\eta}_{i}^{\mathrm{i}}
\end{array}\right)
$$

and therefore real (Majorana) fermions satisfy (cf. (4.29))

$$
\tau_{1}(\theta)=\theta \Longleftrightarrow \theta^{2 i}=\bar{\theta}^{1 i} \quad \text { and } \quad \tau_{1}(\eta)=\eta \Longleftrightarrow \eta_{i}^{\dot{2}}=\bar{\eta}_{i}^{\dot{1}} .
$$

\footnotetext{
${ }^{47}$ We will not consider the map $\tau_{0}$ here.
} 


\subsection{A $\tau_{1}$-real twistor diagram}

Imposing conditions (10.5) and (10.7) for $\mathcal{N}=3$, we obtain the real superspace $\mathbb{R}^{4 \mid 12}$ as a fixed point set of the involution $\tau_{1}: \mathbb{C}^{4 \mid 12} \rightarrow \mathbb{C}^{4 \mid 12}$. Analogously, for the supertwistor space $\mathbb{C} P^{3 \mid 3}$ and its open subset $\mathcal{P}^{3 \mid 3}$, we obtain real subspaces $\mathbb{R} P^{3 \mid 3}$ and $\mathcal{T}^{3 \mid 3}$ (cf. (2.14)). Accordingly, a real form of the superspace $\mathcal{F}^{6 \mid 12}$ is

$$
\mathcal{F}_{\tau_{1}}^{6 \mid 12}:=\mathbb{R}^{4 \mid 12} \times S^{1} \times S_{*}^{1},
$$

and we have a real quadric

$$
\mathcal{L}_{\tau_{1}}^{5 \mid 6} \subset \mathcal{T}^{3 \mid 3} \times \mathcal{T}_{*}^{3 \mid 3}
$$

as the subset of fixed points of the involution ${ }^{48} \tau_{1}: \mathcal{L}^{5 \mid 6} \rightarrow \mathcal{L}^{5 \mid 6}$. This quadric is defined by equations (7.8)-(7.14) with $x^{\alpha \dot{\alpha}}, \theta^{\alpha i}$ and $\eta_{i}^{\dot{\alpha}}$ satisfying (10.5) and (10.7) with $\lambda_{+}=\mathrm{e}^{\mathrm{i} \chi_{1}}=\lambda_{-}^{-1}, \zeta_{+}=\mathrm{e}^{\mathrm{i} \chi_{2}}=\zeta_{-}^{-1}, 0 \leq \chi_{1}, \chi_{2}<2 \pi$. Thus, we obtain a real form

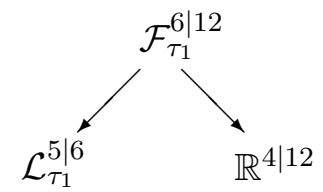

of the double fibration (7.15). One can restrict all (super)functions defined on spaces in (7.15) to the real subspaces in (10.10).

\subsection{Reality of fields in the Kleinian case}

For imposing reality conditions on the functions $\psi_{a}$ (and $f_{a b}$ ) inducing antihermiticity of the fields of $\mathcal{N}=3$ (and $\mathcal{N}=4$ ) SYM theory via the twistor correspondence, it is convenient to consider an open neighbourhood (and an analytic continuation of the functions to a complex domain) of all these real spaces. In fact, for our purpose it is enough to consider the supermanifold

$$
\begin{aligned}
\tilde{\mathcal{F}}^{8 \mid 12} & =\mathbb{R}^{4 \mid 12} \times\left(U_{+} \cap U_{-}\right) \times\left(V_{+} \cap V_{-}\right)=\mathbb{R}^{4 \mid 12} \times\left(V_{1} \cap V_{4}\right) \\
& =\mathbb{R}^{4 \mid 12} \times\left(V_{2} \cap V_{3}\right),
\end{aligned}
$$

where $U_{ \pm}$and $V_{ \pm}$cover projective spaces $\mathbb{C} P^{1}=U_{+} \cup U_{-}$and $\mathbb{C} P_{*}^{1}=V_{+} \cup$ $V_{-}$parametrized by homogeneous coordinates $\left[\lambda_{\dot{\alpha}}\right]$ and $\left[\mu_{\alpha}\right]$, respectively. Recall that the manifold $\mathbb{C} P^{1} \times \mathbb{C} P_{*}^{1}$ is covered by four patches $V_{a}$ defined

\footnotetext{
${ }^{48}$ Our notation is slightly sloppy: We use the same symbol $\tau_{1}$ for maps defined on different spaces.
} 
in (7.11) with coordinates $\left(\lambda_{(a)}, \zeta_{(a)}\right)$ on $V_{a}$. The involution $\tau_{1}$ interchanges these patches as $V_{1} \leftrightarrow V_{4}, V_{2} \leftrightarrow V_{3}$ and therefore

$$
\begin{aligned}
& V_{1} \cap V_{2} \longleftrightarrow V_{4} \cap V_{3}, \quad V_{1} \cap V_{3} \longleftrightarrow V_{4} \cap V_{2}, \quad V_{1} \cap V_{4} \longleftrightarrow V_{4} \cap V_{1}, \\
& V_{2} \cap V_{3} \longleftrightarrow V_{3} \cap V_{2} .
\end{aligned}
$$

Considering $\left(\lambda_{(a)}, \zeta_{(a)}\right) \neq 0$, we impose a reality condition on the complex regular matrix-valued functions $\psi_{a}=\psi_{a}\left(x^{\alpha \dot{\alpha}}, \theta^{\alpha i}, \eta_{i}^{\dot{\alpha}}, \lambda_{(a)}, \zeta_{(a)}\right)$ by taking them depending on $\tau_{1}$-real coordinates $x^{\alpha \dot{\alpha}}, \theta^{\alpha i}, \eta_{i}^{\dot{\alpha}}$ and satisfying the equations

$$
\begin{aligned}
& \psi_{1}^{\dagger}\left(x, \theta, \eta, \frac{1}{\bar{\lambda}_{(1)}}, \frac{1}{\bar{\zeta}_{(1)}}\right)=\psi_{4}^{-1}\left(x, \theta, \eta, \lambda_{(4)}, \zeta_{(4)}\right), \\
& \psi_{2}^{\dagger}\left(x, \theta, \eta, \frac{1}{\bar{\lambda}_{(2)}}, \frac{1}{\bar{\zeta}_{(2)}}\right)=\psi_{3}^{-1}\left(x, \theta, \eta, \lambda_{(3)}, \zeta_{(3)}\right),
\end{aligned}
$$

which lead to the relations

$$
\begin{aligned}
& f_{14}^{\dagger}\left(\ldots, \frac{1}{\bar{\lambda}_{(1)}}, \frac{1}{\bar{\zeta}_{(1)}}\right)=f_{14}\left(\ldots, \lambda_{(1)}, \zeta_{(1)}\right), \\
& f_{23}^{\dagger}\left(\ldots, \frac{1}{\bar{\lambda}_{(2)}}, \frac{1}{\bar{\zeta}_{(2)}}\right)=f_{23}\left(\ldots, \lambda_{(3)}, \zeta_{(3)}\right), \\
& f_{12}^{\dagger}\left(\ldots, \frac{1}{\bar{\lambda}_{(1)}}, \frac{1}{\bar{\zeta}_{(1)}}\right)=f_{43}\left(\ldots, \lambda_{(3)}, \zeta_{(3)}\right), \\
& f_{13}^{\dagger}\left(\ldots, \frac{1}{\bar{\lambda}_{(1)}}, \frac{1}{\bar{\zeta}_{(1)}}\right)=f_{42}\left(\ldots, \lambda_{(4)}, \zeta_{(4)}\right) .
\end{aligned}
$$

Now, using the definitions (9.8)-(9.10) one can show by direct calculations that the conditions (10.13) yield antihermitean superconnections and the real $\mathcal{N}=3,4$ supermultiplet of ordinary fields.

\subsection{The Minkowskian involution $\tau_{M}$}

Let us consider the supermanifold $\mathcal{P}^{3 \mid 3} \times \mathcal{P}_{*}^{3 \mid 3}$ with homogeneous coordinates $\left[\omega^{\alpha}, \lambda_{\dot{\alpha}}, \eta_{i} ; \mu_{\alpha}, \sigma^{\dot{\alpha}}, \theta^{i}\right]$. The antiholomorphic involution

$$
\tau_{M}: \mathcal{P}^{3 \mid 3} \times \mathcal{P}_{*}^{3 \mid 3} \longrightarrow \mathcal{P}^{3 \mid 3} \times \mathcal{P}_{*}^{3 \mid 3}
$$

appropriate to Minkowski space is defined as the map (see e.g., [45])

$$
\tau_{\mathrm{M}}\left(\omega^{\alpha}, \lambda_{\dot{\alpha}}, \eta_{i} ; \mu_{\alpha}, \sigma^{\dot{\alpha}}, \theta^{i}\right)=\left(-\overline{\sigma^{\dot{\alpha}}}, \overline{\mu_{\alpha}}, \overline{\theta^{i}} ; \overline{\lambda_{\dot{\alpha}}},-\overline{\omega^{\alpha}}, \overline{\eta_{i}}\right)
$$


interchanging $\alpha$-superplanes and $\beta$-superplanes. One sees from (10.16) that the real slice in the space $\mathcal{P}^{3 \mid 3} \times \mathcal{P}_{*}^{3 \mid 3}$ is defined by the equation ${ }^{49}$

$$
\sigma^{\dot{\alpha}}=-\overline{\omega^{\alpha}}, \quad \mu_{\alpha}=\overline{\lambda_{\dot{\alpha}}}
$$

and

$$
\eta_{i}=\eta_{i}^{\dot{\alpha}} \lambda_{\dot{\alpha}}=\overline{\theta^{i}}=\overline{\theta^{\alpha i} \mu_{\alpha}}=\overline{\theta^{\alpha i}} \overline{\mu_{\alpha}} \quad \Longrightarrow \quad \eta_{i}^{\dot{\alpha}}=\overline{\theta^{\alpha i}}
$$

Finally, for coordinates $\left(x^{\alpha \dot{\alpha}}\right) \in \mathbb{C}^{4}$, we have

$$
\tau_{\mathrm{M}}\left(x^{\alpha \dot{\beta}}\right)=-\bar{x}^{\beta \dot{\alpha}},
$$

and the Minkowskian real slice $\mathbb{R}^{3,1} \subset \mathbb{C}^{4}$ is parametrized by coordinates

$$
\begin{gathered}
\left(\begin{array}{ll}
x^{1 \dot{1}} & x^{1 \dot{2}} \\
x^{2 \dot{1}} & x^{2 \dot{2}}
\end{array}\right)^{\dagger}=-\left(\begin{array}{ll}
x^{1 \dot{1}} & x^{1 \dot{2}} \\
x^{2 \dot{1}} & x^{2 \dot{2}}
\end{array}\right) \\
\Longrightarrow x^{1 \dot{1}}=-\mathrm{i}\left(x^{0}+x^{3}\right), \quad x^{1 \dot{2}}=-\mathrm{i}\left(x^{1}-\mathrm{i} x^{2}\right), \\
x^{2 \dot{1}}=-\mathrm{i}\left(x^{1}+\mathrm{i} x^{2}\right), \quad x^{2 \dot{2}}=-\mathrm{i}\left(x^{0}-x^{3}\right)
\end{gathered}
$$

with $\left(x^{0}, x^{1}, x^{2}, x^{3}\right) \in \mathbb{R}^{4}$ and (cf. (2.18))

$$
d s^{2}=\operatorname{det}\left(d x^{\alpha \dot{\alpha}}\right) \Longrightarrow g=\operatorname{diag}(-1,+1,+1,+1) .
$$

One can also introduce coordinates

$$
\tilde{x}^{\alpha \dot{\alpha}}=\mathrm{i} x^{\alpha \dot{\alpha}},
$$

obtaining a metric with signature $(1,3)$. In terms of $\tilde{x}^{\alpha \dot{\alpha}}$, imaginary units will appear in many formulæ, which is the common convention on Minkowski space with signature $(1,3)$, e.g.,

$$
D_{\alpha i}=\frac{\partial}{\partial \theta^{\alpha i}}+\mathrm{i} \bar{\theta}_{i}^{\dot{\alpha}} \frac{\partial}{\partial \tilde{x}^{\alpha \dot{\alpha}}}, \quad \tilde{x}_{\mathrm{R}}^{\alpha \dot{\alpha}}=\tilde{x}^{\alpha \dot{\alpha}}-\mathrm{i} \theta^{\alpha i} \bar{\theta}_{i}^{\dot{\alpha}},
$$

etc.

Recall that the involution $\tau_{\mathrm{M}}$ interchanges $\alpha$-superplanes and $\beta$-superplanes and therefore exchanges opposite helicity states. It might be identified with a $\mathbb{Z}_{2}$-symmetry discussed recently in the context of mirror symmetry [9] and parity invariance [15].

\footnotetext{
${ }^{49}$ Here $\alpha$ and $\dot{\alpha}$ denote the same number.
} 


\subsection{A $\tau_{\mathrm{M}}$-real twistor diagram}

Recall that $\left[\lambda_{\dot{\alpha}}\right]$ and $\left[\mu_{\alpha}\right]$ are homogeneous coordinates on two Riemann spheres and the involution $\tau_{\mathrm{M}}$ maps these spheres one into another. Moreover, fixed points of the map $\tau_{\mathrm{M}}: \mathbb{C} P^{1} \times \mathbb{C} P_{*}^{1} \rightarrow \mathbb{C} P^{1} \times \mathbb{C} P_{*}^{1}$ form the Riemann sphere

$$
\mathbb{C} P^{1}=\operatorname{diag}\left(\mathbb{C} P^{1} \times \overline{\mathbb{C} P}^{1}\right),
$$

where $\overline{\mathbb{C} P}{ }^{1}\left(=\mathbb{C} P_{*}^{1}\right)$ denotes the Riemann sphere $\mathbb{C} P^{1}$ with the opposite complex structure. Therefore, a real slice in the space $\mathcal{F}^{6 \mid 12}=\mathbb{C}^{4 \mid 12} \times \mathbb{C} P^{1} \times$ $\mathbb{C} P_{*}^{1}$ introduced in (7.15) and characterized as the fixed point set of the involution $\tau_{\mathrm{M}}$ is the space

$$
\mathcal{F}_{\tau_{\mathrm{M}}}^{6 \mid 12}:=\mathbb{R}^{4 \mid 12} \times \mathbb{C} P^{1}
$$

of real dimension $(6 \mid 12)$.

The fixed point set of the involution (10.15) is the diagonal in the space $\mathcal{P}^{3 \mid 3} \times \overline{\mathcal{P}}^{3 \mid 3}$, which can be identified with the complex supertwistor space $\mathcal{P}^{3 \mid 3}$ of real dimension $(6 \mid 6)$. This involution also picks out a real quadric $\mathcal{L}_{\tau_{\mathrm{M}}}^{5 \mid 6}$ defined by equations (7.8) and the reality conditions (10.17)-(10.20). Thus, we obtain a real version of the double fibration (7.15),

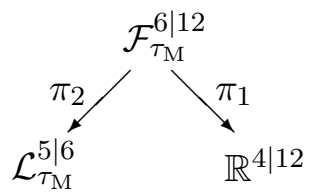

The dimensions of all spaces in this diagram are real. For imposing the reality conditions on the superconnection components, one should proceed analogously to the case of Kleinian signature. We will not discuss this here.

\section{Conclusions}

In this paper, we considered two examples of the fibration

$$
\pi: Z \longrightarrow X_{\tau},
$$

which describe self-dual and anti-self-dual $\mathcal{N}$-extended SYM theory in four real dimensions. As the supermanifold $Z$, we used the supertwistor space $\mathcal{P}^{3 \mid \mathcal{N}}=\mathbb{C} P^{3 \mid \mathcal{N}} \backslash \mathbb{C} P^{1 \mid \mathcal{N}}$ (self-dual case) and the dual supertwistor space $\mathcal{P}_{*}^{3 \mid \mathcal{N}}=\mathbb{C} P_{*}^{3 \mid \mathcal{N}} \backslash \mathbb{C} P_{*}^{1 \mid \mathcal{N}}$ (anti-self-dual case) with $0 \leq \mathcal{N} \leq 4$. As the supermanifold $X_{\tau}$, we chose the real anti-chiral superspace $\mathcal{R}_{\mathrm{R}}^{4 \mid 2 \mathcal{N}}$ (self-dual case) and the real chiral superspace $\mathcal{R}_{\mathrm{L}}^{4 \mid 2 \mathcal{N}}$ (anti-self-dual case). In both cases, we considered hCS theory on the supermanifold $Z$ and showed that, by using 
a gauge transformation on $Z$, one can bring Witten's form of the hCS field equations to the previously known constrained equations on the supercurvature field strength corresponding to $\mathcal{N}$-extended self-dual or anti-self-dual SYM theory on $\mathcal{R}_{\mathrm{R}}^{4 \mid 2 \mathcal{N}}$ or $\mathcal{R}_{\mathrm{L}}^{4 \mid 2 \mathcal{N}}$ with a metric on the body ${ }^{50}$ of signature $(4,0)$ or $(2,2)$.

Considering hCS theory on the supertwistor space $\mathcal{P}^{3 \mid \mathcal{N}}$, we gave an explicit expansion of the super gauge potential in coordinates on $\mathbb{C} P^{1} \subset$ $\mathcal{P}^{3 \mid \mathcal{N}}$ in which the equivalence of the equations of motion $\bar{\partial} \hat{\mathcal{A}}^{0,1}+\hat{\mathcal{A}}^{0,1} \wedge$ $\hat{\mathcal{A}}^{0,1}=0$ to the equations of motion of self-dual $\mathcal{N}$-extended SYM theory in four dimensions becomes manifest. All this was translated to the anti-selfdual case by using the dual supertwistor space $\mathcal{P}_{*}^{3 \mid \mathcal{N}}$.

We also considered an example of the double fibration

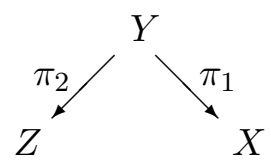

where $X$ was chosen to be the superspace $\mathbb{C}^{4 \mid 12}$ or its real version $\mathbb{R}^{4 \mid 12}$ with a metric on the body of signature $(4,0),(2,2)$ or $(3,1)$. As supermanifold $Z$, we used the quadric $\mathcal{L}^{5 \mid 6}$ in $\mathcal{P}^{3 \mid 3} \times \mathcal{P}_{*}^{3 \mid 3}$ or a real subspace of it with the real structure depending on the signature of the metric on $\mathbb{R}^{4}$. The correspondence space $Y=\mathcal{F}^{6 \mid 12}=\mathbb{C}^{4 \mid 12} \times \mathbb{C} P^{1} \times \mathbb{C} P_{*}^{1}$ was embedded as a submanifold $^{51}$ in $Z \times X$ by using the projections $\left(\pi_{1}, \pi_{2}\right)$. We showed that, by using a gauge transformation on the correspondence space, one can bring Witten's form of the hCS field equations to the well-known constraint equations on the supercurvature field strength corresponding to full $\mathcal{N}=3 \mathrm{SYM}$ theory on the superspace $\mathbb{C}^{4 \mid 12}$ or one of its real subspaces. This theory is known to be equivalent to $\mathcal{N}=4$ SYM theory, when formulated on $\mathbb{R}^{4}$.

There are a lot of open problems which deserve further study. On the field theory side, it is not clear yet how to construct an action for hCS theory on $\mathcal{L}^{5 \mid 6}$ which will correspond to the action of $\mathcal{N}=4$ SYM theory. Generalizations of the twistor correspondence and the Penrose-Ward transform to the SFT level may also be of interest. This could either be done in the setting proposed by [8], although it seems that due to the off-shell character of SFT one should employ the more general setting [10]; or one could concentrate on (an appropriate extension of) SFT for $N=2$ string theory. This theory is known to describe SDYM at tree level [50]; its SFT [51] is based on a

\footnotetext{
${ }^{50}$ Here the body is $\left(\mathbb{R}^{4}, g\right)$. See also Appendix B.

${ }^{51}$ Recall that $Y$ is fibred over $X$ with fibres $\pi_{1}^{-1}(x)$ diffeomorphic to submanifolds $\pi_{2}\left(\pi_{1}^{-1}(x)\right)$ of $Z$ and $Y$ is also fibred over $Z$ with fibres $\pi_{2}^{-1}(z)$ which are diffeomorphic to submanifolds $\pi_{1}\left(\pi_{2}^{-1}(z)\right)$ of $X$, i.e., $Y \hookrightarrow Z \times X$.
} 
description of $N=2$ string theory as a topological $N=4$ theory [52]. This description contains twistors from the outset. The coordinate $\lambda \in \mathbb{C} P^{1}$, the linear system, integrability and the solution of the equations of motion by twistor methods were incorporated into the $N=2$ open SFT in $[53,54]$. However, this theory reproduces only classical bosonic SDYM theory, its symmetries and integrability properties $[53,55,56]$. Following various proposals, e.g. $[4,35,57,58]$ (see also references therein), one can extend it to be spacetime supersymmetric. This is believed to lead to an explicit relation between the supersymmetric extension of $N=4$ topological string theory and the $N=2$ topological string (B-type) [5], but the picture is far from being complete.

\section{Acknowledgments}

We are grateful to O. Lechtenfeld, S. Uhlmann and M. Wolf for many useful comments. This work was partially supported by the Deutsche Forschungsgemeinschaft (DFG).

\section{Appendix A Dictionary: homogeneous $\longleftrightarrow$ inhomogeneous coordinates}

The sphere $S^{2}$ is diffeomorphic to the complex projective space $\mathbb{C} P^{1}$. This space can be parametrized globally by complex homogeneous coordinates $\lambda_{i}$ and $\lambda_{\dot{2}}$ which are not simultaneously zero (in projective spaces, the origin is excluded). So, the Riemann sphere $\mathbb{C} P^{1}$ can be covered by two coordinate patches

$$
U_{+}=\left\{\left[\lambda_{\dot{1}}, \lambda_{\dot{2}}\right] \mid \lambda_{\dot{1}} \neq 0\right\} \quad \text { and } \quad U_{-}=\left\{\left[\lambda_{\dot{1}}, \lambda_{\dot{2}}\right] \mid \lambda_{\dot{2}} \neq 0\right\},
$$

with coordinates

$$
\lambda_{+}:=\frac{\lambda_{\dot{2}}}{\lambda_{\dot{1}}} \quad \text { on } \quad U_{+} \quad \text { and } \quad \lambda_{-}:=\frac{\lambda_{\dot{1}}}{\lambda_{\dot{2}}} \quad \text { on } \quad U_{-} .
$$

On the intersection $U_{+} \cap U_{-}$, we get $\lambda_{+}=1 / \lambda_{-}$.

A global section of the holomorphic line bundle ${ }^{52} \mathcal{O}(n)$ over $\mathbb{C} P^{1}$ exists only for $n \geq 0$. Over $U_{ \pm}$, it is represented by a polynomial $p_{ \pm}^{(n)}$ of degree $n$ in the coordinates $\lambda_{ \pm}$with $p_{+}^{(n)}=\lambda_{+}^{n} p_{-}^{(n)}$ on $U_{+} \cap U_{-}$. The explicit expansion

\footnotetext{
${ }^{52}$ See Appendix B.
} 
will look like

$$
\begin{aligned}
& p_{+}^{(n)}=a_{0}+a_{1} \lambda_{+}+a_{2} \lambda_{+}^{2}+\cdots+a_{n} \lambda_{+}^{n} \text { and } \\
& p_{-}^{(n)}=a_{0} \lambda_{-}^{n}+\cdots+a_{n-2} \lambda_{-}^{2}+a_{n-1} \lambda_{-}+a_{n}
\end{aligned}
$$

and, multiplying the expansion in $\lambda_{+}$by $\lambda_{\dot{1}}^{n}$ (or the expansion in $\lambda_{-}$by $\lambda_{\dot{2}}^{n}$ ), one obtains a homogeneous polynomial of degree $n$ :

$$
a_{0} \lambda_{\dot{1}}^{n}+a_{1} \lambda_{\dot{1}}^{n-1} \lambda_{\dot{2}}+\cdots+a_{n-1} \lambda_{\dot{1}} \lambda_{\dot{2}}^{n-1}+a_{n} \lambda_{\dot{2}}^{n}=: Q^{\dot{\alpha}_{1} \ldots \dot{\alpha}_{n}} \lambda_{\dot{\alpha}_{1}} \ldots \lambda_{\dot{\alpha}_{n}} .
$$

Now let us consider the expansions (5.33) and (5.34) of the super gauge potentials of hCS theory on the supertwistor space. We get the following list of objects:

$$
\begin{array}{lcl}
\eta_{i}^{+} & \mathcal{O}(1) & \eta_{i}=\lambda_{i} \eta_{i}^{+} \\
\gamma_{+} & \mathcal{O}(-1) \otimes \overline{\mathcal{O}}(-1) & \gamma=\frac{1}{\lambda_{i} \bar{\lambda}_{\dot{1}}} \gamma_{+}\left(=\frac{1}{\lambda^{\dot{\alpha}} \hat{\lambda}_{\dot{\alpha}}}\right) \\
\hat{\mathcal{A}}_{\alpha}^{+} & \mathcal{O}(1) & \hat{\mathcal{A}}_{\alpha}=\lambda_{\dot{1}} \hat{\mathcal{A}}_{\alpha}^{+} \\
\hat{\mathcal{A}}_{\bar{\lambda}_{+}} & \overline{\mathcal{O}}(-2) & \hat{\mathcal{A}}_{3}=\frac{1}{\bar{\lambda}_{\dot{1}} \bar{\lambda}_{\dot{1}}} \hat{\mathcal{A}}_{\bar{\lambda}_{+}} .
\end{array}
$$

This implies the following expansions in homogeneous coordinates (cf. (5.33) and (5.34)):

$$
\begin{aligned}
\hat{\mathcal{A}}_{\alpha}= & \lambda^{\dot{\alpha}} A_{\alpha \dot{\alpha}}\left(x_{\mathrm{R}}\right)+\eta_{i} \chi_{\alpha}^{i}\left(x_{\mathrm{R}}\right)+\gamma \frac{1}{2 !} \eta_{i} \eta_{j} \hat{\lambda}^{\dot{\alpha}} \phi_{\alpha \dot{\alpha}}^{i j}\left(x_{\mathrm{R}}\right) \\
& +\gamma^{2} \frac{1}{3 !} \eta_{i} \eta_{j} \eta_{k} \hat{\lambda}^{\dot{\alpha}} \hat{\lambda}^{\dot{\beta}} \tilde{\chi}_{\alpha \dot{\alpha} \dot{\beta}}^{i j k}\left(x_{\mathrm{R}}\right) \\
& +\gamma^{3} \frac{1}{4 !} \eta_{i} \eta_{j} \eta_{k} \eta_{l} \hat{\lambda}^{\dot{\alpha}} \hat{\lambda}^{\dot{\beta}} \hat{\lambda}^{\dot{\gamma}} G_{\alpha \dot{\alpha} \dot{\beta} \dot{\gamma}}^{i j k l}\left(x_{\mathrm{R}}\right), \\
\hat{\mathcal{A}}_{3}= & \gamma^{2} \frac{1}{2 !} \eta_{i} \eta_{j} \phi^{i j}\left(x_{\mathrm{R}}\right)+\gamma^{3} \frac{1}{3 !} \eta_{i} \eta_{j} \eta_{k} \hat{\lambda}^{\dot{\alpha}} \tilde{\chi}_{\dot{\alpha}}^{i j k}\left(x_{\mathrm{R}}\right) \\
& +\gamma^{4} \frac{1}{4 !} \eta_{i} \eta_{j} \eta_{k} \eta_{l} \hat{\lambda}^{\dot{\alpha}} \hat{\lambda}^{\dot{\beta}} G_{\dot{\alpha} \dot{\beta}}^{i j k l}\left(x_{\mathrm{R}}\right) .
\end{aligned}
$$

For rewriting the equations of motion in terms of this gauge potential, we also need to rewrite the vector fields (4.19) and (4.33) in homogeneous coordinates. The vector fields along the fibres are easily rewritten, analogously to the corresponding components of the gauge potential. The vector field on the sphere can be calculated by considering $\hat{\mathcal{A}}_{\bar{\lambda}_{+}} d \bar{\lambda}_{+}=\hat{\mathcal{A}}_{3} \bar{\Theta}^{3}$. This implies $\bar{\Theta}^{3}=\bar{\lambda}_{\dot{1}} d \bar{\lambda}_{\dot{2}}-\bar{\lambda}_{\dot{2}} d \bar{\lambda}_{\dot{1}}$, which has a dual vector field $\bar{V}_{3}$ defined by $\left.\bar{V}_{3}\right\lrcorner \bar{\Theta}^{3}=1$. 
Altogether, we obtain the basis

$$
\bar{V}_{\alpha}=\lambda^{\dot{\alpha}} \frac{\partial}{\partial x_{\mathrm{R}}^{\alpha \dot{\alpha}}} \quad \text { and } \quad \bar{V}_{3}=-\gamma \lambda^{\dot{\alpha}} \frac{\partial}{\partial \hat{\lambda}^{\dot{\alpha}}} .
$$

The field equations (5.31) and (5.32) now take the form

$$
\begin{aligned}
\bar{V}_{\alpha} \hat{\mathcal{A}}_{\beta}-\bar{V}_{\beta} \hat{\mathcal{A}}_{\alpha}+\left[\hat{\mathcal{A}}_{\alpha}, \hat{\mathcal{A}}_{\beta}\right]=0, \\
\bar{V}_{3} \hat{\mathcal{A}}_{\alpha}-\bar{V}_{\alpha} \hat{\mathcal{A}}_{3}+\left[\hat{\mathcal{A}}_{3}, \hat{\mathcal{A}}_{\alpha}\right]=0
\end{aligned}
$$

and yield the same equations (5.17) for the physical fields.

\section{Appendix B Some mathematical definitions}

\section{B.1 Interior product}

For the interior product of a vector $V$ with a one-form $A$, we use the notation $V\lrcorner A:=\langle V, A\rangle$. A second common notation for this product is $\mathrm{i}_{V} A$.

\section{B.2 Holomorphic line bundles}

Given the Riemann sphere $\mathbb{C} P^{1} \cong S^{2}$ with standard patches $U_{+}$and $U_{-}$and coordinates $\lambda_{ \pm}$on the corresponding patches and $\lambda_{ \pm}=1 / \lambda_{\mp}$ on $U_{+} \cap U_{-}$, the holomorphic line bundle $\mathcal{O}(n)$ is defined by its transition function $z_{+}=$ $\lambda_{+}^{n} z_{-}$, where $z_{ \pm}$are complex coordinates on fibres over $U_{ \pm}$. For $n \geq 0$, global sections of the bundle $\mathcal{O}(n)$ are polynomials of degree $n$ in the coordinates $\lambda_{ \pm}$and homogeneous polynomials of degree $n$ in homogeneous coordinates (see also Appendix A). The $\mathcal{O}(n)$ line bundle has first Chern number $n$. The complex conjugate bundle to $\mathcal{O}(n)$ is denoted by $\overline{\mathcal{O}}(n)$. Its sections have transition functions $\bar{\lambda}_{+}^{n}: \bar{z}_{+}=\bar{\lambda}_{+}^{n} \bar{z}_{-}$.

\section{B.3 Spinor conventions}

All objects with space-time indices are rewritten in spinor notation by $x^{\alpha \dot{\alpha}}=\sigma_{\mu}^{\alpha \dot{\alpha}} x^{\mu}$, etc., where the sigma-matrices are determined by the metric under consideration. The homogeneous coordinates $\lambda_{i}$ and $\lambda_{\dot{2}}$ for a point in $\mathbb{C} P^{1}$ are regarded as components of a complex commuting spinors. Their indices are raised and lowered with the antisymmetric $\varepsilon$-tensors. We use the convention $\varepsilon_{12}=\varepsilon_{\dot{1} \dot{2}}=-\varepsilon^{12}=-\varepsilon^{\dot{i} \dot{2}}=1$, implying $\varepsilon^{\alpha \beta} \varepsilon_{\beta \gamma}=\delta_{\gamma}^{\alpha}$. The complex conjugate is obtained by conjugating the components of the spinor. 
A second antilinear conjugation, denoted by $\hat{\imath}$ is performed for different types of spinors as

$$
\left(\hat{\mu}_{\alpha}\right):=C\left(\bar{\mu}_{\alpha}\right), \quad\left(\hat{\mu}^{\alpha}\right):=C\left(\bar{\mu}^{\alpha}\right), \quad\left(\hat{\lambda}_{\dot{\alpha}}\right):=C\left(\bar{\lambda}_{\dot{\alpha}}\right) \quad \text { and } \quad\left(\hat{\lambda}^{\dot{\alpha}}\right):=C\left(\bar{\lambda}^{\dot{\alpha}}\right),
$$

where the $2 \times 2$-matrix $C$ is given by

$$
C=\left(\begin{array}{ll}
0 & \varepsilon \\
1 & 0
\end{array}\right) .
$$

The conventions for Graßmann variables are discussed in the text around (4.21)-(4.27) and (10.16). These imply in particular that

$$
\overline{\frac{\partial}{\partial \xi}}=\frac{\partial}{\partial \bar{\xi}} \text {. }
$$

Furthermore, we adopt the following convention for the conjugation of products of Graßmann variables and supernumbers in general:

$$
\tau\left(\xi^{1} \xi^{2}\right)=\tau\left(\xi^{1}\right) \tau\left(\xi^{2}\right) \text { and } \tau\left(z^{1} z^{2}\right)=\tau\left(z^{1}\right) \tau\left(z^{2}\right) .
$$

With this choice, products of two real objects will be real. Note that this is not the common convention used for supersymmetry in Minkowski space, and here, we define $\tau_{\mathrm{M}}\left(\xi^{1} \xi^{2}\right)=\tau_{\mathrm{M}}\left(\xi^{2}\right) \tau_{\mathrm{M}}\left(\xi^{1}\right)$. A more detailed discussion can be found in [59].

\section{B.4 Flag manifolds}

Complex flag manifolds are a major tool in the context of twistors and the Penrose-Ward correspondence. They can be considered as a generalization of projective spaces and Graßmann manifolds. An $r$-tuple of vector spaces $\left(L_{1}, \ldots, L_{r}\right)$ of dimensions $\operatorname{dim}_{\mathbb{C}} L_{i}=d_{i}$ with $L_{1} \subset \cdots \subset L_{r} \subset \mathbb{C}^{n}$ and $0<$ $d_{0}<\cdots<d_{r}<n$ is called a flag in $\mathbb{C}^{n}$. A (complex) flag manifold is the (compact) space

$$
F_{d_{1} \cdots d_{r}}:=\left\{\text { all flags }\left(L_{1}, \ldots, L_{r}\right) \text { with } \operatorname{dim}_{\mathbb{C}} L_{i}=d_{i}, i=1, \ldots, r\right\} .
$$

Simple examples are $F_{1}=\mathbb{C} P^{n-1}$ and $F_{k}=G_{k, n}(\mathbb{C})$.

To see how flag manifolds naturally arise, consider the following reformulation of the (bosonic part of the) discussion following (2.8). We fix the full space to be $\mathbb{C}^{4}$. Then we can establish the following double fibration:

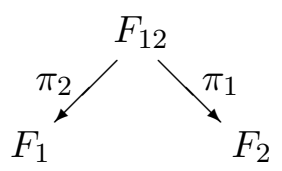

Let $\left(L_{1}, L_{2}\right)$ be an element of $F_{12}$, i.e., $\operatorname{dim}_{\mathbb{C}} L_{1}=1, \operatorname{dim}_{\mathbb{C}} L_{2}=2$ and $L_{1} \subset$ $L_{2}$. Thus, $F_{12}$ fibres over $F_{2}$ with $\mathbb{C} P^{1}$ as a typical fibre, which parametrizes 
the freedom to choose a complex one-dimensional subspace in a complex twodimensional vector space. The projections are defined as $\pi_{2}\left(L_{1}, L_{2}\right)=L_{1}$ and $\pi_{1}\left(L_{1}, L_{2}\right)=L_{2}$. The full connection to (2.8) becomes obvious, when we note that $F_{1}=\mathbb{C} P^{3}=\mathcal{P}^{3} \cup \mathbb{C} P^{1}$ and that $F_{2}=G_{2,4}(\mathbb{C})$ is the complexified and compactified version of $\mathbb{R}^{4}$. The advantage of the formulation in terms of flag manifolds is related to the fact that the projections are immediately clear: one has to shorten the flags to suit the structure of the flags of the base space.

The compactified version of the "dual" fibration (6.10) is

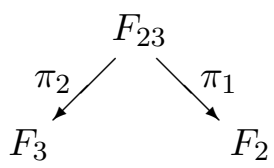

where $F_{3}$ is the space of hyperplanes in $\mathbb{C}^{4}$. This space is naturally dual to the space of lines, as every hyperplane is fixed by a vector orthogonal to the elements of the hyperplane. Therefore, we have $F_{3}=F_{1}^{*}=\mathbb{C} P_{*}^{3} \supset \mathcal{P}_{*}^{3}$.

Also, the third double fibration (7.15), which we used in the case of full $\mathcal{N}=3 \mathrm{SYM}$, is a restricted version of the diagram

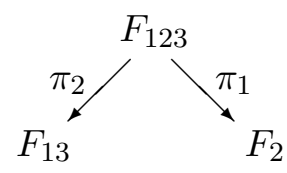

where $F_{2}=G_{2,4}(\mathbb{C})$ is again the complexified and compactified version of $\mathbb{R}^{4}$. The flag manifold $F_{13}$ is topologically the zero locus of a quadric in $\mathbb{C} P^{3} \times$ $\mathbb{C} P_{*}^{3}$. For further details and the $\mathbb{Z}_{2}$-graded generalization, see e.g. $[22,38]$.

\section{B.5 Supermanifolds and CY supermanifolds}

The space $\mathbb{R}^{r \mid s}$ is described by coordinates $x^{i}$ and $\theta^{j}$ with $1 \leq i \leq r, 1 \leq$ $j \leq s$, where the $\theta^{j}$ are real Graßmann variables satisfying the algebra $\left\{\theta^{j}, \theta^{k}\right\}=0$. The superspace $\mathbb{C}^{r \mid s}$ is defined analogously, with complex coordinates: $\bar{x}^{i} \neq x^{i}, \bar{\theta}^{j} \neq \theta^{j}$. For our considerations, a supermanifold is defined to be a topological space which is locally diffeomorphic to $\mathbb{R}^{r \mid s}$ or $\mathbb{C}^{r \mid s}$.

A supermanifold contains a purely bosonic part (the "body") which is parametrized in terms of bosonic coordinates. The body of a supermanifold is a real or complex manifold by itself. The $\mathbb{Z}_{2}$-grading of the superspace used for parametrizing the supermanifold induces a grading on the ring of functions on the supermanifold. For objects like subspaces, forms, etc. which 
come with a dimension, a degree, etc., we use the notation $(i \mid j)$, where $i$ and $j$ denote the bosonic and fermionic parts, respectively.

We further introduce the parity-changing operator $\Pi$ which, when acting on a fibre bundle, changes the parity of the fibre coordinates. For example, $\Pi \mathcal{O}(n) \rightarrow \mathbb{C} P^{1}$ is parametrized by complex variables $\lambda_{ \pm}$and Graßmann variables $\theta_{ \pm}$with $\theta_{+}=\lambda_{+}^{n} \theta_{-}$on $U_{+} \cap U_{-}$.

For a more extensive discussion of supermanifolds, see [59] and references therein.

CY manifolds are manifolds with vanishing first Chern class which implies the existence of a globally well-defined holomorphic volume form. For our purposes, we define a $C Y$ supermanifold to be a supermanifold with a globally defined holomorphic volume form. Note that the body of a super-CY is not a $\mathrm{CY}$, in general.

In the purely bosonic case, the 3 -fold $\mathcal{O}(m) \oplus \mathcal{O}(n) \rightarrow \mathbb{C} P^{1}$ with coordinates $z_{ \pm}^{1}, z_{ \pm}^{2}, \lambda_{ \pm}$is a CY if, and only if, $m+n=-2$, and a volume form is then given by $\Omega_{ \pm}^{3,0}= \pm d z_{ \pm}^{1} \wedge d z_{ \pm}^{2} \wedge d \lambda_{ \pm}$. In the super case, the fermionic coordinates can also be assigned to some line bundle, but because the Berezinian (i.e., the fermionic Jacobi determinant) enters as an inverse in the integration, a fermionic coordinate living in $\mathcal{O}(n)$ will contribute $-n$ to the overall first Chern number. Thus, the bundle

$$
\mathcal{P}^{3 \mid 4}=\mathcal{O}(1) \oplus \mathcal{O}(1) \oplus \Pi \mathcal{O}(1) \oplus \Pi \mathcal{O}(1) \oplus \Pi \mathcal{O}(1) \oplus \Pi \mathcal{O}(1) \longrightarrow \mathbb{C} P^{1},
$$

is a CY supermanifold. Its holomorphic volume form is given by $\hat{\Omega}_{ \pm}^{3,0 \mid 4,0}=$ $\pm d z_{ \pm}^{1} \wedge d z_{ \pm}^{2} \wedge d \lambda_{ \pm} d \theta_{ \pm}^{1} d \theta_{ \pm}^{2} d \theta_{ \pm}^{3} d \theta_{ \pm}^{4}$, where $z_{ \pm}^{i}$ and $\theta_{ \pm}^{j}$ are coordinates of the bosonic and fermionic line bundles, respectively. The body of this supermanifold is $\mathcal{O}(1) \oplus \mathcal{O}(1) \rightarrow \mathbb{C} P^{1}$ and it is obviously not a $\mathrm{CY}$ manifold.

\section{Appendix C The twistor geometry in the Kleinian case $\varepsilon=+1$}

As mentioned several times in the text, one should consider hCS theory on domains $\hat{\mathcal{U}}_{ \pm}$of the supertwistor space $\mathcal{P}^{3 \mid \mathcal{N}}$ for which $\left|\lambda_{ \pm}\right| \neq 1$ when working in the Kleinian case, i.e., when using the reality conditions obtained from 
the involution ${ }^{53} \tau_{1}$. In this and the following appendix, we will discuss this aspect in more detail.

Let us start from the double fibration (4.6),

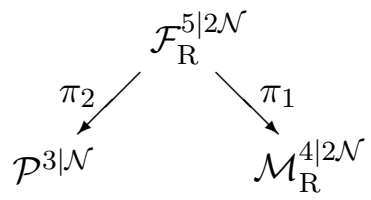

which describes the complex supertwistor correspondence for $0 \leq \mathcal{N} \leq 4$. As before, we have complex coordinates $\left(z_{ \pm}^{\alpha}, \lambda_{ \pm}, \eta_{i}^{ \pm}\right)$on the patches $\hat{\mathcal{U}}_{ \pm}$which cover $\mathcal{P}^{3 \mid \mathcal{N}}$ and $\left(x_{\mathrm{R}}^{\alpha \dot{\alpha}}, \lambda_{\dot{\alpha}}^{ \pm}, \eta_{i}^{\dot{\alpha}}\right)$ on $\mathcal{F}_{\mathrm{R}}^{5 \mid 2 \mathcal{N}}$. The projection $\pi_{1}$ is the trivial projection $\pi_{1}\left(x_{\mathrm{R}}^{\alpha \dot{\alpha}}, \lambda_{\dot{\alpha}}^{ \pm}, \eta_{i}^{\dot{\alpha}}\right)=\left(x_{\mathrm{R}}^{\alpha \dot{\alpha}}, \eta_{i}^{\dot{\alpha}}\right)$ and the projection $\pi_{2}$ is given by the formulæ

$$
\begin{gathered}
z_{ \pm}^{\alpha}=x_{\mathrm{R}}^{\alpha \dot{\alpha}} \lambda_{\dot{\alpha}}^{ \pm}, \quad \lambda_{\dot{\alpha}}^{ \pm}=\lambda_{\dot{\alpha}}^{ \pm} \quad \text { and } \quad \eta_{i}^{ \pm}=\eta_{i}^{\dot{\alpha}} \lambda_{\dot{\alpha}}^{ \pm} \quad \text { with } \quad\left(\lambda_{\dot{\alpha}}^{+}\right)=\left(\begin{array}{c}
1 \\
\lambda_{+}
\end{array}\right), \\
\left(\lambda_{\dot{\alpha}}^{-}\right)=\left(\begin{array}{c}
\lambda_{-} \\
1
\end{array}\right) .
\end{gathered}
$$

The action of the involution $\tau_{1}$ on the coordinates of $\mathcal{P}^{3 \mid \mathcal{N}}$ is given by formulæ (2.12) together with $\tau_{1}\left(\eta_{i}^{ \pm}\right)=\bar{\eta}_{i}^{ \pm} / \bar{\lambda}_{ \pm}$. It yields the reality conditions

$$
z_{ \pm}^{2}=\frac{\bar{z}_{ \pm}^{1}}{\bar{\lambda}_{ \pm}}, \quad \lambda_{ \pm}=\frac{1}{\bar{\lambda}_{ \pm}} \quad \text { and } \quad \eta_{i}^{ \pm}=\frac{\bar{\eta}_{i}^{ \pm}}{\bar{\lambda}_{ \pm}}
$$

on $\mathcal{P}^{3 \mid \mathcal{N}}$, which imply

$$
x_{\mathrm{R}}^{2 \dot{2}}=\bar{x}_{\mathrm{R}}^{1 \dot{1}}, \quad x_{\mathrm{R}}^{2 \dot{1}}=\bar{x}_{\mathrm{R}}^{1 \dot{2}} \quad \text { and } \quad \eta_{i}^{\dot{2}}=\bar{\eta}_{i}^{\dot{1}}
$$

on $\mathcal{M}_{\mathrm{R}}^{4 \mid 2 \mathcal{N}}$ and $\mathcal{F}_{\mathrm{R}}^{5 \mid 2 \mathcal{N}}=\mathcal{M}_{\mathrm{R}}^{4 \mid 2 \mathcal{N}} \times \mathbb{C} P^{1}$.

The set of fixed points under this involution ${ }^{54}$ of the spaces contained in the double fibration (C.1) form real subsets $\mathcal{T}^{3 \mid \mathcal{N}} \subset \mathcal{P}^{3 \mid \mathcal{N}}, \mathcal{R}_{\mathrm{R}}^{4 \mid 2 \mathcal{N}} \subset \mathcal{M}_{\mathrm{R}}^{4 \mid 2 \mathcal{N}}$ and $\mathcal{R}_{\mathrm{R}}^{4 \mid 2 \mathcal{N}} \times S^{1} \subset \mathcal{F}_{\mathrm{R}}^{5 \mid 2 \mathcal{N}}$. Recall that the body $\mathcal{T}^{3}$ of the supermanifold ${ }^{55}$

\footnotetext{
${ }^{53}$ For $\tau_{0}$, the description is similar and for that reason we focus on $\tau_{1}$. Note, however, that the $\mathbb{C} P^{1}$ embedded in the twistor spaces reduces to different $S^{1}$ s: for $\tau_{1}$, the constraint is $\lambda_{ \pm}=\bar{\lambda}_{ \pm}^{-1}$ and for $\tau_{0}$ we have $\lambda_{ \pm}=\bar{\lambda}_{ \pm}$.

${ }^{54}$ Although $\tau_{1}$ was defined on $\mathcal{P}^{3 \mid \mathcal{N}}$, it induces an involution on $\mathcal{F}_{\mathrm{R}}^{5 \mid 2 \mathcal{N}}$ which we will denote by the same symbol in the following.

${ }^{55}$ For $\mathcal{N}=4, \mathcal{T}^{3 \mid \mathcal{N}}$ has a globally defined real volume form invariant under rescaling of homogeneous coordinates.
} 
$\mathcal{T}^{3 \mid \mathcal{N}}$ is diffeomorphic to the space $\mathbb{R} P^{3} \backslash \mathbb{R} P^{1}$ (cf. (2.14)) fibred over $S^{1} \cong$ $\mathbb{R} P^{1} \subset \mathbb{C} P^{1}$. Thus, we obtain the real version

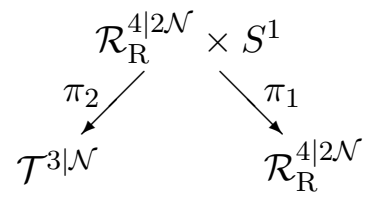

of the double fibration (C.1). Here, $\pi_{1}$ is again the trivial projection and $\pi_{2}$ is given by equations (C.2)-(C.4) with $\left|\lambda_{ \pm}\right|=1$.

The tangent spaces to the (real) $(2 \mid \mathcal{N})$-dimensional leaves of the fibration $\pi_{2}$ in (C.5) are spanned by the vector fields

$$
v_{\alpha}^{+}:=\lambda_{+}^{\dot{\alpha}} \frac{\partial}{\partial x_{\mathrm{R}}^{\alpha \dot{\alpha}}} \quad \text { and } \quad v_{+}^{i}:=\lambda_{+}^{\dot{\alpha}} \frac{\partial}{\partial \eta_{i}^{\dot{\alpha}}},
$$

which satisfy the reality conditions

$$
v_{2}^{+}=-\lambda_{+} \bar{v}_{1}^{+} \quad \text { and } \quad v_{+}^{i}=-\lambda_{+} \bar{v}_{+}^{i}
$$

where $\left|\lambda_{+}\right|=1$. Equivalently, one could also use the vector fields

$v_{\alpha}^{-}:=\lambda_{-}^{\dot{\alpha}} \frac{\partial}{\partial x_{\mathrm{R}}^{\alpha \dot{\alpha}}}=\lambda_{-} v_{\alpha}^{+} \quad$ and $\quad v_{-}^{i}:=\lambda_{-}^{\dot{\alpha}} \frac{\partial}{\partial \eta_{i}^{\dot{\alpha}}}=\lambda_{-} v_{+}^{i}$ with $\lambda_{-}=\frac{1}{\lambda_{+}}=\bar{\lambda}_{+} \cdot$

The vector fields (C.6) and (C.8) are the restrictions of the vector fields $\bar{V}_{\alpha}^{ \pm}$ and $\bar{\partial}_{ \pm}^{i}$ to $\left|\lambda_{ \pm}\right|=1$.

Consider now the double fibration

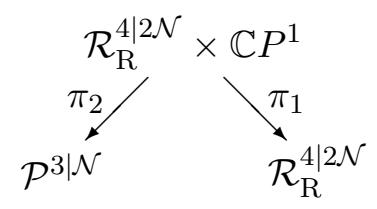

where the map $\pi_{2}$ is defined by the formulæ (C.2) with $x_{\mathrm{R}}^{\alpha \dot{\alpha}}, \eta_{i}^{\dot{\alpha}}$ satisfying the reality condition (C.4) and complex $\lambda_{\dot{\alpha}}^{ \pm}$. For Kleinian signature $(--++)$, we have the local isomorphism $\mathrm{SO}(2,2) \cong \mathrm{SU}(1,1) \times \mathrm{SU}(1,1)$ and under the action of the group $\mathrm{SU}(1,1)$, the Riemann sphere $\mathbb{C} P^{1}$ of projective spinors decomposes into the disjoint union $\mathbb{C} P^{1}=H_{+}^{2} \cup S^{1} \cup H_{-}^{2}$ of three orbits. Here, $H^{2}=H_{+}^{2} \cup H_{-}^{2}$ is the two-sheeted hyperboloid with the boundary $S^{1}$ which is stable under the involution $\tau_{1}: \lambda_{ \pm} \mapsto \bar{\lambda}_{ \pm}^{-1}$. We have $\tau_{1}\left(S^{1}\right)=S^{1}$ and $\tau_{1}\left(H_{ \pm}^{2}\right)=H_{\mp}^{2}$. This decomposition can be carried over to the spaces in 
the double fibration (C.9). We have

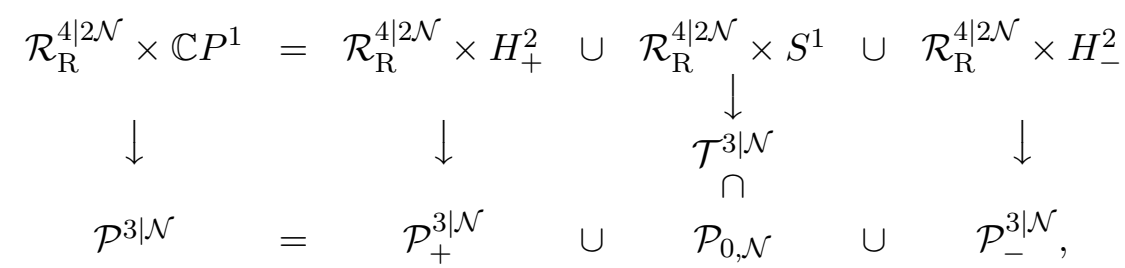

where $\downarrow$ symbolizes the projection $\pi_{2}$. Here, $\mathcal{P}_{+}^{3 \mid \mathcal{N}}, \mathcal{P}_{0, \mathcal{N}}$ and $\mathcal{P}_{-}^{3 \mid \mathcal{N}}$ are restrictions of the complex vector bundle $\mathcal{P}^{3 \mid \mathcal{N}} \rightarrow \mathbb{C} P^{1}$ to $H_{+}^{2}, S^{1}$ and $H_{-}^{2}$, respectively, and thus $\mathcal{P}_{0, \mathcal{N}}:=\left.\mathcal{P}^{3 \mid \mathcal{N}}\right|_{\lambda_{ \pm} \mid=1}$ is the common boundary of the spaces $\mathcal{P}_{+}^{3 \mid \mathcal{N}}$ and $\mathcal{P}_{-}^{3 \mid \mathcal{N}}$. Note that restricting the maps $\pi_{1}$ and $\pi_{2}$ in (C.9) to the space $\mathcal{R}_{\mathrm{R}}^{4 \mid 2 \mathcal{N}} \times S^{1}$, we obtain the real double fibration (C.5).

The map $\pi_{2}$ in (C.9) restricted to the space $\mathcal{R}_{\mathrm{R}}^{4 \mid 2 \mathcal{N}} \times H^{2}$ is a smooth bijection (diffeomorphism) of $\mathcal{R}_{\mathrm{R}}^{4 \mid 2 \mathcal{N}} \times H^{2}$ onto $\tilde{\mathcal{P}}^{3 \mid \mathcal{N}}:=\mathcal{P}^{3 \mid \mathcal{N}} \backslash \mathcal{P}_{0, \mathcal{N}}=$ $\mathcal{P}_{+}^{3 \mid \mathcal{N}} \cup \mathcal{P}_{-}^{3 \mid \mathcal{N}}$ which is defined by the formulæ (C.2) with $\left|\lambda_{ \pm}\right| \neq 1$ and $x_{\mathrm{R}}^{\alpha \dot{\alpha}}$, $\eta_{i}^{\dot{\alpha}}$ subject to (C.4). Its inverse is given by

$$
\begin{array}{r}
x_{\mathrm{R}}^{1 \mathrm{i}}=\frac{z_{+}^{1}-z_{+}^{3} \bar{z}_{+}^{2}}{1-z_{+}^{3} \bar{z}_{+}^{3}}=\frac{\bar{z}_{-}^{2}-\bar{z}_{-}^{3} z_{-}^{1}}{1-z_{-}^{3} \bar{z}_{-}^{3}}, \quad x_{\mathrm{R}}^{2 \dot{1}}=\frac{z_{+}^{2}-z_{+}^{3} \bar{z}_{+}^{1}}{1-z_{+}^{3} \bar{z}_{+}^{3}}=\frac{\bar{z}_{-}^{1}-\bar{z}_{-}^{3} z_{-}^{2}}{1-z_{-}^{3} \bar{z}_{-}^{3}}, \\
\eta_{i}^{\dot{1}}=\frac{\eta_{i}^{+}-z_{+}^{3} \bar{\eta}_{i}^{+}}{1-z_{+}^{3} \bar{z}_{+}^{3}}=\frac{\bar{\eta}_{i}^{-}-\bar{z}_{-}^{3} \eta_{i}^{-}}{1-z_{-}^{3} \bar{z}_{-}^{3}}, \quad \lambda_{ \pm}=z_{ \pm}^{3}
\end{array}
$$

and $x_{\mathrm{R}}^{2 \dot{2}}, x_{\mathrm{R}}^{1 \dot{2}}$ and $\eta_{i}^{\dot{2}}$ fixed by (C.4). Due to this diffeomorphism, the diagram (C.9) with the maps $\pi_{1}$ and $\pi_{2}$ restricted to $\mathcal{R}_{\mathrm{R}}^{4 \mid 2 \mathcal{N}} \times H^{2}$ becomes a nonholomorphic fibration

$$
\tilde{\mathcal{P}}^{3 \mid \mathcal{N}} \longrightarrow \mathcal{R}_{\mathrm{R}}^{4 \mid 2 \mathcal{N}}
$$

and on $\tilde{\mathcal{P}}^{3 \mid \mathcal{N}}$, one can use either set of coordinates $\left(z_{ \pm}^{\alpha}, \lambda_{ \pm}, \eta_{i}^{ \pm}\right)$and $\left(x_{\mathrm{R}}^{\alpha \dot{\alpha}}, \lambda_{ \pm}, \eta_{i}^{\dot{\alpha}}\right)$.

For the dual supertwistor space $\mathcal{P}_{*}^{3 \mid \mathcal{N}}$, the discussion follows along the same lines. One merely replaces the coordinates $\left(z_{ \pm}^{\alpha}, \lambda_{ \pm}, \eta_{i}^{ \pm}\right)$of $\mathcal{P}^{3 \mid \mathcal{N}}$ with the coordinates $\left(w_{ \pm}^{\dot{\alpha}}, \mu_{ \pm}, \theta_{ \pm}^{i}\right)$ of $\mathcal{P}_{*}^{3 \mid \mathcal{N}}$ and the moduli $\left(x_{\mathrm{R}}^{\alpha \dot{\alpha}}, \eta_{i}^{\dot{\alpha}}\right) \in \mathcal{M}_{\mathrm{R}}^{4 \mid 2 \mathcal{N}}$ with the moduli $\left(x_{\mathrm{L}}^{\alpha \dot{\alpha}}, \theta^{\alpha i}\right) \in \mathcal{M}_{\mathrm{L}}^{4 \mid 2 \mathcal{N}}$. Considering then the set of fixed points of the involution $\tau_{1}$ as done above leads to fibrations similar to (C.5), (C.9) and (C.12). 


\section{Appendix D Comments on hCS theory in the Kleinian case $\varepsilon=+1$}

In the case of the real structure $\tau_{1}$, i.e., $\varepsilon=+1$, which yields Kleinian signature $(2,2)$ on $\mathbb{R}^{4}$, we always discussed hCS theory on $\tilde{\mathcal{P}}^{3 \mid \mathcal{N}}$ in the text. This is due to a peculiarity of the Penrose-Ward correspondence in this case which we now discuss more explicitly.

Consider the real supertwistor space $\mathcal{T}^{3 \mid \mathcal{N}} \subset \mathcal{P}^{3 \mid \mathcal{N}}$ and a real-analytic function $f_{+-}^{\tau}: \mathcal{T}^{3 \mid \mathcal{N}} \rightarrow \mathrm{GL}(n, \mathbb{C})$ which can be understood as an isomorphism $f_{+-}^{\tau}: \mathcal{E}_{-}^{\tau} \rightarrow \mathcal{E}_{+}^{\tau}$ between two trivial complex vector bundles $\mathcal{E}_{ \pm}^{\tau} \rightarrow \mathcal{T}^{3 \mid \mathcal{N}}$. We assume that $f_{+-}^{\tau}$ satisfies the reality condition

$$
\left(f_{+-}^{\tau}\left(z_{+}^{\alpha}, \lambda_{+}, \eta_{i}^{+}\right)\right)^{\dagger}=f_{+-}^{\tau}\left(z_{+}^{\alpha}, \lambda_{+}, \eta_{i}^{+}\right) .
$$

Given such a function $f_{+-}^{\tau}$, one can extend it holomorphically into a neighbourhood $\hat{\mathcal{U}}$ of $\mathcal{T}^{3 \mid \mathcal{N}}$ in $\mathcal{P}^{3 \mid \mathcal{N}}$, such that the extension $f_{+-}$of $f_{+-}^{\tau}$ satisfies the reality condition

$$
\left(f_{+-}\left(\tau_{1}\left(z_{+}^{\alpha}, \lambda_{+}, \eta_{i}^{+}\right)\right)\right)^{\dagger}=f_{+-}\left(z_{+}^{\alpha}, \lambda_{+}, \eta_{i}^{+}\right),
$$

generalizing equation (D.1). The function $f_{+-}$is holomorphic on $\hat{\mathcal{U}}=\hat{\mathcal{U}}_{+} \cap$ $\hat{\mathcal{U}}_{-}$and can be identified with a transition function of a holomorphic vector bundle $\mathcal{E}$ over $\mathcal{P}^{3 \mid \mathcal{N}}=\hat{\mathcal{U}}_{+} \cup \hat{\mathcal{U}}_{-}$which glues together two trivial bundles $\mathcal{E}_{+}=\hat{\mathcal{U}}_{+} \times \mathbb{C}^{n}$ and $\mathcal{E}_{-}=\hat{\mathcal{U}}_{-} \times \mathbb{C}^{n}$. Obviously, the two trivial vector bundles $\mathcal{E}_{ \pm}^{\tau} \rightarrow \mathcal{T}^{3 \mid \mathcal{N}}$ are restrictions of the trivial bundles $\mathcal{E}_{ \pm} \rightarrow \hat{\mathcal{U}}_{ \pm}$to $\mathcal{T}^{3 \mid \mathcal{N}}$

In the twistor approach, it is assumed that the bundle $\mathcal{E}$ is holomorphically trivial when restricted to any curve $\mathbb{C} P_{x_{\mathrm{R}}, \eta}^{1} \hookrightarrow \mathcal{P}^{3 \mid \mathcal{N}}$ and therefore there exists a gauge in which the restriction of the transition function $f_{+-}$to any $\mathbb{C} P_{x_{\mathrm{R}}, \eta}^{1}$ splits,

$$
f_{+-}=\psi_{+}^{-1} \psi_{-},
$$

into regular ${ }^{56}$ matrix-valued functions $\psi_{+}$and $\psi_{-}$defined on $\hat{\mathcal{U}}_{+}=\mathcal{P}_{+}^{3 \mid \mathcal{N}} \cup \hat{\mathcal{U}}$ and $\hat{\mathcal{U}}_{-}=\mathcal{P}_{-}^{3 \mid \mathcal{N}} \cup \hat{\mathcal{U}}$ and holomorphic in $\lambda_{+} \in H_{+}^{2}$ and $\lambda_{-} \in H_{-}^{2}$, respectively. Note that the condition (D.2) is satisfied if

$$
\psi_{+}^{-1}\left(\tau_{1}\left(x_{\mathrm{R}}^{\alpha \dot{\alpha}}, \lambda_{+}, \eta_{i}^{\dot{\alpha}}\right)\right)=\psi_{-}^{\dagger}\left(x_{\mathrm{R}}^{\alpha \dot{\alpha}}, \lambda_{-}, \eta_{i}^{\dot{\alpha}}\right) .
$$

Restricting (D.3) to $S_{x_{\mathrm{R}}, \eta}^{1} \hookrightarrow \mathbb{C} P_{x_{\mathrm{R}}, \eta}^{1}$, we obtain

$$
f_{+-}^{\tau}=\left(\psi_{+}^{\tau}\right)^{-1} \psi_{-}^{\tau} \text { with }\left(\psi_{+}^{\tau}\right)^{-1}=\left(\psi_{-}^{\tau}\right)^{\dagger},
$$

\footnotetext{
${ }^{56}$ Recall that by "regular," we mean smooth with nonvanishing determinant.
} 
where $\psi_{ \pm}^{\tau}$ are restrictions to $\mathcal{R}_{\mathrm{R}}^{4 \mid 2 \mathcal{N}} \times S^{1}$ of the matrix-valued functions $\psi_{ \pm}$ given by (D.3) and (D.4). Thus, the initial twistor data consist of a realanalytic function ${ }^{57} f_{+-}^{\tau}$ on $\mathcal{T}^{3 \mid \mathcal{N}}$ satisfying (D.1) together with a splitting (D.5), from which we construct a holomorphic vector bundle $\mathcal{E}$ over $\mathcal{P}^{3 \mid \mathcal{N}}$ with a transition function $f_{+-}$which is a holomorphic extension of $f_{+-}^{\tau}$ to $\hat{\mathcal{U}} \supset \mathcal{T}^{3 \mid \mathcal{N}}$. In other words, the space of real twistor data is the moduli space of holomorphic vector bundles $\mathcal{E} \rightarrow \mathcal{P}^{3 \mid \mathcal{N}}$ with transition functions satisfying the reality conditions (D.2).

In the purely real setting, one considers a real-analytic $\mathrm{GL}(n, \mathbb{C})$-valued function $f_{+-}^{\tau}$ on $\mathcal{T}^{3 \mid \mathcal{N}}$ satisfying the hermiticity condition (D.1) and the real double fibration (C.5). Since the pull-back of $f_{+-}^{\tau}$ to $\mathcal{R}_{\mathrm{R}}^{4 \mid 2 \mathcal{N}} \times S^{1}$ has to be constant along the fibres of $\pi_{2}$, we obtain the constraint equations

$$
v_{\alpha}^{+} f_{+-}^{\tau}=0=v_{+}^{i} f_{+-}^{\tau},
$$

or equivalently

$$
v_{\alpha}^{-} f_{+-}^{\tau}=0=v_{-}^{i} f_{+-}^{\tau},
$$

with the vector fields $v_{\alpha}^{ \pm}$and $v_{ \pm}^{i}$ from (C.6) and (C.8). Using the splitting (D.5) of $f_{+-}^{\tau}$ on fibres $S_{x_{\mathrm{R}}, \eta}^{1}$ of the projection $\pi_{1}$ in (C.5) and substituting $f_{+-}^{\tau}=\left(\psi_{+}^{\tau}\right)^{-1} \psi_{-}^{\tau}$ into (D.6), we obtain the linear systems (cf. (5.11)-(5.13))

$$
\begin{array}{ll}
\left(v_{\alpha}^{+}+\mathcal{A}_{\alpha}^{+}\right) \psi_{+}^{\tau}=0, & \left(v_{\alpha}^{-}+\mathcal{A}_{\alpha}^{-}\right) \psi_{-}^{\tau}=0, \\
\left(v_{+}^{i}+\mathcal{A}_{+}^{i}\right) \psi_{+}^{\tau}=0, & \left(v_{-}^{i}+\mathcal{A}_{-}^{i}\right) \psi_{-}^{\tau}=0 .
\end{array}
$$

Here $\mathcal{A}_{ \pm}=\left(\mathcal{A}_{\alpha}^{ \pm}, \mathcal{A}_{ \pm}^{i}\right)$ are relative connections on the bundles $\pi_{2}^{*} \mathcal{E}_{ \pm}^{\tau}$. From (D.8), one can find $\psi_{ \pm}^{\tau}$ for any given $\mathcal{A}_{\alpha}^{ \pm}$and $\mathcal{A}_{ \pm}^{i}$ and vice versa, i.e., find $\mathcal{A}_{\alpha}^{ \pm}$and $\mathcal{A}_{ \pm}^{i}$ for given $\psi_{ \pm}^{\tau}$ by the formulæ

$$
\begin{array}{ll}
\mathcal{A}_{\alpha}^{+}=\psi_{+}^{\tau} v_{\alpha}^{+}\left(\psi_{+}^{\tau}\right)^{-1}=\psi_{-}^{\tau} v_{\alpha}^{+}\left(\psi_{-}^{\tau}\right)^{-1}, & \mathcal{A}_{\alpha}^{-}=\psi_{+}^{\tau} v_{\alpha}^{-}\left(\psi_{+}^{\tau}\right)^{-1}=\psi_{-}^{\tau} v_{\alpha}^{-}\left(\psi_{-}^{\tau}\right)^{-1}, \\
\mathcal{A}_{+}^{i}=\psi_{+}^{\tau} v_{+}^{i}\left(\psi_{+}^{\tau}\right)^{-1}=\psi_{-}^{\tau} v_{+}^{i}\left(\psi_{-}^{\tau}\right)^{-1}, & \mathcal{A}_{-}^{i}=\psi_{+}^{\tau} v_{-}^{i}\left(\psi_{+}^{\tau}\right)^{-1}=\psi_{-}^{\tau} v_{-}^{i}\left(\psi_{-}^{\tau}\right)^{-1} .
\end{array}
$$

The compatibility conditions of the linear systems (D.8) read

$$
\begin{aligned}
v_{\alpha}^{ \pm} \mathcal{A}_{\beta}^{ \pm}-v_{\beta}^{ \pm} \mathcal{A}_{\alpha}^{ \pm}+\left[\mathcal{A}_{\alpha}^{ \pm}, \mathcal{A}_{\beta}^{ \pm}\right]=0, \\
v_{\alpha}^{ \pm} \mathcal{A}_{ \pm}^{i}-v_{ \pm}^{i} \mathcal{A}_{\alpha}^{ \pm}+\left[\mathcal{A}_{\alpha}^{ \pm}, \mathcal{A}_{ \pm}^{i}\right]=0, \\
v_{ \pm}^{i} \mathcal{A}_{ \pm}^{j}-v_{ \pm}^{j} \mathcal{A}_{ \pm}^{i}+\left[\mathcal{A}_{ \pm}^{i}, \mathcal{A}_{ \pm}^{j}\right]=0 .
\end{aligned}
$$

\footnotetext{
${ }^{57}$ One could also consider the extension $f_{+-}$and the splitting (D.5) even if $f_{+-}^{\tau}$ is not analytic, but in this case, the solutions to the super-SDYM equations can be singular. Such solutions are not related with holomorphic bundles.
} 
Geometrically, these equations imply flatness of the curvature of the relative connections $\mathcal{A}_{ \pm}=\left(\mathcal{A}_{\alpha}^{ \pm}, \mathcal{A}_{ \pm}^{i}\right)$ on the bundles $\pi_{2}^{*} \mathcal{E}_{ \pm}^{\tau}$ defined along the real $(2 \mid \mathcal{N})$-dimensional fibres of the projection $\pi_{2}$ in (C.5).

Recall that $\psi_{+}^{\tau}$ and $\psi_{-}^{\tau}$ extend holomorphically in $\lambda_{+}$and $\lambda_{-}$to $H_{+}^{2}$ and $H_{-}^{2}$, respectively, and therefore we obtain from (D.9) that $\mathcal{A}_{\alpha}^{ \pm}=\lambda_{ \pm}^{\dot{\alpha}} \mathcal{A}_{\alpha \dot{\alpha}}$ and $\mathcal{A}_{ \pm}^{i}=\lambda_{ \pm}^{\dot{\alpha}} \mathcal{A}_{\dot{\alpha}}^{i}$, where $\mathcal{A}_{\alpha \dot{\alpha}}$ and $\mathcal{A}_{\dot{\alpha}}^{i}$ do not depend on $\lambda_{ \pm}$. Then the compatibility conditions (D.10) of the linear systems (D.8) reduce to equations (5.14). In Section 5, it was demonstrated that for $\varepsilon=+1$, these equations are equivalent to the field equations of $\mathcal{N}$-extended SDYM theory on $\mathbb{R}^{2,2}$. Thus, there are bijections between the moduli spaces of solutions to equations (D.10), the field equations of $\mathcal{N}$-extended super SDYM theory on $\mathbb{R}^{2,2}$ and the moduli space of $\tau_{1}$-real holomorphic vector bundles $\mathcal{E}$ over $\mathcal{P}^{3 \mid \mathcal{N}}$.

Consider now the extension of the linear systems (D.8) to open domains $\hat{\mathcal{U}}_{ \pm}=\mathcal{P}_{ \pm}^{3 \mid \mathcal{N}} \cup \hat{\mathcal{U}} \supset \mathcal{T}^{3 \mid \mathcal{N}}$

$$
\begin{aligned}
\left(\bar{V}_{\alpha}^{ \pm}+\mathcal{A}_{\alpha}^{ \pm}\right) \psi_{ \pm} & =0, \\
\left(\bar{\partial}_{ \pm}^{i}+\mathcal{A}_{ \pm}^{i}\right) \psi_{ \pm} & =0, \\
\partial_{\bar{\lambda}_{ \pm}} \psi_{ \pm} & =0,
\end{aligned}
$$

where $\bar{V}_{\alpha}^{ \pm}$and $\bar{\partial}_{ \pm}^{i}$ are vector fields of type $(0,1)$ on $\hat{\mathcal{U}}_{ \pm}^{s}:=\hat{\mathcal{U}}_{ \pm} \backslash\left(\mathcal{P}_{0, \mathcal{N}}\right)$ as given in (4.19) and (4.20). These vector fields annihilate $f_{+-}$and from this fact and the splitting (D.3), one can also derive equations (D.11). Recall that due to the existence of a diffeomorphism between the spaces $\mathcal{R}_{\mathrm{R}}^{4 \mid 2 \mathcal{N}} \times H^{2}$ and $\tilde{\mathcal{P}}^{3 \mid \mathcal{N}}$ which is described in (C.10) and (C.11), the double fibration (C.9) simplifies to the nonholomorphic fibration (C.12). Moreover, since the restrictions of the bundle $\mathcal{E} \rightarrow \mathcal{P}^{3 \mid \mathcal{N}}$ to the fibres of the fibration (4.3) are trivial, there exist regular matrix-valued functions $\hat{\psi}_{ \pm}$on $\hat{\mathcal{U}}_{ \pm}^{s}$ such that

$$
f_{+-}=\hat{\psi}_{+}^{-1} \hat{\psi}_{-}
$$

on $\hat{\mathcal{U}}^{s}=\hat{\mathcal{U}} \backslash\left(\mathcal{P}_{0, \mathcal{N}}\right)$ and

$$
\bar{\partial}_{ \pm}^{i} \hat{\psi}_{ \pm}=0
$$

The existence of this gauge was already implied in [2]. Additionally, we impose the reality condition

$$
\hat{\psi}_{+}^{-1}\left(x_{\mathrm{R}}^{\alpha \dot{\alpha}}, \frac{1}{\bar{\lambda}_{+}}, \frac{\bar{\eta}_{i}^{+}}{\bar{\lambda}_{+}}\right)=\hat{\psi}_{-}^{\dagger}\left(x_{\mathrm{R}}^{\alpha \dot{\alpha}}, \lambda_{-}, \eta_{i}^{-}\right)
$$

on $\hat{\psi}_{ \pm}$. Although $\hat{\mathcal{U}}^{s}$ consists of two disconnected pieces, the functions $\hat{\psi}_{ \pm}$ are not independent on each piece because of the condition (D.14), which 
also guarantees (D.2) on $\hat{\mathcal{U}}^{s}$. The functions $\hat{\psi}_{ \pm}$and their inverses are illdefined on $\mathcal{P}_{0, \mathcal{N}}$ since the restriction of $\pi_{2}$ to $\mathcal{R}_{\mathrm{R}}^{4 \mid 2 \mathcal{N}} \times S^{1}$ is a noninvertible projection onto $\mathcal{T}^{3 \mid \mathcal{N}}$, see (C.10). Equating (D.2) and (D.12), one sees that the singularities of $\hat{\psi}_{ \pm}$on $\mathcal{P}_{0, \mathcal{N}}$ split off in a matrix-valued function $\varphi^{-1}$, i.e.,

$$
\hat{\psi}_{ \pm}=\varphi^{-1} \psi_{ \pm}
$$

which disappears from

$$
f_{+-}=\hat{\psi}_{+}^{-1} \hat{\psi}_{-}=\left(\psi_{+}^{-1} \varphi\right)\left(\varphi^{-1} \psi_{-}\right)=\psi_{+}^{-1} \psi_{-} .
$$

Therefore, $f_{+-}$is a nonsingular holomorphic matrix-valued function on all of $\hat{\mathcal{U}}$.

From (D.12)-(D.16) it follows that on $\tilde{\mathcal{P}}^{3 \mid \mathcal{N}}$, we have a well-defined gauge transformation (5.24)-(5.27) generated by $\varphi$ and one can introduce gauge potentials $\hat{\mathcal{A}}_{+}^{0,1}$ and $\hat{\mathcal{A}}_{-}^{0,1}$ which are defined on $\hat{\mathcal{U}}_{+}^{s}$ and $\hat{\mathcal{U}}_{-}^{s}$, respectively, but not on $\mathcal{P}_{0, \mathcal{N}}$. By construction, $\hat{\mathcal{A}}^{0,1}=\left(\hat{\mathcal{A}}_{+}^{0,1}, \hat{\mathcal{A}}_{-}^{0,1}\right)$ satisfies the hCS equations (5.31) and (5.32) on $\tilde{\mathcal{P}}^{3 \mid \mathcal{N}}=\mathcal{P}_{+}^{3 \mid \mathcal{N}} \cup \mathcal{P}_{-}^{3 \mid \mathcal{N}}$ which are equivalent to the $\mathcal{N}$-extended super-SDYM equations on $\mathbb{R}^{2,2}$. Conversely, having a solution $\hat{\mathcal{A}}^{0,1}$ of the hCS field equations on the space $\tilde{\mathcal{P}}^{3 \mid \mathcal{N}}$, one can find regular matrix-valued functions $\hat{\psi}_{+}$on $\hat{\mathcal{U}}_{+}^{s}$ and $\hat{\psi}_{-}$on $\hat{\mathcal{U}}_{-}^{s}$ which satisfy the reality condition (D.14). These functions define a further function $f_{+-}^{s}=$ $\hat{\psi}_{+}^{-1} \hat{\psi}_{-}: \hat{\mathcal{U}}^{s} \rightarrow \operatorname{GL}(n, \mathbb{C})$ which can be completed to a holomorphic function $f_{+-}: \hat{\mathcal{U}} \rightarrow \operatorname{GL}(n, \mathbb{C})$ due to (D.16). The latter one can be identified with a transition function of a holomorphic vector bundle $\mathcal{E}$ over the supertwistor space $\mathcal{P}^{3 \mid \mathcal{N}}$. The restriction of $f_{+-}$to $\mathcal{T}^{3 \mid \mathcal{N}}$ is a real-analytic function $f_{+-}^{\tau}$ which is not constrained by any differential equation. Thus, in the case $\varepsilon=+1$ (and also for the real structure $\tau_{0}$ ), one can either consider two trivial complex vector bundles $\mathcal{E}_{ \pm}^{\tau}$ defined over the space $\mathcal{T}^{3 \mid \mathcal{N}}$ together with an isomorphism $f_{+-}^{\tau}: \mathcal{E}_{-}^{\tau} \rightarrow \mathcal{E}_{+}^{\tau}$ or a single complex vector bundle $\mathcal{E}$ over the space $\mathcal{P}^{3 \mid \mathcal{N}}$. However, the appropriate hCS theory which has the same moduli space as the moduli space of (equivalence classes of) these bundles is defined on $\tilde{\mathcal{P}}^{3 \mid \mathcal{N}}$. Moreover, real Chern-Simons theory on $\mathcal{T}^{3 \mid \mathcal{N}}$ has no moduli, since its solutions correspond to flat bundles over $\mathcal{T}^{3 \mid \mathcal{N}}$ with constant transition functions ${ }^{58}$ defined on the intersections of appropriate patches covering $\mathcal{T}^{3 \mid \mathcal{N}}$.

To sum up, there is a bijection between the moduli spaces of solutions to equations (D.10) and to the hCS field equations on the space $\tilde{\mathcal{P}}^{3 \mid \mathcal{N}}$ since both moduli spaces are bijective to the moduli space of holomorphic vector

\footnotetext{
${ }^{58}$ Note that these transition functions are in no way related to the transition functions $f_{+-}$of the bundles $\mathcal{E}$ over $\mathcal{P}^{3 \mid \mathcal{N}}$ or to the functions $f_{+-}^{\tau}$ defined on the whole of $\mathcal{T}^{3 \mid \mathcal{N}}$.
} 
bundles over $\mathcal{P}^{3 \mid \mathcal{N}}$. In fact, whether one uses the real supertwistor space $\mathcal{T}^{3 \mid \mathcal{N}}$, or works with its complexification $\mathcal{P}^{3 \mid \mathcal{N}}$, is partly a matter of taste. However, the complex approach is more geometrical and more natural from the point of view of an action principle and the topological B-model. For example, equations (D.10) cannot be transformed by a gauge transformation to a set of differential equations on $\mathcal{T}^{3 \mid \mathcal{N}}$ as it was possible on $\tilde{\mathcal{P}}^{3 \mid \mathcal{N}}$ in the complex case. This is due to the fact that the transition function $f_{+-}$, which was used as a link between the two sets of equations in the complex case does not satisfy any differential equation after restriction to $\mathcal{T}^{3 \mid \mathcal{N}}$. From this we see that we cannot expect any action principle on $\mathcal{T}^{3 \mid \mathcal{N}}$ to yield equations equivalent to (D.10) as we had in the complex case. For these reasons, we have chosen to use the complex approach throughout the paper.

\section{References}

[1] E. Witten, Chern-Simons gauge theory as a string theory, Prog. Math. 133 (1995), 637 [hep-th/9207094].

[2] E. Witten, Perturbative gauge theory as a string theory in twistor space, Commun. Math. Phys. 252 (2004), 189 [hep-th/0312171].

[3] N. Berkovits, An alternative string theory in twistor space for $N=4$ super-Yang-Mills, Phys. Rev. Lett. 93 (2004) 011601 [hep-th/0402045].

[4] N. Berkovits, Self-dual super-Yang-Mills as a string theory in $(x, \theta)$ space, JHEP 0405 (2004), 034 [hep-th/0403280].

[5] A. Neitzke and C. Vafa, $N=2$ strings and the twistorial Calabi-Yau, hep-th/0402128.

[6] P.A. Grassi and P. van Nieuwenhuizen, Harmonic superspaces from superstrings, Phys. Lett. B 593 (2004), 271 [hep-th/0402189].

[7] N. Nekrasov, H. Ooguri and C. Vafa, S-duality and topological strings, JHEP 0410 (2004), 009 [hep-th/0403167].

[8] N. Berkovits and L. Motl, Cubic twistorial string field theory, JHEP 0404 (2004), 056 [hep-th/0403187].

[9] M. Aganagic and C. Vafa, Mirror symmetry and supermanifolds, hep-th/0403192.

[10] W. Siegel, Untwisting the twistor superstring, hep-th/0404255.

[11] V.P. Nair, A current algebra for some gauge theory amplitudes, Phys. Lett. B 214 (1988), 215.

[12] R. Roiban, M. Spradlin and A. Volovich, A googly amplitude from the $B$-model in twistor space, JHEP 0404 (2004), 012 [hep-th/0402016]; On the tree-level S-matrix of Yang-Mills theory, Phys. Rev. D 70 (2004), 
026009 [hep-th/0403190]; R. Roiban and A. Volovich, All googly amplitudes from the B-model in twistor space, Phys. Rev. Lett. 93 (2004), 131602 [hep-th/0402121].

[13] F. Cachazo, P. Svrcek and E. Witten, $M H V$ vertices and tree amplitudes in gauge theory, JHEP 0409 (2004), 006 [hep-th/0403047].

[14] C.J. Zhu, The googly amplitudes in gauge theory, JHEP 0404 (2004), 032 [hep-th/0403115].

[15] E. Witten, Parity invariance for strings in twistor space, Adv. Theor. Math. Phys. 8 (2004), 779 [hep-th/0403199].

[16] G. Georgiou and V.V. Khoze, Tree amplitudes in gauge theory as scalar MHV diagrams, JHEP 0405 (2004), 070 [hep-th/0404072].

[17] S. Gukov, L. Motl and A. Neitzke, Equivalence of twistor prescriptions for super Yang-Mills, hep-th/0404085.

[18] S. Giombi, R. Ricci, D. Robles-Llana and D. Trancanelli, A note on twistor gravity amplitudes, JHEP 0407 (2004), 059 [hep-th/0405086].

[19] R. Penrose, The twistor program, Rept. Math. Phys. 12 (1977), 65.

[20] R.S. Ward, On self-dual gauge fields, Phys. Lett. A 61 (1977), 81.

[21] M.F. Atiyah, N.J. Hitchin and I.M. Singer, Self-duality in fourdimensional Riemannian geometry, Proc. Roy. Soc. Lond. A 362 (1978), 425.

[22] R.O. Wells Jr., Complex manifolds and mathematical physics, Bull. Amer. Math. Soc. 1 (1979), 296.

[23] N.J. Hitchin, Linear field equations on self-dual spaces, Proc. Roy. Soc. Lond. A 370 (1980), 173.

[24] N.M.J. Woodhouse, Real methods in twistor theory, Class. Quant. Grav. 2 (1985), 257.

[25] A. Ferber, Supertwistors and conformal supersymmetry, Nucl. Phys. B 132 (1978), 55.

[26] E. Witten, An interpretation of classical Yang-Mills theory, Phys. Lett. B 77 (1978), 394.

[27] J. Isenberg, P.B. Yasskin and P.S. Green, Non-self-dual gauge fields, Phys. Lett. B 78 (1978), 462.

[28] G.M. Henkin and Y.I. Manin, Twistor description of classical YangMills-Dirac fields, Phys. Lett. B 95 (1980) 405; The Yang-Mills-Dirac equations as the Cauchy-Riemann equations in the twistor space, Yad. Fiz. 35 (1982), 1610.

[29] A.M. Semikhatov, Supersymmetric instanton, JETP Lett. 35 (1982), 560 . 
[30] I.V. Volovich, Super self-duality for supersymmetric Yang-Mills theory, Phys. Lett. B 123 (1983), 329; Supersymmetric Yang-Mills theories and twistors, Phys. Lett. B 129 (1983), 429; Supersymmetric YangMills equations as an inverse scattering problem, Lett. Math. Phys. 7 (1983), 517.

[31] J.P. Harnad, J. Hurtubise, M. Legaré and S. Shnider, Constraint equations and field equations in supersymmetric $N=3$ Yang-Mills theory, Nucl. Phys. B 256 (1985), 609; J.P. Harnad and S. Shnider, Constraints and field equations for ten-dimensional super Yang-Mills theory, Commun. Math. Phys. 106 (1986), 183.

[32] J. Tafel, The Riemann-Hilbert problem for the supersymmetric constraint equations, J. Math. Phys. 28 (1987), 240.

[33] J.P. Harnad, J. Hurtubise and S. Shnider, Supersymmetric Yang-Mills equations and supertwistors, Ann. Phys. 193 (1989), 40.

[34] G.M. Henkin and R.G. Novikov, The Yang-Mils fields, the RadonPenrose transform, and the Cauchy-Riemann equations. Encyclopaedia of Mathematical Sciences, 54, Springer-Verlag, Berlin, 1993 [Russian: VINITI, Moscow, 1989].

[35] W. Siegel, The $N=2$ (4) string theory is self-dual $N=4$ Yang-Mills theory, Phys. Rev. D 46 (1992), R3235 [hep-th/9205075].

[36] S.V. Ketov, H. Nishino and S.J. Gates Jr. Self-dual supersymmetry and supergravity in Atiyah-Ward space-time, Nucl. Phys. B 393 (1993), 149 [hep-th/9207042].

[37] C. Devchand and V. Ogievetsky, Super self-duality as analyticity in harmonic superspace, Phys. Lett. B 297 (1992), 93 [hep-th/9209120].

[38] P.S. Howe and G.G. Hartwell, A superspace survey, Class. Quant. Grav. 12 (1995), 1823.

[39] E. Sokatchev, An action for $N=4$ supersymmetric self-dual YangMills theory, Phys. Rev. D 53 (1996), 2062 [hep-th/9509099].

[40] C. Devchand and V. Ogievetsky, Interacting fields of arbitrary spin and $N>4$ supersymmetric self-dual Yang-Mills equations, Nucl. Phys. B 481 (1996), 188 [hep-th/9606027].

[41] A.D. Popov, Self-dual Yang-Mills: Symmetries and moduli space, Rev. Math. Phys. 11 (1999), 1091 [hep-th/9803183].

[42] A.D. Popov, Holomorphic Chern-Simons-Witten theory: from $2 D$ to $4 D$ conformal field theories, Nucl. Phys. B 550 (1999), 585 [hepth/9806239]; Holomorphic analogs of topological gauge theories, Phys. Lett. B 473 (2000), 65 [hep-th/9909135].

[43] T.A. Ivanova and A.D. Popov, Dressing symmetries of holomorphic BF theories, J. Math. Phys. 41 (2000), 2604 [hep-th/0002120]; 
Cech, Dolbeault and de Rham cohomologies in Chern-Simons and BF theories, Proc. of the Intern. Colloquium on Group Theoretical Methods in Physics, eds. A.N. Sissakian and G.S. Pogosian, 1, Dubna (2002), 138 [hep-th/0101150].

[44] R. Penrose and W. Rindler, Spinors and space-time, 1 Cambridge University Press, Cambridge, 1984; Spinors and space-time, 2 Cambridge University Press, Cambridge, 1985.

[45] Y.I. Manin, Gauge field theory and complex geometry, Springer, Berlin, 1988 [Russian: Nauka, Moscow, 1984].

[46] R.S. Ward and R.O. Wells, Twistor geometry and field theory, Cambridge University Press, Cambridge, 1990.

[47] L.J. Mason and N.M.J. Woodhouse, Integrability, self-duality, and twistor theory, Clarendon Press, Oxford, 1996.

[48] M. Kotrla and J. Niederle, Supertwistors and superspace, Czech. J. Phys. B 35 (1985), 602.

[49] J. Lukierski and W.J. Zakrzewski, Euclidean supersymmetrization of instantons and self-dual monopoles, Phys. Lett. B 189 (1987), 99.

[50] H. Ooguri and C. Vafa, Geometry of $N=2$ strings, Nucl. Phys. B 361 (1991), 469; Self-duality and $N=2$ string magic, Mod. Phys. Lett. A 5 (1990), 1389; All loop $N=2$ string amplitudes, Nucl. Phys. B 451 (1995), 121 [hep-th/9505183].

[51] N. Berkovits, Super Poincaré invariant superstring field theory, Nucl. Phys. B 450 (1995), 90 [Erratum-ibid. B 459 (1996), 439] [hepth/9503099]; N. Berkovits and W. Siegel, Covariant field theory for self-dual strings, Nucl. Phys. B 505 (1997), 139 [hep-th/9703154].

[52] N. Berkovits and C. Vafa, $N=4$ topological strings, Nucl. Phys. B 433 (1995), 123 [hep-th/9407190].

[53] O. Lechtenfeld and A.D. Popov, On the integrability of covariant field theory for open $N=2$ strings, Phys. Lett. B 494 (2000), 148 [hepth/0009144]; O. Lechtenfeld, A.D. Popov and S. Uhlmann, Exact solutions of Berkovits' string field theory, Nucl. Phys. B 637 (2002), 119 [hep-th/0204155].

[54] A. Kling, O. Lechtenfeld, A.D. Popov and S. Uhlmann, On nonperturbative solutions of superstring field theory, Phys. Lett. B $\mathbf{5 5 1}$ (2003), 193 [hep-th/0209186]; Solving string field equations: new uses for old tools, Fortsch. Phys. 51 (2003), 775 [hep-th/0212335]; A. Kling and S. Uhlmann, String field theory vertices for fermions of integral weight, JHEP 0307 (2003), 061 [hep-th/0306254]; M. Ihl, A. Kling and S. Uhlmann, String field theory projectors for fermions of integral weight, JHEP 0403 (2004), 002 [hep-th/0312314]. 
[55] T.A. Ivanova and O. Lechtenfeld, Hidden symmetries of the open $N=2$ string, Int. J. Mod. Phys. A 16 (2001), 303 [hep-th/0007049].

[56] M. Ihl and S. Uhlmann, Noncommutative extended waves and solitonlike configurations in $N=2$ string theory, Int. J. Mod. Phys. A 18 (2003), 4889 [hep-th/0211263].

[57] Z. Khviengia, H. Lu, C.N. Pope, E. Sezgin, X.J. Wang and K.W. Xu, $N=1$ superstring in (2+2)-dimensions, Nucl. Phys. B 444 (1995), 468 [hep-th/9504121]; H. Lu, C.N. Pope and E. Sezgin, A search for new (2, 2) strings, Class. Quant. Grav. 12 (1995), 1913 [hep-th/9504122].

[58] S. Bellucci, A. Galajinsky and O. Lechtenfeld, A heterotic $N=2$ string with space-time supersymmetry, Nucl. Phys. B 609 (2001) 410 [hepth/0103049].

[59] P. Cartier, C. DeWitt-Morette, M. Ihl and C. Saemann, Supermanifolds-Application to supersymmetry, in Multiple facets of quantization and supersymmetry: Michael Marinov memorial volume, eds. M. Olshanetsky and A. Vainshtein, World Scientific, (2002), 412 [math-ph/0202026]. 